\title{
An Equilibrium Model of Term Structures of Bonds and Equities
}

\author{
Hideyuki Takamizawa * \\ Graduate School of Business Administration, \\ Hitotsubashi University \\ Kunitachi Tokyo 186-8601, JAPAN \\ E-mail: Hi.Takamizawa@r.hit-u.ac.jp
}

First draft: March 15, 2017

This draft: July 1, 2018

\begin{abstract}
This study proposes an equilibrium model of term structures of bonds and equities, which has a similar descriptive ability to a reduced-form model proposed by Lettau and Wachter (LW) (J. Financial Economics, 2011), and yet offers economic implications about preferences and consumption dynamics. The ability is obtained by letting parameters of recursive utility depend on state variables of the economy. The model is calibrated by matching it with the LW model, showing that it can produce the term structure of real interest rates with either a positive or negative slope and the term structure of dividend risk premiums with a negative slope, both of which stand as challenges to any pricing models. It also shows that while an implied behavior of state-dependent time preference is reasonable, modifications of parameter values and cash flow processes are necessary for state-dependent risk aversion to behave reasonably.
\end{abstract}

Keywords: Term structure, Interest rate, Dividend strip, Risk premium, Sharpe ratio, Recursive utility, State-dependent preference.

${ }^{*}$ I started this research while I was visiting at Northwestern Kellogg. I am grateful to Torben Andersen for his great hospitality. I would like to thank Yoshio Nozawa and Masataka Suzuki for helpful comments and Toru Igarashi for research assistance. Financial support from JSPS KAKENHI (Grant Number 15K03538), ISHII Memorial Securities Research Promotion Foundation, and Zengin Foundation for Studies on Economics and Finance are gratefully acknowledged. 


\section{Introduction}

The pricing of cash flows at various points in time is one of central issues in finance. The term structure of interest rates, which has long been studied, is based on fixed cash flows. Stochastic cash flows such as dividends lead to the term structure of dividend strips or zero-coupon equities, which is of relatively recent focus. The purpose of this study is to build an equilibrium model of term structures of zero-coupon bonds and equities and discuss preferences and consumption dynamics implicit in these term structures.

Essentially, any equilibrium models can price any assets. However, there are not many such models aimed at explaining both zero-coupon bonds and equities from a term-structure perspective. In modeling the term structure, at least two challenges are known. The first is the term structure of real interest rates that is either upward-, flat-, or downward-sloping. This indecisive shape in turn requires model's flexibility. The second is the term structure of risk premiums of dividend strips. It is on average downward-sloping, which is evidenced from various data sources: index options (van Binsbergen, Brandt, and Koijen, 2012); index dividend futures (van Binsbergen et al, 2013; van Binsbergen and Koijen, 2017); and the cross-section of stocks (Weber, 2017). ${ }^{1}$ However, as illustrated by van Binsbergen et al. (2012), the downward slope is difficult to explain using well-established equilibrium models, such as the external habit model of Campbell and Cochrane (1999), the long-run risks model of Bansal and Yaron (2004), and the disaster model of Barro (2009), and Gabaix (2012). These challenges motivate recent studies to improve equilibrium models, which are reviewed below in comparison with ours.

Without imposing equilibrium conditions, it may not be very difficult to model the term structures of real interest rates and dividend risk premiums consistently with stylized facts. Indeed, Lettau and Wachter (LW) (2011) propose a reduced-form model that can explain these term structures. The key to their success is the specification of the stochastic discount factor (SDF), which increases one for one with a negative shock to realized dividend growth. This specification is effective for raising the risk of holding short-term dividend strips. Furthermore, LW assume that a negative shock to realized dividend growth is highly likely to increase expected dividend growth. This assumption makes long-term dividend strips less risky because they go up in value when the SDF is high. Consequently, the term structure of dividend risk premiums is sloped downward. In addition, the LW model can generate either an upward-, flat-, or downward-

\footnotetext{
${ }^{1}$ See also Schulz (2016), pointing out that the evidence for the downward-slope is not decisive when returns to short-term dividend claims are adjusted for taxies or liquidities.
} 
sloping term structure of real interest rates by simply controlling for the correlation between realized dividend growth and real risk-free rate.

These important mechanisms of the LW model are exogenous. Our goal is to endogenize them. The purpose of this study is to develop an equilibrium model that offers implications about preferences and consumption dynamics and yet has a similar descriptive ability to the reduced-form model proposed by LW. For this purpose, we ask what kind of utility function supports the LW's SDF. Our answer is to let parameters of a recursive utility function of the representative agent depend on state variables of the economy. Meanwhile, we model cash flow processes as simply as in the original LW model: we later consider a minimal extension of cash flows.

The idea of state-dependent preferences itself is not new. In fact, Gordon and St-Amour (2000, 2004), Melino and Yang (2003), Chabi-Yo, Garcia, and Renault (2008), Berrada, Detemple, and Rindisbacher (2013), and Dew-Becker (2014) consider models in which preference parameters themselves change over time: A more detailed review is provided below. A distinct feature of the current model is that both risk aversion parameter and time preference parameter are driven by many state variables such as (expected) consumption and inflation growth and financial variables such as risk-free rate and price of risks, so that the agent can fine-tune her preferences by looking at the economy and asset markets.

A state-dependent risk aversion is beneficial for amplifying the variation in the SDF and hence capturing high equity risk premiums. Besides, it leads to time-varying price of risks, which naturally explains time-varying risk premium (the product of the price of risks and an asset-specific quantity of risks) with the source of variation not limited to stochastic volatility, or stochastic intensity in the case of jumps, of cash flow processes. Furthermore, it offers an additional channel of raising the slope of the term structure of nominal interest rates other than the standard channel of a negative correlation between consumption and inflation growth. A state-dependent time preference also has an advantage of generating various shapes in the term structure of real interest rates. Suppose, for example, there is a shock that increases the SDF. If this shock also affects the agent's time-preference in a way where she more heavily discounts future cash flows, the prices of real bonds will fall with the fall more significant for longerterm bonds due to the compound effect. The real bonds therefore cannot be hedge against events of raising the SDF, and the real term structure will be upward-sloping. This mechanism might also be useful when introduced into the LRR model of Bansal and Yaron (2004). One 
of the criticisms to the LRR model is that it cannot generate a flat- or upward-sloping real term structure when calibrated consistently with various moments of financial markets because then, real bonds work as hedge against long-run risks about consumption growth; see Bansal, Kiku, and Yaron (2012), and Beeler and Campbell (2012) for debate over the LRR model. State-dependent time preference has potential of relaxing this restriction of the LRR model.

The parameters of the proposed model are calibrated by matching it with the LW model. This calibration method has two advantages. The first is to achieve a similar descriptive ability to the LW model. Indeed, the proposed model can closely replicate various term structure shapes generated by the LW model. The second is to obtain an equilibrium foundation of the LW model. Indeed, it is possible to imply preferences and consumption dynamics from the LW model through the calibration of the proposed model. The calibration results, however, contain unrealistic implications about preferences and/or consumption dynamics. Most notably, given consumption volatility of less than $4 \%$ per year, the mean and standard deviation of a statedependent risk aversion reach 150 and 128, respectively. When the mean risk aversion is reduced to 30 , then an implied consumption volatility is nearly $9 \%$.

One possibility of these unrealistic economic implications is that parameter values originally calibrated by LW (2011) are not appropriate. Originally, the LW model is a reduced-form model so that there are many combinations of parameter values that can explain observed term structures. However, once some equilibrium conditions are imposed, there are not many combinations of parameter values that are consistent with not only observed term structures but realistic preferences and consumption dynamics. Our model has an advantage of revealing which combinations are more appropriate. Another possibility is that the dynamics of cash flows in the LW model are too simple. We then slightly deviate from the LW model by introducing jumps into consumption and dividend growth, which are interpreted as disasters. The change of parameter values and the modification of cash flow processes together are shown to be effective for making the behavior of both risk aversion and consumption growth economically plausible while keeping the ability to explain various term structures.

\section{Related literature}

Our model extends the recursive utility function of Epstein and Zin (1989, 1991), and Weil (1989) in a way where the parameters of risk aversion and time preference depend on state variables of the economy. Melino and Yang (2003) consider the recursive utility function with 
state-dependent parameters more generally in that the elasticity of intertemporal substitution is also state-dependent; however, they do not model how these parameters evolve over time. The low of motion of the risk aversion is modeled by Gordon and St-Amour (2000), and ChabiYo, Garcia, and Renault (2008) using Markov switching processes. Berrada, Detemple, and Rindisbacher (2013) also use them for modeling the dynamics of both risk aversion and time preference. Gordon and St-Amour (2004), and Dew-Becker (2014) model the risk aversion as driven by auto-regressive processes. Our model is similar to the last two studies regarding how to model time variation but different from them in that time preference is also state-dependent and that the preference parameters are driven by many state variables driving the economy.

Compared with state-dependent risk aversion, state-dependent time preference has been less of a focus. But Frederick, Loewenstein and O'Donoghue (2002) document that prior to the Samuelson's introduction of constant subjective discount rate, time-preference was considered to be not necessarily constant but rather affected by various factors including psychological ones. Halevy (2015) conducts a field experiment about time preference and shows that some subjects (classroom students in his study) exhibit time-varying rate of subjective discount. The direct modeling of time preference as a function of state variables - an approach taken by this study - is different from the well-established, horizon-dependent time preference that discounts nearby cash flows more heavily than distant ones; see, for example, Thaler (1981). However, Harris and Laibson (2001), and Luttmer and Mariotti (2003) show that the horizon-dependent time preference leads to an effective rate of subjective discount that depends on state variables affecting endowment growth (unless the agent has log utility). As such, it may not be unreasonable to consider state-dependent time preference. Besides, from an asset pricing perspective, it is beneficial for generating the term structure of real interest rates, the average shape of which is indecisive.

Recent studies develop equilibrium asset pricing models aimed at explaining a downwardsloping term structure of dividend risk premiums. We limit our attention to some of these studies that explicitly present the results for the entire term structures of risk premiums and return volatilities of dividend strips, which are summarized in Table 1: A broader review is provided by van Binsbergen and Koijen (2017).

There are mainly two approaches for improving equilibrium models: One is to improve preferences or discounts as taken by this study and the other cash flows. The recent studies taking the preference approach are as follows. Curatola (2015) considers heterogeneous agents 
who have loss-averse utility, where the reference point between gain and loss is set at the level of external consumption habit, so that unlike many standard habit formation models, consumption is allowed to be below the habit. Because the loss-averse agents are willing to hold long-term dividend claims to hedge risks of future consumption being below the habit, the term structure of dividend risk premiums is downward-sloping. The term structure of real interest rates, on the other hand, is upward-sloping because long-term real bonds cannot be hedge against increase in habit and hence decrease in surplus consumption. Doh and Wu (2016) impose a structure on the LRR model such that both the equilibrium wealth-consumption ratio and the price of a one-period dividend strip are quadratic functions of state variables and then reverse-engineer consumption and dividend processes consistent with the imposed structure. The resulting risk premiums of dividend strips are first decreasing with maturity and then increasing, which is not surprising as the premiums are also quadratic in state variables.

The recent studies that modify cash flow processes propose various mechanisms that make short-run growth of dividends volatile and pro-cyclical relative to long-run growth. Belo, CollinDufresne, and Goldstein (2015) consider as a mechanism a stationary financial leverage ratio. In their model, in response to temporal increase (decrease) in corporate earnings, measured by EBIT, a firm is assumed to increase (decrease) debt to keep the leverage ratio to a stationary level, which further increases (decreases) cash distributed to shareholders as dividends. Consequently, dividends change more intensely than earnings in the short-run. In the long-run, however, both EBIT and dividends are exposed to the same amount of risks due to the stationarity of the leverage ratio that makes EBIT and dividend cointegrated. Favilukis and Lin (2016) consider as a mechanism wage rigidity in a production economy, where a negative transitory shock to technology, corresponding to bad states of the economy, reduces dividends more than wages that are settled infrequently. Lopez, Lopez-Salido and Vazquez-Grande (2015) consider a similar logic but instead use nominal rigidity that induces a countercyclical wage share of output and hence a procyclical dividend share. Marfè (2017) also uses the wage channel together with limited participation of asset markets. Specifically, in his model, shareholders who receive and consume dividends provide wage insurance to workers, which is effective for the short-run but not for the long-run because both dividends and wages are co-integrated so that they share the same long-run risks. Then, dividends, or equivalently shareholders' consumption in equilibrium, are more prone to transitory shocks than wages. Meanwhile, only shareholders can access to asset markets. Consequently, from the eyes of the pricing agents (i.e., shareholders), short-term 
dividend strips look riskier than long-term counterparts.

The above studies of the cash-flow approach seem to place more emphasis on short-run growth of dividends than long-run growth. Hasler and Marfè (2016) pay attention to both short-run and long-run growth. They introduce recovery after disaster into cash flow processes as well as stochastic mean of cash flow growth and stochastic intensity of disaster occurrence. While the latter two features alone generate an upward-sloping term structure of dividend risk premiums as does the Wachter (2013) model, fast recovery of dividend growth after a large negative shock reduces risks of holding long-term dividend strips, more than offsetting long-run risks associated with stochastic mean growth and disaster intensity.

A novel approach taken by Croce, Lettau, and Ludvigson (2015) for generating a downwardsloping term structure of dividend risk premiums is that they do not change cash flow processes from those originally specified by Bansal and Yaron (2004) but do change the way they are estimated. In their framework, the agent overestimates the impact of short-run shocks to consumption growth on dividend growth because she erroneously revises a long-run component of dividend growth that is irrelevant to short-run shocks to consumption growth. Consequently, she requires high premiums of holding short-term dividend strips. Conversely, long-run shocks to consumption growth, which are originally small, are difficult to infer from dividend growth because they are contaminated by large idiosyncratic shocks to dividend growth. Then, longrun consumption risks are not properly priced into long-term dividend strips, and they do not command large risk premiums.

The strength of the proposed model is flexibility. Indeed, it is as flexible as the LW model in term of generating various term structures despite the fact that equilibrium conditions are imposed. Extensibility is another strength. Since the proposed model modifies the preference of discount, it can easily be combined with more sophisticated cash-flow models.

The rest of the manuscript is organized as follows. Section 2 presents a model. Section 3 explains how to calibrate the parameters of the proposed model with a brief introduction of the LW model. Section 4 verifies the performance of the proposed model and discusses implied consumption dynamics and preferences. Section 5 introduces jumps into cash flow processes to obtain more plausible economic implications. Section 6 concludes. Technical arguments are collected in Appendices. 


\section{Model}

Our model is built on a simple exchange economy, where the flow of endowments is exogenously provided and a rational, representative agent has recursive utility of Epstein and Zin (1989, 1991), and Weil (1989). Section 2.1 first specifies the utility function and then extends it in a way where the parameters of risk aversion and time preference depend on state variables of the economy. Sections 2.2 through 2.4 specify endowment process and state-dependent preferences such that the recursive equation for the agent's continuation value is solved in closed form for a certain case, which is presented in Section 2.5. Section 2.6 derives an analytical approximation of the continuation value for a general case, guided by the results of Section 2.5. Finally, Section 2.7 provides pricing formulas for zero-coupon bonds and equities. The derivation of key equations are provided in Appendix A.

\section{$2.1 \quad$ Preference}

Let $U_{t}$ denote time- $t$ utility of the representative agent. It is originally specified by the following recursive form:

$$
U_{t}=\left\{(1-\beta) C_{t}^{\rho}+\beta E_{t}\left[U_{t+1}^{1-\gamma}\right]^{\rho /(1-\gamma)}\right\}^{1 / \rho},
$$

where $C_{t}$ is aggregate consumption at time $t$ to be determined by the agent (the decision variable), and $E_{t}[\cdot]$ stands for expectation conditioned on time $t$. There are three parameters in $U_{t}$ : $\beta$ represents time preference or subjective discount factor (typically somewhat less than one); $\gamma$ is a coefficient of risk aversion; and $\rho$ is related to the elasticity of intertemporal substitution (EIS) by $\mathrm{EIS}=1 /(1-\rho)$.

To capture average term structures of zero-coupon bonds and equities, we let $\beta$ and $\gamma$ be state-dependent as in Melino and Yang (2003). Unlike their study, we set $\rho$ to zero or equivalently the EIS to unity. This restriction has a merit of keeping the model simple without scarifying the goodness-of-fit to at least average term structures. The unit EIS is considered by Piazzesi and Schneider (2006) in modeling the term structure of interest rates. Hansen et al. (2007) show that a model with unit EIS can be used as a basis for approximating more general models.

By substituting $\left(\beta_{t}, \gamma_{t}\right)$ for $(\beta, \gamma)$ and $\rho=0$ in $(1)$,

$$
U_{t}=C_{t}^{1-\beta_{t}} E_{t}\left[U_{t+1}^{1-\gamma_{t}}\right]^{\beta_{t} /\left(1-\gamma_{t}\right)} .
$$

In solving the optimal consumption problem, we place the following assumptions: (i) $\beta_{t}$ and $\gamma_{t}$ are exogenous; and (ii) $0<\beta_{t}<1$ for all $t$. Assumption (i) is also considered by Gordon and 
St-Amour (2004), who model the risk-aversion coefficient directly by a stochastic process. The analogous assumption is made by Campbell and Cochrane (1999) in the form of external habit. Since $\beta_{t}$ and $\gamma_{t}$ are not affected by the decision variable, the optimal consumption problem can be solved in the same way as in the case of constant preference parameters. Assumption (ii) guarantees that the period utility in (2) is concave with respect to the decision variable and that the wealth is positive in equilibrium.

Let $C_{t}^{e}>0$ be time- $t$ endowment and $W_{t}$ be time- $t$ wealth, which in the endowment economy is the cum-dividend value of a claim to the flow of endowments. The gross rate of return to wealth, $R_{w, t+1}$, is defined by

$$
R_{w, t+1}=\frac{W_{t+1}}{W_{t}-C_{t}^{e}}
$$

Then, the budget constraint for the agent is

$$
R_{w, t+1}\left(W_{t}-C_{t}\right)=W_{t+1}
$$

Let $V_{t}$ be the continuation value, which is the solution to the following problem:

$$
V_{t}=\max _{C_{t}} U_{t} \quad \text { subject to }(4)
$$

Because of the unit EIS, the optimal consumption, $C_{t}^{*}$, has a closed form, irrespective of how $\beta_{t}$ and $\gamma_{t}$ are specified:

$$
C_{t}^{*}=\left(1-\beta_{t}\right) W_{t}
$$

Unlike a constant-parameter case, the wealth-consumption ratio, $W_{t} / C_{t}^{*}$, varies over time even for a unit EIS.

The equilibrium condition is that the agent consumes the given endowment, $C_{t}^{*}=C_{t}^{e}$. The wealth in equilibrium, $W_{t}^{*}$, is then solved as $W_{t}^{*}=\frac{1}{1-\beta_{t}} C_{t}^{e}$ from (6). By substituting $W_{t}^{*}$ into (3), the equilibrium, gross rate of return to wealth, $R_{w, t+1}^{*}$, is

$$
R_{w, t+1}^{*}=\frac{1}{\beta_{t}} \frac{1-\beta_{t}}{1-\beta_{t+1}} \frac{C_{t+1}^{e}}{C_{t}^{e}}
$$

The continuation value in equilibrium, $V_{t}^{*}$, is the solution to the following recursive equation: Define $\nu_{t}=V_{t}^{*} / C_{t}^{e}$, and $\nu_{t}$ satisfies $^{2}$

$$
\nu_{t}=E_{t}\left[\left(\nu_{t+1} \frac{C_{t+1}^{e}}{C_{t}^{e}}\right)^{1-\gamma_{t}}\right]^{\beta_{t} /\left(1-\gamma_{t}\right)} .
$$

\footnotetext{
${ }^{2}$ We call $\nu_{t}$ the continuation value unless otherwise noted because $V_{t}^{*}$ does not explicitly appear in discussions to follow.
} 
The SDF, $M_{t+1}$, is obtained as

$$
M_{t+1}=\beta_{t} \frac{1-\beta_{t+1}}{1-\beta_{t}}\left(\frac{\nu_{t+1}}{\nu_{t}^{1 / \beta_{t}}}\right)^{1-\gamma_{t}}\left(\frac{C_{t+1}^{e}}{C_{t}^{e}}\right)^{-\gamma_{t}} .
$$

In general, the recursive equation for $\nu_{t}$ cannot be solved in closed form, which makes the SDF unavailable in closed form. In the next subsections, we specify endowment process and statedependent preferences in a way where $\nu_{t}$ is solved in closed form for constant time preference and approximately for state-dependent time preference, keeping the accuracy in mind.

\subsection{Dynamics}

Following LW (2011), all variables are assumed to be homoscedastic. Define $c_{t}=\ln C_{t}^{e}$ and $\Delta c_{t+1}=c_{t+1}-c_{t}$. The evolution of rate of growth in endowment, or equivalently aggregate consumption in equilibrium, is specified as

$$
\Delta c_{t+1}=\mu_{c}+b_{c}^{\prime} x_{t}+\sigma_{c}^{\prime} z_{t+1}
$$

where $\mu_{c}$ is the unconditional mean of consumption growth rate (given that the unconditional mean of $x_{t}$ is zero), $x_{t}$ is a $d$-dimensional vector of state variables, and $z_{t+1}$ is a $(d+3)$-dimensional vector of i.i.d. normal random variables. The reason for the $(d+3)$ dimension will be clear soon.

For pricing nominal zero-coupon bonds, a general price index, $\Pi_{t}$, is introduced, which is assumed to be determined exogenously. Define $\pi_{t}=\ln \Pi_{t}$ and $\Delta \pi_{t+1}=\pi_{t+1}-\pi_{t}$. Then, the evolution of rate of growth in inflation is specified as

$$
\Delta \pi_{t+1}=\mu_{\pi}+b_{\pi}^{\prime} x_{t}+\sigma_{\pi}^{\prime} z_{t+1}
$$

where the parameters and variables are interpreted similarly with the consumption process in (10).

For pricing dividend strips, the flow of aggregate dividends needs to be specified. Let $D_{t}$ be aggregate divided payed at time $t$. Define $d_{t}=\ln D_{t}$ and $\Delta d_{t+1}=d_{t+1}-d_{t}$. Then, the evolution of rate of growth in dividend is specified as

$$
\Delta d_{t+1}=\mu_{d}+b_{d}^{\prime} x_{t}+\sigma_{d}^{\prime} z_{t+1}
$$

Aggregate dividend can be regarded as levered consumption in an endowment economy. The most direct description of this relation is $D_{t}=\left(C_{t}^{e}\right)^{a}$ for some constant $a>1$ (Abel, 1999; 
Campbell, 2003). Also, it is often assumed that $\Delta c_{t+1}$ and $\Delta d_{t+1}$ are co-integrated (Bansal, Gallant, and Tauchen, 2007). We consider their link in calibrating the parameters in Section 3.2

Finally, a $d$-dimensional state vector $x_{t}$ is assumed to follow

$$
x_{t+1}=\Phi_{x}^{\prime} x_{t}+\sigma_{x}^{\prime} z_{t+1}
$$

Note that the unconditional mean of $x_{t}$ is zero. There are totally $(d+3)$ variables in the economy.

For notational convenience, define $s_{i j}=\sigma_{i}^{\prime} \sigma_{j}$. For example, the covariance between innovations in $\Delta c_{t+1}$ and $\Delta d_{t+1}$ is denoted as $s_{c d}=\sigma_{c}^{\prime} \sigma_{d}$ (scaler). Likewise, the covariance between innovations in $\Delta c_{t+1}$ and $x_{t+1}$ is denoted as $s_{c x}=\sigma_{x}^{\prime} \sigma_{c}(d \times 1$ vector $)$ and the variance of innovation in $x_{t+1}$ as $s_{x x}=\sigma_{x}^{\prime} \sigma_{x}(d \times d$ matrix $)$.

\section{$2.3 \quad$ Risk aversion}

We specify the coefficient of risk aversion as a linear function of the state vector:

$$
\gamma_{t}=\mu_{\gamma}+b_{\gamma}^{\prime} x_{t}
$$

The linear specification has a merit of obtaining the SDF in closed form when time preference is constant. It is also useful for state-dependent time preference, which will be addressed in Section 2.6.

A caveat, on the other hand, is that since $x_{t}$ is Gaussian, $\gamma_{t}$ becomes negative with positive probability. This shortcoming is also seen in the previous work. Gordon and St-Amour (2004), and Dew-Becker (2014) specify $\gamma_{t}$ as a part of the VAR(1) system and an AR(1) process, respectively; however, they do not theoretically impose the positivity of $\gamma_{t}$. The probability of $\gamma_{t}<0$ in this study is addressed after calibrating the parameters in Section 4.1.

\subsection{Time preference}

We consider the following two specifications:

$$
\begin{aligned}
& \text { (S1) } \beta_{t}=\beta, \\
& \text { (S2) } \beta_{t}=1-\exp \left\{\mu_{\beta}+b_{\beta}^{\prime} x_{t}\right\} \quad\left(\mu_{\beta}<0\right) .
\end{aligned}
$$

S1 allows for solving the recursive equation (8) for $\nu_{t}$ in closed form. The resulting formula is exponentially linear in $x_{t}$, leading to the SDF of the affine class. The prices of zero-coupon 
bonds and equities are therefore available in closed form, which are also exponentially linear in $x_{t}$.

For any specification of $\beta_{t}$ except $\mathrm{S} 1, \nu_{t}$ has no closed form. To retain tractability, we perform an analytical approximation of $\nu_{t}$ in a way where the affine pricing framework is available as for S1. S2 is aimed at keeping the accuracy of the approximation, rather than based on economic reasoning or statistical adequacy. Specifically, once $\ln \nu_{t}$ is approximated as a linear function of $x_{t}$ (this approximation is inevitable for any specification of $\beta_{t}$ ), the price of risks is derived as a linear function of $x_{t}$ without further approximation. Intuitively, this is understood by noticing that the SDF given in (9) has a term $1-\beta_{t+1}$, which in S2 is exponentially linear in $x_{t+1}$. Also of note is that the wealth-consumption ratio given in (6) is log-linear in $x_{t}$.

A caveat of $\mathrm{S} 2$ is that $\beta_{t}$ becomes negative with positive probability, violating the lower bound constraint in Assumption (ii). The severity of this violation depends on parameter values and therefore is addressed after calibration in Section 4.1.

\subsection{SDF for S1}

We derive the continuation value and SDF for S1. Though our interest is in S2, the results for S1 are worth presenting for three reasons. First, they are an extension of the results presented by Hansen, Heaton and Li (2008). The extension is in the coefficient of risk aversion: It is constant in Hansen et al. (2008) whereas it is a linear function of Gaussian state variables in this study. Second, the fact that the SDF for S1 derived here is exact while that for S2 is approximate clarifies the source of approximation and provides the sense of accuracy. Third, through the comparison with $\mathrm{S} 1$, the extension to $\mathrm{S} 2$ can be highlighted.

The recursive equation (8) for $\nu_{t}$ is solved as

$$
\nu_{t}=\exp \left\{\mu_{\nu}+b_{\nu}^{\prime} x_{t}\right\}
$$

where $\mu_{\nu}$ and $b_{\nu}$ are the solutions to the following simultaneous equations:

$$
\begin{aligned}
\mu_{\nu} & =\beta\left\{\mu_{\nu}+\mu_{c}-\frac{1}{2} v_{c \nu}\left(\mu_{\gamma}-1\right)\right\}, \\
b_{\nu} & =\beta\left(b_{c}+\Phi_{x} b_{\nu}-\frac{1}{2} v_{c \nu} b_{\gamma}\right),
\end{aligned}
$$

where

$$
v_{c \nu}=\operatorname{var}_{t}\left[\ln \nu_{t+1}+\Delta c_{t+1}\right]=b_{\nu}^{\prime} s_{x x} b_{\nu}+2 s_{c x}^{\prime} b_{\nu}+s_{c c} .
$$


Note that (18) and (19) are quadratic equations because $v_{c \nu}$ is quadratic in $b_{\nu}$. Appendix B provides the condition for $\mu_{\nu}$ and $b_{\nu}$ to be real and addresses which real root to select. It is noted that setting $b_{\gamma}=0$ in (19) (i.e., a constant risk-aversion coefficient) leads to the continuation value presented by Hansen et al. (2008).

Next, we drive the price-of-risk vector, denoted as $\lambda_{t}$. It is the (negative) loading on the innovation vector $z_{t+1}$ in the SDF. By taking the log of (9) with $\beta_{t}$ replaced by $\beta$ and defining $m_{t+1}=\ln M_{t+1}$,

$$
m_{t+1}=\left(1-\gamma_{t}\right) \ln \nu_{t+1}-\gamma_{t} \Delta c_{t+1}+r e s_{t}^{m},
$$

where $\mathrm{res}_{t}^{m}$ collects the remaining terms observed at time $t$. By substituting (17) and then (10) and (13) into (21), we have $m_{t+1}-E_{t}\left[m_{t+1}\right]=-\lambda_{t}^{\prime} z_{t+1}$, where

$$
\lambda_{t}=\left(\sigma_{x} b_{\nu}+\sigma_{c}\right) \gamma_{t}-\sigma_{x} b_{\nu}
$$

Since $\gamma_{t}$ is assumed to be linear in $x_{t}$, so is $\lambda_{t}$. Owing to $\gamma_{t}$, the risk premium of any asset is also time-varying even without time-varying volatility. A potential drawback of $\lambda_{t}$ in (22) is that it is driven by $\gamma_{t}$ alone, which is a certain linear combination of the $d$-dimensional state vector as given in (14). This implies that the correlation between risk premiums of any pair of assets is one in absolute value as long as cash flow processes are homoscedastic. LW (2011) make a similar assumption that the price-of-risk vector is driven by one factor and point out the drawbacks of this assumption.

The one-period real risk-free rate, denoted as $r_{f, t+1}$, is the solution to the following Euler equation: $r_{f, t+1}=-\ln E_{t}\left[M_{t+1}\right]$. Then, it is also derived as a linear function of $x_{t}$ :

$$
r_{f, t+1}=A_{f}+B_{f}^{\prime} x_{t}
$$

where

$$
\begin{aligned}
& A_{f}=-\ln \beta+\mu_{c}-\frac{1}{2} s_{c c}-\left(s_{c x}^{\prime} b_{\nu}+s_{c c}\right)\left(\mu_{\gamma}-1\right), \\
& B_{f}=b_{c}-\left(s_{c x}^{\prime} b_{\nu}+s_{c c}\right) b_{\gamma} .
\end{aligned}
$$

Finally, $m_{t+1}$ can be rewritten in a conventional form as

$$
m_{t+1}=-r_{f, t+1}-\frac{1}{2} \lambda_{t}^{\prime} \lambda_{t}-\lambda_{t}^{\prime} z_{t+1}
$$

Since both $r_{f, t+1}$ and $\lambda_{t}$ are linear in $x_{t}, m_{t+1}$ falls into the affine class. 


\subsection{SDF for S2}

The recursive equation (8) for $\nu_{t}$ cannot be solved in closed form for a state-dependent time preference, which is denoted here as $\beta\left(x_{t}\right)$ to clarify the dependence on $x_{t}$. To retain tractability, we approximate $\nu_{t}$ by an exponentially linear function of $x_{t}$. To begin with, $\beta\left(x_{t}\right)$ and $\beta\left(x_{t}\right) x_{t}$ are linearized around $x_{t}=0$ (the unconditional mean):

$$
\begin{aligned}
\beta\left(x_{t}\right) & \approx \beta_{0}+\beta_{1}^{\prime} x_{t}, \\
\beta\left(x_{t}\right) x_{t} & \approx \beta_{0} x_{t},
\end{aligned}
$$

where $\beta_{0}=\beta(0)$ and $\beta_{1}=\left.\frac{d \beta\left(x_{t}\right)}{d x_{t}}\right|_{x_{t}=0}$. For S2, they are, respectively, $\beta_{0}=1-e^{\mu_{\beta}}$ and $\beta_{1}=-e^{\mu_{\beta}} b_{\beta}$. Then, $\nu_{t}$ is approximated by an exponentially linear function of $x_{t}$ as given in (17), where the coefficients satisfy the following simultaneous quadratic equations:

$$
\begin{aligned}
\mu_{\nu} & =\beta_{0}\left\{\mu_{\nu}+\mu_{c}-\frac{1}{2} v_{c \nu}\left(\mu_{\gamma}-1\right)\right\} \\
b_{\nu} & =\beta_{0}\left(b_{c}+\Phi_{x} b_{\nu}-\frac{1}{2} v_{c \nu} b_{\gamma}\right)+\beta_{1}\left\{\mu_{\nu}+\mu_{c}-\frac{1}{2} v_{c \nu}\left(\mu_{\gamma}-1\right)\right\},
\end{aligned}
$$

where $v_{c \nu}$ is given in (20). Notice that the second term on the RHS of (30) is newly added by the extension to S2.

The accuracy of the approximation to $\nu_{t}$ is examined in Appendix C. In brief, it seems to be maintained for parameter values determined by the calibration procedure in Section 3.2 and given specifically in Tables 2 and 4. Intuitively, the reason for a high accuracy is that $\beta\left(x_{t}\right)$ does not change much, as will be addressed in Section 4.1 and Figure 1(b). Then, (27) and (28) are not bad approximations after all.

Once $\ln \nu_{t}$ is approximated as a linear function of $x_{t}$, the price-of-risk vector $\lambda_{t}$ is derived as linear in $x_{t}$ without further approximation, which is due to $\mathrm{S} 2$ together with a linear specification of $\gamma_{t}$. Specifically, from (9), the log SDF can be written as

$$
m_{t+1}=\ln \left(1-\beta_{t+1}\right)+\left(1-\gamma_{t}\right) \ln \nu_{t+1}-\gamma_{t} \Delta c_{t+1}+r e s_{t}^{m},
$$

where $\mathrm{res}_{t}^{m}$ collects the remaining terms observed at time $t$. By substituting (16) and (17) and then (10) and (13) into (31), we have as before $m_{t+1}-E_{t}\left[m_{t+1}\right]=-\lambda_{t}^{\prime} z_{t+1}$, where

$$
\lambda_{t}=\left(\sigma_{x} b_{\nu}+\sigma_{c}\right) \gamma_{t}-\sigma_{x}\left(b_{\beta}+b_{\nu}\right)
$$

By a linear specification of $\gamma_{t}$ given in (14), $\lambda_{t}$ is also linear in $x_{t}$. Apart from $b_{\nu}$ that is not the same between $\mathrm{S} 1$ and $\mathrm{S} 2,-\sigma_{x} b_{\beta}$ is newly added by the extension to state-dependent time preference. 
Finally, to obtain the one-period real risk-free rate $r_{f, t+1}$ as a linear function of $x_{t}$, we need to rely on another approximation, which is to linearize $\ln \beta\left(x_{t}\right)$ around $x_{t}=0$. Specifically for $\mathrm{S} 2$,

$$
\ln \left(1-\exp \left\{\mu_{\beta}+b_{\beta}^{\prime} x_{t}\right\}\right) \approx \ln \left(1-e^{\mu_{\beta}}\right)-\frac{e^{\mu_{\beta}}}{1-e^{\mu_{\beta}}} b_{\beta}^{\prime} x_{t} .
$$

Again, this approximation may not be a serious concern due to a small variation in $\beta\left(x_{t}\right)$ as noted above. Then, $r_{f, t+1}$ is approximated as given in (23), where the coefficients are given as follows:

$$
\begin{aligned}
& A_{f}=-\ln \beta_{0}+\mu_{c}-\frac{1}{2} v_{c \beta}-\left(\mu_{\gamma}-1\right)\left(s_{c x}^{\prime} b_{\nu}+s_{c c}\right)+\left(\mu_{\gamma}-1\right)\left(s_{x x} b_{\nu}+s_{c x}\right)^{\prime} b_{\beta}, \\
& B_{f}=b_{c}-\left(s_{c x}^{\prime} b_{\nu}+s_{c c}\right) b_{\gamma}+\left\{\frac{1}{\beta_{0}} I_{d \times d}-\Phi_{x}+b_{\gamma}\left(s_{x x} b_{\nu}+s_{c x}\right)^{\prime}\right\} b_{\beta},
\end{aligned}
$$

where $I_{d \times d}$ is a $d$-by- $d$ identity matrix and

$$
v_{c \beta}=\operatorname{var}_{t}\left[\ln \left(1-\beta_{t+1}\right)-\Delta c_{t+1}\right]=b_{\beta}^{\prime} s_{x x} b_{\beta}-2 s_{c x}^{\prime} b_{\beta}+s_{c c}
$$

\subsection{Prices of zero-coupon bonds and equites}

Both the risk-free rate and price of risks are derived as linear functions of the Gaussian state vector exactly for S1 and approximately for S2. We now turn to the pricing of zero-coupon bonds and equites by utilizing the affine framework.

\subsubsection{Real zero-coupon bonds}

Let $P_{t, n}^{R}$ be time- $t$ real price of a zero-coupon bond maturing in time $t+n$ with the face value normalized to one unit of consumption. The Euler equation for $P_{t, n}^{R}$ is $P_{t, n}^{R}=E_{t}\left[M_{t+1} P_{t+1, n-1}^{R}\right]$ with the initial condition $P_{t, 0}^{R}=1$. The solution is of the form

$$
P_{t, n}^{R}=\exp \left\{A_{n}^{R}+B_{n}^{R \prime} x_{t}\right\}
$$

where $A_{n}^{R}$ and $B_{n}^{R}$ are determined recursively, starting with $A_{0}^{R}=0$ and $B_{0}^{R}=0$. The recursive equations are provided in Appendix D.

\subsubsection{Nominal zero-coupon bonds}

Let $P_{t, n}^{N}$ be time- $t$ real price of a zero-coupon bond maturing in time $t+n$ with the face value normalized to one in nominal terms or equivalently $1 / \Pi_{t+n}$ in real terms. The Euler equation 
for $P_{t, n}^{N}$ is $P_{t, n}^{N}=E_{t}\left[M_{t+1} P_{t+1, n-1}^{N}\right]$ with the initial condition $P_{t, 0}^{N} \Pi_{t}=1$. It follows that

$$
P_{t, n}^{N} \Pi_{t}=E_{t}\left[M_{t+1}\left(P_{t+1, n-1}^{N} \Pi_{t+1}\right) \frac{\Pi_{t}}{\Pi_{t+1}}\right] .
$$

The solution to (38) is of the form

$$
P_{t, n}^{N} \Pi_{t}=\exp \left\{A_{n}^{N}+B_{n}^{N \prime} x_{t}\right\}
$$

where $A_{n}^{N}$ and $B_{n}^{N}$ are determined recursively, starting with $A_{0}^{N}=0$ and $B_{0}^{N}=0$. The recursive equations are provided in Appendix D.

\subsubsection{Zero-coupon equities or divided strips}

Let $P_{t, n}^{D}$ be time- $t$ real price of a zero-coupon equity that pays $D_{t+n}$ in time $t+n$. The Euler equation for $P_{t, n}^{D}$ is $P_{t, n}^{D}=E_{t}\left[M_{t+1} P_{t+1, n-1}^{D}\right]$ with the initial condition $P_{t, 0}^{D} / D_{t}=1$. It follows that

$$
\frac{P_{t, n}^{D}}{D_{t}}=E_{t}\left[M_{t+1} \frac{P_{t+1, n-1}^{D}}{D_{t+1}} \frac{D_{t+1}}{D_{t}}\right]
$$

The solution to (40) is of the form

$$
P_{t, n}^{D} / D_{t}=\exp \left\{A_{n}^{D}+B_{n}^{D \prime} x_{t}\right\}
$$

where $A_{n}^{D}$ and $B_{n}^{D}$ are determined recursively, starting with $A_{0}^{D}=0$ and $B_{0}^{D}=0$. The recursive equations are provided in Appendix D.

\section{Calibration}

We calibrate the parameters of the proposed model by matching it with the LW model. Specifically, both the one-period real risk-free rate $r_{f, t+1}$ and the price-of-risk vector $\lambda_{t}$ are matched between the two models, which means from equation (26) that the SDF of the LW model that is exogenously specified is replicated by that of the proposed model endogenously derived from equilibrium conditions. Both models then agree with the price of any asset. This calibration approach has two advantages. First, it provides the proposed model with the opportunity to inherit a high descriptive ability of the LW model with respect to average term structures of zero-coupon bonds and equities. Second, it provides the LW model with an equilibrium foundation, uncovering preferences and consumption dynamics implicit in this reduced-form model. Section 3.1 introduces the LW model and Section 3.2 explains a calibration procedure. 


\subsection{The LW model}

The LW model has the following six variables (the notation is slightly changed from the original one):

$$
\begin{aligned}
\Delta d_{t} & : \text { divided growth rate } \\
\Delta \pi_{t} & : \text { inflation growth rate } \\
x_{d, t} & : \text { factor driving expected divided growth rate } \\
x_{\pi, t} & : \text { factor driving expected inflation growth rate } \\
x_{f, t} & : \text { factor driving the real risk-free rate } \\
x_{\lambda, t} & : \text { factor driving the price of risks }
\end{aligned}
$$

Note that consumption growth rate does not appear in the LW model as it is a reduced-form model. The last four variables are collected in a state vector, denoted as $x_{t}^{L W}$ :

$$
x_{t}^{L W}=\left(x_{d, t} \quad x_{\pi, t} \quad x_{f, t}-\mu_{f} \quad x_{\lambda, t}-\mu_{\lambda}\right)^{\prime},
$$

where $\mu_{f}$ and $\mu_{\lambda}$ are the unconditional means of $x_{f, t}$ and $x_{\lambda, t}$, respectively (those of $x_{d, t}$ and $x_{\pi, t}$ are implicitly assumed to be zero). The dynamics of these variables are specified as

$$
\begin{aligned}
\Delta d_{t+1} & =\mu_{d}+x_{d, t}+\sigma_{d}^{\prime} z_{t+1}, \\
\Delta \pi_{t+1} & =\mu_{\pi}+x_{\pi, t}+\sigma_{\pi}^{\prime} z_{t+1}, \\
x_{t+1}^{L W} & =\Phi_{x}^{L W \prime} x_{t}^{L W}+\sigma_{x}^{L W \prime} z_{t+1} .
\end{aligned}
$$

The log SDF of the LW model, denoted as $m_{t+1}^{L W}$, is specified exogenously as

$$
m_{t+1}^{L W}=-x_{f, t}-\frac{1}{2} s_{d d} x_{\lambda, t}^{2}-x_{\lambda, t} \sigma_{d}^{\prime} z_{t+1},
$$

where $s_{d d}=\sigma_{d}^{\prime} \sigma_{d}$ A notable feature of $m_{t}^{L W}$ is that it is driven by the same innovation term as driving dividend growth, $\sigma_{d}^{\prime} z_{t+1}$. The conditional correlation between $m_{t+1}^{L W}$ and $\Delta d_{t+1}$ is then $-x_{\lambda, t} /\left|x_{\lambda, t}\right|$. Since the parameters calibrated by LW imply $\operatorname{Pr}\left\{x_{\lambda, t}>0\right\}=0.99$, these two variables can safely be regarded as perfectly negatively correlated. That is, a negative shock to dividend growth almost always increases the SDF. This mechanism is the key to generating high risk premiums of short-term dividend strips that are strongly affected by shocks to dividend growth. Risk premiums arising from shocks to the other variables are non-zero as long as these shocks have non-zero correlations with dividend growth shock.

The parameter values of the LW model are summarized in Table 2 of this manuscript, which are collected from tables 1-3 in LW (2011). The unconditional means, standard deviations, and 
autocorrelations are expressed in annual terms, except for conditional first and second moments of $x_{\lambda, t}$ expressed in raw numbers. The annual numbers are transformed into quarterly raw numbers when substituted into the models.

Several notes on the parameter values are in order. First, the autoregressive matrix of $x_{t}^{L W}, \Phi_{x}^{L W}$, is diagonal. The expected-dividend-growth factor $x_{d, t}$ and the risk-free rate factor $x_{f, t}$ are relatively persistent as the autoregressive coefficients are equal to and larger than 0.9 , respectively. Second, the correlation between innovations in $\Delta d_{t}$ and $x_{d, t}$ is -0.83 , indicating that a negative shock to realized dividend growth is more likely increases expected dividend growth. An important implication of the negative correlation is that long-term dividend strips are not as risky as short-term ones because a negative shock to realized dividend growth, which always increases the SDF, raises the level of future dividends and thus the price of long-term dividend strips. The negative correlation together with the innovation term of the SDF given in (46) are the key for a downward-sloping term structure of dividend risk premiums. Third, the correlation between innovations in $\Delta \pi_{t}$ and $x_{\pi, t}$ is set to one, indicating that realized and expected inflation growth rates move one for one. Fourth, the correlation between innovations in $\Delta d_{t}$ and $\pi_{t}$ is -0.3 . Because $\pi_{t}$ and $x_{\pi, t}$ are perfectly correlated, the correlation between innovations in $\Delta d_{t}$ and $x_{\pi, t}$ is also -0.3 . Then, a positive shock to realized and expected inflation growth more likely decreases realized dividend growth, which increases the SDF. Meanwhile, the rise in realized and expected inflation growth lowers the payoffs of nominal bonds in real terms with both short and long maturities. Hence, nominal bonds cannot be hedge against events of raising the SDF, leading to an upward-sloping term structure of nominal interest rates. Fifth, the correlation between innovations in $\Delta d_{t}$ and $x_{f, t}$ is -0.3 . The (weak) negative correlation contributes to generating a moderately upward-sloping term structure of real interest rates. Specifically, by a negative shock to realized dividend growth, the SDF increases and the real risk-free rate tends to rise by the negative correlation. The rise in the real risk-free rate in turn lowers the prices of real bonds, indicating that real bonds cannot be hedge against events of raising the SDF.

In summary, all variables, except $x_{\lambda, t}$, are correlated negatively with $\Delta d_{t}$ and hence positively with $m_{t}$, which intuitively means that the agent dislikes increase in these variables. The factor risk premiums, which are computed as $-\operatorname{cov}_{t}\left[m_{t+1}, \cdot\right]=s_{d} \cdot x_{\lambda, t}$, are then negative except those of $\Delta d_{t}$ and $x_{\lambda, t}$. In the last row of Table 2 , the factor risk premiums evaluated at $x_{\lambda, t}=\mu_{\lambda}$ are presented in annual percentage terms. First, by far the largest in absolute value is the factor 
risk premium of $\Delta d_{t}, 17 \%$ per year. Then, an asset that has a positive exposure to $\Delta d_{t}$, such as short-term dividend strips, is supposed to command a positive risk premium. In fact, the risk premium of the one-quarter dividend strip is exactly $17 \%$. Second, the factor risk premium of $x_{\lambda, t}$ is zero by the zero correlation between innovations in $x_{\lambda, t}$ and $\Delta d_{t}$. Then, even though an asset has either a positive or negative exposure to $x_{\lambda, t}$, this does not affect the risk premium of this asset. However, the exposure to $x_{\lambda, t}$ does affect the volatility and thus the Sharpe ratio of this asset. Third, the factor risk premiums of the rest of the variables are negative. Then, an asset that has a positive exposure to one of these variables commands a negative risk premium attributed to it.

\subsection{Calibration procedure}

The most straightforward approach for replicating the SDF of the LW model with that of the proposed model is to use the same variables. Specifically, we match $x_{t}=x_{t}^{L W}$ and inherit the dynamics of $x_{t}^{L W}$ as well as those of $\Delta d_{t}$ and $\Delta \pi_{t}$ into the proposed model. This means that the parameters associated with these dynamics are not calibrated in this study: We simply borrow them from LW (2011). Then, the parameters to calibrate here are those associated with consumption dynamics and state-dependent preferences that do not appear in the LW model.

We first re-specify the consumption process as

$$
\Delta c_{t+1}=\mu_{c}+b_{c} x_{d, t}+\sigma_{c}^{\prime} z_{t+1}
$$

which is similar to the dividend process given in (43). In particular, expected consumption growth is driven by the same state variable (scaled by $b_{c}$ ) as driving expected dividend growth. The parameters of the consumption process are those of the drift, $\left(\mu_{c}, b_{c}\right)$, and those associated with the variance and covariances with the other six variables, $\left(s_{c c}, s_{c d}, s_{c \pi}, s_{c x}^{\prime}\right)$. Among these consumption parameters, we fix $\left(\mu_{c}, b_{c}\right)$ to maintain a reasonable relationship between consumption and dividend growth (a more detailed reason is provided below). Specifically, we set $\mu_{c}=\mu_{d}$ and $b_{c}=1 / 3$, following Bansal and Yaron (2004). Then, there are seven consumption parameters that need to be calibrated.

On the other hand, the parameters of state-dependent preferences are $\left(\mu_{\gamma}, b_{\gamma}^{\prime}\right)$ in the riskaversion coefficient $\gamma_{t}$ and $\left(\mu_{\beta}, b_{\beta}^{\prime}\right)$ in the subjective discount factor $\beta_{t}$. Among these preference parameters, some elements of $b_{\gamma}$ can be determined immediately. In both the LW and proposed models, the price-of-risk vector is driven by one factor: $x_{\lambda, t}$ in the LW model and $\gamma_{t}$ in the 
proposed model. It is deduced from this relation that $\gamma_{t}$ is a linear function of $x_{\lambda, t}$ alone:

$$
\gamma_{t}=\mu_{\gamma}+b_{\gamma 4}\left(x_{\lambda, t}-\mu_{\lambda}\right)
$$

Hence, $b_{\gamma i}=0(i=1,2,3)$, resulting in seven preference parameters that need to be calibrated.

These unknown parameters are determined by numerically solving the following sets of constraint equations. The first set is obtained by matching the one-period real risk-free rate, which is given as a state variable in the LW model and as a linear function of state variables in the proposed model. Specifically,

$$
x_{f, t}=A_{f}+B_{f 1} x_{d, t}+B_{f 2} x_{\pi, t}+B_{f 3}\left(x_{f, t}-\mu_{f}\right)+B_{f 4}\left(x_{\lambda, t}-\mu_{\lambda}\right) .
$$

Equation (49) holds for any $x_{t}$, leading to the five constraint equations:

$$
B_{f 1}=B_{f 2}=B_{f 4}=0, \quad B_{f 3}=1, \quad A_{f}=\mu_{f} .
$$

The second set of constrained equations is obtained by matching the factor risk premiums. In the LW model (augmented with the consumption process given in (47)):

$$
\begin{aligned}
-\operatorname{cov}_{t}\left[m_{t+1}^{L W}, \Delta c_{t+1}\right] & =s_{c d} x_{\lambda, t}, \\
-\operatorname{cov}_{t}\left[m_{t+1}^{L W}, \Delta d_{t+1}\right] & =s_{d d} x_{\lambda, t}, \\
-\operatorname{cov}_{t}\left[m_{t+1}^{L W}, \Delta \pi_{t+1}\right] & =s_{d \pi} x_{\lambda, t}, \\
-\operatorname{cov}_{t}\left[m_{t+1}^{L W}, x_{t+1}\right] & =s_{d x} x_{\lambda, t} .
\end{aligned}
$$

Note that (54) is four dimensional. The corresponding factor risk premiums in the proposed model are

$$
\begin{aligned}
-\operatorname{cov}_{t}\left[m_{t+1}, \Delta c_{t+1}\right] & =\left(s_{c x}^{\prime} b_{\nu}+s_{c c}\right) \gamma_{t}-s_{c x}^{\prime}\left(b_{\beta}+b_{\nu}\right), \\
-\operatorname{cov}_{t}\left[m_{t+1}, \Delta d_{t+1}\right] & =\left(s_{d x}^{\prime} b_{\nu}+s_{c d}\right) \gamma_{t}-s_{d x}^{\prime}\left(b_{\beta}+b_{\nu}\right), \\
-\operatorname{cov}_{t}\left[m_{t+1}, \Delta \pi_{t+1}\right] & =\left(s_{\pi x}^{\prime} b_{\nu}+s_{c \pi}\right) \gamma_{t}-s_{\pi x}^{\prime}\left(b_{\beta}+b_{\nu}\right), \\
-\operatorname{cov}_{t}\left[m_{t+1}, x_{t+1}\right] & =\left(s_{x x}^{\prime} b_{\nu}+s_{c x}\right) \gamma_{t}-s_{x x}^{\prime}\left(b_{\beta}+b_{\nu}\right) .
\end{aligned}
$$

By substituting (48) into (55)-(58) and then matching the resulting equations with (51)-(54), we have the following fourteen equations:

$$
\begin{array}{ll}
\text { (Slope) } & \text { (Intersept) } \\
b_{\gamma 4}\left(s_{c x}^{\prime} b_{\nu}+s_{c c}\right)=s_{c d}, & \left(\mu_{\gamma}-b_{\gamma 4} \mu_{\lambda}\right)\left(s_{c x}^{\prime} b_{\nu}+s_{c c}\right)-s_{c x}^{\prime}\left(b_{\beta}+b_{\nu}\right)=0,
\end{array}
$$




$$
\begin{array}{ll}
b_{\gamma 4}\left(s_{d x}^{\prime} b_{\nu}+s_{c d}\right)=s_{d d}, & \left(\mu_{\gamma}-b_{\gamma 4} \mu_{\lambda}\right)\left(s_{d x}^{\prime} b_{\nu}+s_{c d}\right)-s_{d x}^{\prime}\left(b_{\beta}+b_{\nu}\right)=0 \\
b_{\gamma 4}\left(s_{\pi x}^{\prime} b_{\nu}+s_{c \pi}\right)=s_{d \pi}, & \left(\mu_{\gamma}-b_{\gamma 4} \mu_{\lambda}\right)\left(s_{\pi x}^{\prime} b_{\nu}+s_{c \pi}\right)-s_{\pi x}^{\prime}\left(b_{\beta}+b_{\nu}\right)=0 \\
b_{\gamma 4}\left(s_{x x}^{\prime} b_{\nu}+s_{c x}\right)=s_{d x}, & \left(\mu_{\gamma}-b_{\gamma 4} \mu_{\lambda}\right)\left(s_{x x}^{\prime} b_{\nu}+s_{c x}\right)-s_{x x}^{\prime}\left(b_{\beta}+b_{\nu}\right)=0 .
\end{array}
$$

Taken together, there are fourteen unknown parameters; two in $\gamma_{t}$, five in $\beta_{t}$, and seven for consumption variance and covariances. Meanwhile, there are nineteen constraint equations needed for perfect replication; five from the risk-free rate and fourteen from the factor risk premiums. Hence, the perfect replication is impossible in the first place. This is so even if $\left(\mu_{c}, b_{c}\right)$ are free parameters. In this case, these drift parameters are used for matching the factor risk premiums, rather than capturing expected consumption growth. Consequently, unrealistic values are returned, and this is why we fix $\left(\mu_{c}, b_{c}\right)$ for a realistic consumption process.

Among these equations, five equations in (50) and seven slope equations in (59)-(62) are selected. By this selection, there is no difference in the loadings of each asset on the state vector, $B_{n}^{i}(i=\{R, N, D\})$, between the LW and proposed models; see Appendix D for detail. Additionally, given the fact that the factor risk premium of $\Delta d_{t}$ is by far the largest, the intercept equation (60) is also selected. This means that the factor risk premium for $\Delta d_{t}$ is exactly matched between the two models, and so is the risk premium of the one-quarter dividend strip (17\% per year). Finally, one free parameter is reserved for keeping positive definite the extended correlation matrix, which includes consumption growth but excludes realized inflation growth due to the perfect correlation with expected inflation growth. Without this constraint, a negative definite correlation matrix is returned in exchange for a closer fit to the SDF of the LW model.

It is noted that since the rest of the intercept equations, (59) and (61)-(62), are not satisfied, average term structures differ between the two models, as will be seen in Section 4.3. There are two reasons that we give priority to the slope equations over the intercept equations. First, the constant terms in the pricing formulas, $A_{n}^{i}(i=\{R, N, D\})$, which matter with average term structures, are computed recursively and dependently on the loadings; see equations (144), (147), and (150) in Appendix D. Second, it is difficult to find the solutions to intercept equations that satisfy the following two conditions: (i) $\left(\mu_{\nu}, b_{\nu}\right)$ are real; and (ii) the extended correlation matrix is positive definite. 


\section{Baseline results}

The purpose of this section is to address whether the proposed model can replicate the ter$\mathrm{m}$ structures of bonds and equities generated by the LW model and discuss preferences and consumption dynamics implicit in these term structures. There are multiple, in fact numerous, solutions to the set of constraint equations presented in Section 3.2. In Section 4.1, we first select several solutions to discuss their pattern, and then detail one of them in Section 4.2. At this particular solution, we generate term structures of zero-coupon bonds and equities in Section 4.3. Finally, in section 4.4, we change the shape of the average term structure of real interest rates without much affecting the other term structures. This ability is important as the shape of the real term structure is indecisive.

\subsection{Several solutions and their pattern}

Table 3 presents several solutions in ascending order of the mean risk aversion $\mu_{\gamma}$. First of all, there is an inverse relationship between $\mu_{\gamma}$ and the volatility of innovation in consumption growth $\sqrt{s_{c c}}$. In addition, this relationship is nonlinear: The rate of decrease in $\sqrt{s_{c c}}$ is much slower than the rate of increase in $\mu_{\gamma}$. Specifically, at $\mu_{\gamma}=30, \sqrt{s_{c c}}$ is $8.84 \%$ per year, which is large relative to historical estimates discussed below and the corresponding dividend volatility set at $10 \%$. It becomes half at around $\mu_{\gamma}=120$ and less than $4 \%$ at $\mu_{\gamma}=150$. Further reduction in $\sqrt{s_{c c}}$ is limited: $\left(\sqrt{s_{c c}}, \mu_{\gamma}\right)=(3.43 \%, 300),(3.32 \%, 500),(3.28 \%, 1000)$.

Second, the unconditional standard deviation of the risk aversion, $S D\left[\gamma_{t}\right]$, also increases with $\mu_{\gamma}$. In fact, the ratio of mean to standard deviation is nearly constant at 1.2 for any solutions. Because the standard deviation is large relative to the mean, $\gamma_{t}$ becomes negative with a nonnegligible probability. Figure 1(a) depicts the unconditional distribution of $\gamma_{t}$ at Solution (e) of Table 3 (i.e., $\mu_{\gamma}=150$ ). The probability of $\gamma_{t}<0$ reaches $12 \%$. The result may be in line with Curatola (2015) in that risk loving agents exist at a certain proportion of the population, which helps explain a negative slope of dividend risk premiums.

If a negative risk aversion is unacceptable, it can be avoided by specifying $\gamma_{t}$ as a positive function of $x_{t}$. In Appendix C, we consider a quadratic specification and examine the accuracy of the approximation for the continuation value $\nu_{t}$. Alternatively, it is also possible to virtually avoid negative values by reducing the volatility of $x_{\lambda, t}$. It is originally set at 4 (raw figures) by LW (2011). But the results of this study suggest that it is too large. In Section 5, we change 
the values of some of the parameters originally set by LW to see whether reasonable economic implications are obtained without reducing the descriptive ability for average term structures presented in this section.

Third, the unconditional mean of the subjective discount factor, $E\left[\beta_{t}\right]$, expressed in quarterly terms, increases with $\mu_{\gamma}$. At $\mu_{\gamma}=30$, it is 0.969 , which appears to be smaller than usually considered. At $\mu_{\gamma}=90$, it increases to a reasonable value of 0.985 . Conversely, the unconditional standard deviation of the subjective discount factor, $S D\left[\beta_{t}\right]$, decreases with $\mu_{\gamma}$. The inverse relationship between the mean and variance of $\beta_{t}$ is a natural consequence of the specification given in (16), which has an upper bound of one. Figure 1(b) depicts the unconditional distribution of $\beta_{t}$ at Solution (e) of Table 3 (i.e., $\mu_{\gamma}=150$ ). Obviously, $\beta_{t}$ does not vary largely. Consequently, the unconditional probability of $\beta_{t}<0$ is negligibly small, indicating that the specification given in (16) is virtually consistent with Assumption (ii) (i.e., $0<\beta_{t}<1$ ). Also of note is that a small variation in $\beta_{t}$ is beneficial for the accuracy of the approximation to $\nu_{t}$. As shown in Section 2.6, the source of the approximation lies in (27) and (28). The smaller

the variation in $\beta_{t}$, the more accurate is the approximation. In the limit, if $\beta_{t}$ is constant, no approximation is involved as seen in Section 2.5.

In summary, through the matching of the proposed model with the LW model, it is revealed that we face either large risk aversion or high consumption volatility, or both. This is a typical tradeoff in the literature of equity premium puzzles. But here, it results from many equity risk premiums having the term structure with a sharply downward slope. The tradeoff implies that the specification of the SDF and/or the calibration of the parameters provided by LW (2011) are not in fact realistic from an equilibrium point of view. This problem is masked by the flexibility of the LW model. But once economic structures are imposed, it does emerge. In Section 5, we slightly change the specification of cash flow processes as well as the values of the parameters to resolve the trade-off and recover realistic economic implications.

\subsection{Implied consumption dynamics and preferences at a particular solution}

Facing the tradeoff between risk aversion and consumption volatility, we choose a reasonable consumption volatility while giving up a reasonable risk aversion. Specifically, we focus here on Solution (e) of Table 3, characterized by $\mu_{\gamma}=150$ and $\sqrt{s_{c c}}=3.91 \%$. The consumption volatility of $4 \%$ seems reasonable when viewed from U.S. historical data. For example, in Barro (2006, Table III), a sample standard deviation of real per capita GDP growth over 1890-2004 
is 4.5\%. In Mehra and Prescott (1985, table 1), originally from Grossman and Shiller (1981), a sample standard deviation of real per capita consumption growth over 1889-1978 is 3.6\%.

We report all calibrated parameters with particular attention to the following two points. The first is the mechanism of implying a large $\mu_{\gamma}$ and the second is key parameters for determining the shape of the average term structure of real interest rates. The parameters of consumption dynamics are first addressed, followed by those of state-dependent preferences.

\subsubsection{Consumption growth}

Panel A of Table 4 presents implied correlations between innovations in consumption growth $\Delta c_{t}$ and the other six variables in addition to the volatility of innovation in $\Delta c_{t}$. First, the correlation between $\Delta c_{t}$ and dividend growth $\Delta d_{t}$, denoted as $\rho_{c d}$, is 0.88. A high $\rho_{c d}$ is a natural consequence of the calibration procedure, which requires that the innovation term of the SDF in the LW model be replicated by innovations in consumption growth and associated state variables in the proposed model. The implied correlation is lower than it is in assuming $D_{t}=\left(C_{t}^{e}\right)^{a}$ : In this case $\rho_{c d}=1$. However, it may be larger than conventional estimates from time-series data on aggregate consumption and dividend growth. An economic rationale of this result seems to be limited participation in asset markets. Namely, as assumed by Marfè (2017), only shareholders who earn dividends and consume them can have access to the markets, indicating that the SDF is that of shareholders. This assumption then supports not only the SDF of the LW model but a high correlation between consumption and dividend growth.

Second, the correlation between $\Delta c_{t}$ and expected-dividend-growth factor $x_{d, t}$, denoted as $\rho_{c x 1}$, is -0.76 , which is close to the correlation between $\Delta d_{t}$ and $x_{d, t}$ originally fixed at -0.83 . These negative correlations contribute to making longer-term dividend strips less risky than shorter-term ones.

Third, the correlation between $\Delta c_{t}$ and expected-inflation-growth factor $x_{\pi, t}$, denoted as $\rho_{c x 2}$, is -0.09 , which is somewhat smaller in absolute value than the correlation between $\Delta d_{t}$ and $x_{\pi, t}$ originally fixed at -0.3 . Note that the correlation between $\Delta c_{t}$ and realized inflation growth $\Delta \pi_{t}$ is the same as $\rho_{c x 2}$ due to the perfect correlation between $\Delta \pi_{t}$ and $x_{\pi, t}$. Negative correlations of consumption growth with realizedl and expected inflation growth are the key channel through which equilibrium models generate a positive slope of the term structure of nominal interest rates (e.g., Piazzesi and Schneider, 2006; Wachter, 2006). Empirically, however, it may be difficult to find decisive evidence for a negative correlation. It is then beneficial to create alternative 
channels of making longer-term nominal bonds riskier. Indeed, the proposed model has such a channel, which is addressed in Section 4.4.

Fourth, the correlation between $\Delta c_{t}$ and risk-free-rate factor $x_{f, t}$, denoted as $\rho_{c x 3}$, is -0.05 . It is consistent in sign with, but smaller in magnitude than, the correlation between $\Delta d_{t}$ and $x_{f, t}$ originally fixed at -0.3 .

Finally, the correlation between $\Delta c_{t}$ and price-of-risk factor $x_{\lambda, t}$, denoted as $\rho_{c x 4}$, is 0.34 , which is higher than the correlation between $\Delta d_{t}$ and $x_{\lambda, t}$ originally fixed at zero. A positive $\rho_{c x 4}$ is from a positive covariance $s_{c x 4}$, which is needed to match the factor risk premium of $x_{\lambda, t}$ between the LW and proposed models. Specifically, recall that the fourth row of the slope equation $(62)$ is

$$
b_{\gamma 4}\left(s_{x x 4}^{\prime} b_{\nu}+s_{c x 4}\right)=s_{d x 4} \quad(=0),
$$

where $s_{c x 4}$ and $s_{d x 4}$ are, respectively, the fourth row of $s_{c x}$ and $s_{d x}$ (the vectors consisting of covariances of innovation in $x_{t}$ with innovations in $\Delta c_{t}$ and $\Delta d_{t}$ ) and $s_{x x 4}$ is the fourth column of $s_{x x}$ (the variance matrix of innovation in $x_{t}$ ). Note that $s_{d x 4}$ is originally set to zero by LW (2011). On the LHS of (63), $s_{x x 4}^{\prime} b_{\nu}=\operatorname{cov}_{t}\left[x_{\lambda, t+1}, \ln \nu_{t+1}\right]$ turns out to be negative, which is intuitive because a positive shock to the price of risks tends to lower the continuation value. It then follows that $s_{c x 4}=-s_{x x 4}^{\prime} b_{\nu}>0$.

A positive correlation between $\Delta c_{t}$ and $x_{\lambda, t}$ appears counterintuitive as a positive shock to consumption growth tends to raise the price of risks. Because a positive shock to consumption growth lowers the SDF, it may be concerned that the SDF lowers in response to rise in $x_{\lambda, t}$. We will show below that the SDF, in fact, is correlated positively with $x_{\lambda, t}$.

Taken together, implied parameters of the consumption process are basically consistent with pre-determined parameters of the divided process. Though the correlation between innovations in consumption growth and price of risks becomes positive, this is inevitable by the calibration procedure.

\subsubsection{Preferences}

To ease explanation, we call $1-\beta_{t}$ the subjective discount rate. Panel B of Table 4 presents implied parameters of the risk aversion $\gamma_{t}$ and the $\log$ subjective discount rate $\ln \left(1-\beta_{t}\right)$, together with calculated parameters of the $\log$ continuation value $\ln \nu_{t}$.

First of all, the loadings on the expected-inflation-growth factor $x_{\pi, t}$ are zero for all of these functions. The reason that $\gamma_{t}$ does not depend on $x_{\pi, t}$ is noted in Section 3.2. $x_{\pi, t}$ does not 
affect $\ln \left(1-\beta_{t}\right)$ because it does not enter into the risk-free rate by the constraint $B_{f 2}=0$ given in (50). Since $x_{\pi, t}$ has no influence on $\gamma_{t}, \beta_{t}$, or $\Delta c_{t}$, it has no channel of affecting $\nu_{t}$.

\section{Risk aversion}

As reported in Table 3, the standard deviation of $\gamma_{t}$ is 128 . Behind such a large value, $b_{\gamma 4}$ (the coefficient of $x_{\lambda, t}$ ) is implied to be 8.94. A large $b_{\gamma 4}$ together with a large $\mu_{\gamma}$ are a consequence of explaining a large factor risk premium of $\Delta d_{t}$ set by LW. Specifically, recall that the slope equation for matching the factor risk premium of $\Delta d_{t}$ is

$$
b_{\gamma 4}\left(s_{d x}^{\prime} b_{\nu}+s_{c d}\right)=s_{d d}
$$

On the LHS, $s_{d x}^{\prime} b_{\nu}=\operatorname{cov}_{t}\left[\Delta d_{t+1}, \ln \nu_{t+1}\right]$ turns out to be negative, indicating that a positive shock to dividend growth tends to decrease the continuation value. This relation can intuitively be understood by recalling that a positive shock to $\Delta d_{t}$ more likely reduces $x_{d, t}$ due to $\rho_{d x 1}=$ -0.83 and hence $\ln \nu_{t}$ having a positive coefficient of $x_{d, t}, b_{\nu 1}=8.86$. Meanwhile, both $b_{\gamma 4}$ and $s_{d d}$ in (64) are positive. It follows that $s_{c d}>-s_{d x}^{\prime} b_{\nu}>0$. But because $s_{c d}=\sqrt{s_{c c} s_{d d}} \rho_{c d}$ is much smaller than $s_{d d}$ given a reasonable $\sqrt{s_{c c}}$ (around $4 \%$ per year) and $\rho_{c d} \leq 1, b_{\gamma 4}$ must be large to equate the LHS with $s_{d d}$.

This logic can also be used for explaining why a large $\sqrt{s_{c c}}$ is required to reduce $b_{\gamma 4}$. For a small $b_{\gamma 4}, s_{c d}$ must be large to satisfy (64). But because $s_{d d}$ is fixed at $10 \%$ per year and because $\rho_{c d}$ has an upper limit of one, there is no other way but to increase $\sqrt{s_{c c}}$.

A large $b_{\gamma 4}$ leads to a large $\mu_{\gamma}$, which is explained using the intercept equation for matching the factor risk premium of $\Delta d_{t}$ :

$$
\left(\mu_{\gamma}-b_{\gamma 4} \mu_{\lambda}\right)\left(s_{d x}^{\prime} b_{\nu}+s_{c d}\right)=s_{d x}^{\prime}\left(b_{\beta}+b_{\nu}\right)
$$

The RHS of (65) is

$$
s_{d x}^{\prime}\left(b_{\beta}+b_{\nu}\right)=\operatorname{cov}_{t}\left[\Delta d_{t+1}, \ln \nu_{t+1}\right]+\operatorname{cov}_{t}\left[\Delta d_{t+1}, \ln \left(1-\beta_{t+1}\right)\right]
$$

As noted above, the first term on the RHS of (66) is negative. The second term on the RHS turns out to be positive but is dominated by the first term. Then, the RHS of (65) is negative but not very large in absolute value. Meanwhile, $b_{\gamma 4}$ on the LHS of (65) is large to satisfy (64) and $\mu_{\lambda}$ is originally fixed at 17 . To offset $b_{\gamma 4} \mu_{\lambda}, \mu_{\gamma}$ must also become large. 
These explanations make it clear why we face the tradeoff between reasonable values of risk aversion and consumption volatility. In order to reduce $\mu_{\gamma}, b_{\gamma 4}$ must also be reduced. But this is possible only by increasing the consumption volatility.

Because of $b_{\gamma 4}=8.94$, the risk aversion increases with the price-of-risk factor. Because the correlation between $\Delta c_{t}$ and $x_{\lambda, t}$ is positive (i.e., $\rho_{c x 4}=0.34$ ), so is the correlation between $\Delta c_{t}$ and $\gamma_{t}$, which may be counterintuitive. However, this relationship does not violate an inverse relationship between $\ln \nu_{t}$ and $\gamma_{t}$ because $\ln \nu_{t}$ has a negative coefficient of $x_{\lambda, t}, b_{\nu 4}=$ -0.003 . In fact, the covariance between $\ln \nu_{t}$ and $\gamma_{t}$ is negative, $\operatorname{cov}_{t}\left[\ln \nu_{t}, \gamma_{t}\right]=-0.24$. This negative covariance in turn implies a positive covariance between the $\log \operatorname{SDF} m_{t}$ and $\gamma_{t}$, which is fundamental for many models to explain high equity premiums. This relation is detailed after reporting the parameters in the subjective discount rate.

\section{Subjective discount rate}

First of all, it is noticed that the signs of the coefficients in $\ln \left(1-\beta_{t}\right)$ are all opposite to those in $\ln \nu_{t}$, indicating that the subjective discount rate moves inversely with the continuation value. This movement makes sense by recalling that the wealth-consumption ratio is solved in equilibrium as $\ln \left(W_{t}^{*} / C_{t}^{e}\right)=-\ln \left(1-\beta_{t}\right)$. So, the inverse relationship between $\ln \left(1-\beta_{t}\right)$ and $\ln \nu_{t}$ is consistent with the parallel movement between the continuation value and wealth.

More precisely, increase in $x_{f, t}$ and $x_{\lambda, t}$ increases $\ln \left(1-\beta_{t}\right)$ due to $b_{\beta 3}=30.2$ and $b_{\beta 4}=0.014$, respectively. The positive relationship between subjective discount rate and risk free rate is reasonable. From a positive $b_{\beta 4}$, it is implied that the agent raises her discount rate and hence becomes less patient when she becomes more risk averse. Conversely, increase in $x_{d, t}$ decreases $\ln \left(1-\beta_{t}\right)$ due to $b_{\beta 1}=-8.66$. It is implied that the agent lowers her discount rate and hence becomes more patient when she has brighter prospects for future consumption.

Finally, we discuss implied conditional covariances between $m_{t}$ and state-dependent preferences.

$$
\begin{aligned}
\operatorname{cov}_{t}\left[m_{t+1}, \gamma_{t+1}\right] & =b_{\gamma}^{\prime}\left\{s_{x x}\left(b_{\beta}+b_{\nu}\right)-\left(s_{x x} b_{\nu}+s_{c x}\right) \gamma_{t}\right\}=1.53 \\
\operatorname{cov}_{t}\left[m_{t+1}, \ln \left(1-\beta_{t+1}\right)\right] & =b_{\beta}^{\prime}\left\{s_{x x}\left(b_{\beta}+b_{\nu}\right)-\left(s_{x x} b_{\nu}+s_{c x}\right) \gamma_{t}\right\}=10^{-5}\left(292-1.6 \gamma_{t}\right) .
\end{aligned}
$$

The conditional covariance between $m_{t+1}$ and $\gamma_{t+1}$ is positive, which is consistent with many equilibrium models in that rise in the risk aversion raises the SDF. It is constant because only the fourth element of $b_{\gamma}$ is non-zero and because the fourth element of $\left(s_{x x} b_{\nu}+s_{c x}\right)$ is zero by 
the slope equation (62) for matching the factor risk premium of $x_{t}$. On the other hand, the conditional covariance between $m_{t+1}$ and $\ln \left(1-\beta_{t+1}\right)$ depends on $\gamma_{t}$. It is positive at $\mu_{\gamma}=150$ and remains so for $\gamma_{t}<180$. Then, it is implied that the agent more likely raises her discount rate when the SDF is high. Because future cash flows are more discounted by the compound effect, real bonds are devalued with the devaluation more significant for longer-term bonds. They therefore command high risk premiums and the resulting term structure of real interest rates will be upward-sloping.

In summary, when consumption volatility is reasonable, implied parameters of $\gamma_{t}$ are large. They are mostly determined by the factor risk premium of dividend growth, which in turn is the key to determining risk premiums of short-term dividend strips. On the other hands, the parameters in $\beta_{t}$ are more closely related with (real) bonds. They are calibrated so as to explain the real term structure. In Section 4.3, we actually generate average term structures of zero-coupon bonds and equities at Solution (e) of Table 3 discussed here.

\subsection{Term structure shapes}

It is first noted that any solutions, some of which are presented in Table 3, lead to almost the same average term structures because the factor loadings $B_{n}^{i}(i=\{R, N, D\})$ do not differ among the solutions: In fact, they are always identical to those in the LW model by design of the calibration. The constant terms $A_{n}^{i}(i=\{R, N, D\})$ differ among the solutions because not all of the intercept equations are satisfied and the resulting errors have different patterns. However, given the identical loadings, these errors cannot be large by the recursive equations for $A_{n}^{i}$.

\subsubsection{Factor loadings}

We first show the term structure of factor loadings for the log-price of zero-coupon bonds and equities, $B_{n}^{i}(i=\{R, N, D\})$. The sign and shape of $B_{n}^{i}$ give us a clue of the term structure of risk premiums. Since we know the sign and magnitude of factor risk premiums, which are presented in Panel $\mathrm{C}$ of Table 4, we understand the risk premium of an asset if we know an asset-specific quantity of risks, which is associated with the loadings. Also, the knowledge about the loadings is helpful for roughly capturing the shape of the term structure of excess-return volatilities.

Figure 2 plots $B_{n}^{i}$ against $n$ (quarters). First of all, the loadings are exactly the same between the LW and proposed models, so there is virtually one curve in each graph. The plots 
are normalized by multiplying the unconditional standard deviation of each factor. Hence, they are interpreted as the loadings on the factors with unit volatility. We begin with Panel (c) showing the loadings on the risk-free-rate factor $x_{f, t}$, which is a common factor for all assets, producing the baseline risk. They are identical for all assets, negative for all $n \geq 1$, and decrease with $n$. Since the factor risk premium of $x_{f, t}$ is negative ( $-0.11 \%$ per year), the negative loadings on $x_{f, t}$ lead to positive risk premiums attributed to $x_{f, t}$ for all assets. Furthermore, the risk premiums are expected to increase with $n$ as the loadings decrease with $n$. Since for real bonds, the loadings on the other factors are zero as seen in the other panels of Figure 2, the term structure of real interest rates is expected to be upward-sloping.

Panel (b) shows that only nominal bonds have non-zero loadings on the expected-inflationgrowth factor $x_{\pi, t}$, which are negative for all $n \geq 1$ and decrease with $n$. Since the factor risk premium of $x_{\pi, t}$ is negative $\left(-0.18 \%\right.$ per year), the risk premiums attributed to $x_{\pi, t}$ become positive for all $n \geq 1$ and increase with $n$. These premiums are added to those attributed to $x_{f, t}$, and the resulting term structure of nominal interest rates will be above that of real interest rates with the difference between the two curves widening with $n$.

Panel (a) shows that only dividend strips have non-zero loadings on the expected-dividendgrowth factor $x_{d, t}$, which are positive for all $n \geq 1$ and increase with $n$. Since the factor risk premium of $x_{d, t}$ is negative $(-0.46 \%$ per year $)$, the risk premiums attributed to $x_{d, t}$ are negative for all $n \geq 1$ and more so for longer $n$. This means that a dividend strip has two opposing components of the risk premium: One is a positive risk premium attributed to $x_{f, t}$ and the other is a negative risk premium attributed to $x_{d, t}$. The latter dominates the former. Specifically, while the magnitude of the normalized loadings is similar in absolute value between $x_{d, t}$ and $x_{f, t}$ as seen in Figures 2(a) and 2(c), the normalized factor risk premium of $x_{d, t}$ is about three times larger in absolute value than that for $x_{f, t}$. Consequently, the term structure of dividend risk premiums will have a downward slope.

Finally, in Panel (d), the loadings on the price-of-risk factor $x_{\lambda, t}$ are presented, which are negative for all assets. They differ in shape, however. They decrease monotonically with $n$ for both real and nominal bonds whereas they are inversely hump-shaped for dividend strips. These shapes do not matter with the risk premium of any assets in the original LW model, however, because the factor risk premium of $x_{\lambda, t}$ is zero. They do matter with the volatility and hence the Sharp ratio. Specifically, the negative hump at around $n=35-40$ will raise the volatility of return to dividend strips with these maturities. In contrast to the LW model, 
the negative loadings on $x_{\lambda, t}$ also matter with any assets' risk premiums in the proposed model because the factor risk premium of $x_{\lambda, t}$ is negative as presented in Panel C of Table 4. Hence, the negative loadings on $x_{\lambda, t}$ generate additional risk premiums. Consequently, term structures of risk premiums in the proposed model will be above those in the LW model, which is indeed the case as seen below.

\subsubsection{Level and volatility of real and nominal interest rates}

Let $Y_{t, n}^{i}(i=\{R, N\})$ be the yield to maturity of a zero-coupon bond maturing in $n$ periods: $Y_{t, n}^{i}=-\frac{1}{n} \ln P_{t, n}^{i}$. By substituting the formulas for $P_{t, n}^{i}$ in (37) and (39),

$$
Y_{t, n}^{i}=-\frac{1}{n}\left(A_{n}^{i}+B_{n}^{i \prime} x_{t}\right) \quad(i=\{R, N\})
$$

Then, the unconditional mean and variance of $Y_{t, n}^{i}$ are $-A_{n}^{i} / n$ and $B_{n}^{i \prime} \operatorname{var}\left[x_{t}\right] B_{n}^{i} / n^{2}$, respectively. These moments, expressed in quarterly terms, are annualized by multiplying by four.

Figure $3(\mathrm{a})$ plots the annualized unconditional mean of real interest rates, $4 E\left[Y_{t, n}^{R}\right]$, against $n$ (quarters) produced by the LW (dotted line) and proposed (solid line) models. Both plots are upward-sloping. By design of the calibration in which the real risk-free rate is matched exactly between the two models, both plots start from the same point at $n=1$. By increasing $n$, they deviate gradually. At $n=160$ (forty years), the mean real interest rate for the proposed model is higher by $1 \%$ than that for the LW model in line with the argument in Section 4.3.1.

Figure 3(b) plots the annualized unconditional mean of nominal interest rates, $4 E\left[Y_{t, n}^{N}\right]$. Again, both plots are upward-sloping and above those for real interest rates due to additional risk premiums attributed to $x_{\pi, t}$. They start from almost the same point at $n=1$ and deviate gradually with the proposed model producing higher nominal rates. The deviation reaches $1.65 \%$ at $n=160$.

Figures 3(c) and 3(d) plot the annualized unconditional standard deviation of real and nominal interest rates, $\sqrt{4 \operatorname{var}\left[Y_{t, n}^{i}\right]} \quad(i=\{R, N\})$. Both plots are downward-sloping. There is no discrepancy between the LW and proposed models because the loadings $B_{n}^{i}(i=\{R, N\})$ are the same between the two models for any $n$.

Figures 4(a) and 4(b) plot the term structures of real and nominal interest rates generated by the proposed model when the risk-free-rate factor $x_{f, t}$ is above $(+2 \mathrm{SD})$ or below $(-2 \mathrm{SD})$ two standard deviations from the mean while the other factors are fixed at the mean (i.e., zero). Consistent with intuition, when $x_{f, t}$ is high (low), both real and nominal curves shift upward 
(downward) with the shift more significant at the short end.

Analogous plots are shown in Figures 4(c) and 4(d), where the price-of-risk factor $x_{\lambda, t}$ is above or below two standard deviations from the mean with the other factors fixed at the mean. It is noted that this is equivalent to changing the risk aversion coefficient $\gamma_{t}$ by plus or minus two standard deviations from the mean because $\gamma_{t}$ is a linear function of $x_{\lambda, t}$. Also consistent with intuition, when the agent becomes more risk averse (i.e., $\gamma_{t}$ is high), both curves shift upward as she requires high risk premiums. When she is less risk averse (i.e., $\gamma_{t}$ is low), both real and nominal interest rates first decrease up to $n=12-16$ (three to four years) and then increase because of the constant term, $-A_{n}^{i} / n$, which is increasing in $n$ as shown in Figures 3(a) and $3(\mathrm{~b})$.

The term structures of interest rates in Figures 4(e) and 4(f) are drawn when the economy is "Good" and "Bad," respectively. We arguably define a good (bad) state of the economy as a state with low (high) $x_{\lambda, t}$ and high (low) $\left(x_{d, t}, x_{\pi, t}, x_{f, t}\right)$. The high (low) value corresponds to two standard deviations above (below) the mean. When the economy is good, both real and nominal interest rates start from high levels, decrease with increasing $n$ up to around $n=40$ (ten years), and then turn slightly increasing. Overall, both curves can be regarded as flat- or downward-sloping. In contrast, when the economy is bad, both curves are sharply upward-sloping, starting with low levels. The nominal interest rate at $n=1$ is negative as the model consists of Gaussian state variables. These plots do not seem to deviate largely from real observations of the economy though some level adjustments may be necessary.

\subsubsection{Risk premium, volatility, and Sharpe ratio of dividend strips}

Let $r_{t+1, n-1}^{D}$ be the log return to a dividend strip, defined and developed as

$$
\begin{aligned}
r_{t+1, n-1}^{D} & =\ln \left(\frac{P_{t+1, n-1}^{D}}{P_{t, n}^{D}}\right) \\
& =\ln \left(\frac{P_{t+1, n-1}^{D} / D_{t+1}}{P_{t, n}^{D} / D_{t}}\right)+\ln \left(\frac{D_{t+1}}{D_{t}}\right) \\
& =\left(\sigma_{x} B_{n-1}^{D}+\sigma_{d}\right)^{\prime} z_{t+1}+\operatorname{res}_{t}^{D},
\end{aligned}
$$

where $\operatorname{res}_{t}^{D}$ collects the remaining terms observed at time $t$. We define the risk premium of a dividend strip, denoted as $R P_{t, n-1}^{D}$, based on the excess log return adjusted for convexity or Jensen's inequality term:

$$
R P_{t, n-1}^{D}=E_{t}\left[r_{t+1, n-1}^{D}\right]-r_{f, t+1}+\frac{1}{2} \operatorname{var}_{t}\left[r_{t+1, n-1}^{D}\right]
$$




$$
=-\operatorname{cov}_{t}\left[m_{t+1}, r_{t+1, n-1}^{D}\right]
$$

where the second equality follows from the Euler equation, $E_{t}\left[e^{m_{t+1}+r_{t+1, n-1}^{D}}\right]=1$. By developing the conditional covariance in (71),

$$
R P_{t, n-1}^{D}=A_{n-1}^{R P D}+B_{n-1}^{R P D} \gamma_{t}
$$

where

$$
\begin{aligned}
& A_{n-1}^{R P D}=-\left(b_{\beta}+b_{\nu}\right)^{\prime}\left(s_{x x} B_{n-1}^{D}+s_{d x}\right), \\
& B_{n-1}^{R P D}=s_{d x}^{\prime} b_{\nu}+s_{c d}+\left(s_{x x} b_{\nu}+s_{c x}\right)^{\prime} B_{n-1}^{D} .
\end{aligned}
$$

The unconditional risk premium is then obtained as $E\left[R P_{t, n-1}^{D}\right]=A_{n-1}^{R P D}+B_{n-1}^{R P D} \mu_{\gamma}$.

Figure 5(a) plots the annualized unconditional mean of risk premiums, $4 E\left[R P_{t, n-1}^{D}\right]$, against $n$ (quarters) implied by the LW (dotted line) and proposed (solid line) models. Both plots are downward-sloping. They start from the same point, 17\%, which is remarkably high. By increasing $n$, they deviate gradually with the proposed model again producing higher risk premiums. At $n=160$, the risk premiums implied by the LW and proposed models are, respectively, $4.4 \%$ and $5.8 \%$, and both curves are almost flat.

Next, we compute the unconditional variance of excess return to a dividend strip, $\operatorname{var}\left[r_{t+1, n-1}^{D-}\right.$ $\left.r_{f, t+1}\right]$. First, it can be decomposed as

$$
\operatorname{var}\left[r_{t+1, n-1}^{D}-r_{f, t+1}\right]=\operatorname{var}\left[E_{t}\left[r_{t+1, n-1}^{D}-r_{f, t+1}\right]\right]+E\left[\operatorname{var}_{t}\left[r_{t+1, n-1}^{D}-r_{f, t+1}\right]\right]
$$

The first term on the RHS of (75) is developed as

$$
\operatorname{var}\left[E_{t}\left[r_{t+1, n-1}^{D}-r_{f, t+1}\right]\right]=\operatorname{var}\left[R P_{t, n-1}^{D}\right]=B_{n-1}^{R P}{ }^{\prime} \operatorname{var}\left[\gamma_{t}\right] B_{n-1}^{R P}
$$

The first equality in (76) follows from the definition of the risk premium given in (71), where $\operatorname{var}_{t}\left[r_{t+1, n-1}^{D}\right]$ is actually constant. The second equality follows by substituting (72). By (14), $\operatorname{var}\left[\gamma_{t}\right]=b_{\gamma}^{\prime} \operatorname{var}\left[x_{t}\right] b_{\gamma}$. The second term on the RHS of (75) is developed as

$$
E\left[\operatorname{var}_{t}\left[r_{t+1, n-1}^{D}-r_{f, t+1}\right]\right]=\operatorname{var}_{t}\left[r_{t+1, n-1}^{D}\right]=B_{n-1}^{D \prime} s_{x x} B_{n-1}^{D}+2 s_{d x}^{\prime} B_{n-1}^{D}+s_{d d},
$$

where the first equality follows because $r_{f, t+1}$ is observed at time $t$ and because $\operatorname{var}_{t}\left[r_{t+1, n-1}^{D}\right]$ is constant. The second equality follows by substituting (70).

Figure 5(b) plots the annualized volatility, $\sqrt{4 \operatorname{var}\left[r_{t+1, n-1}^{D}-r_{f, t+1}\right]}$, implied by the LW and proposed models. Both plots are the same because both $B_{n}^{D}$ and $B_{n}^{R P D}$ are the same for any 
$n \geq 1$ between the two models. The reason for the same $B_{n}^{D}$ is that all slope equations (59)-(62) are satisfied. In addition, $B_{n}^{R P D}$ is the same because the intercept equation (60) associated with the factor risk premium of dividend growth is satisfied. The volatility curve is hump-shaped with the peak at around $n=35-40$, which corresponds to the trough of the term structure of loadings on $x_{\lambda, t}$ shown in Figure 2(d).

Finally, we compute the unconditional Sharpe ratio of dividend strips as a ratio of the unconditional mean of risk premiums to the unconditional volatility of excess returns. Figure 5 (c) plots the annualized ratio, $4 E\left[R P_{t, n-1}^{D}\right] / \sqrt{4 \operatorname{var}\left[r_{t+1, n-1}^{D}-r_{f, t+1}\right]}$. Both plots are sharply downward-sloping with the curve for the proposed model less steep. As seen in Figures 5(a) and 5 (b), the risk premiums are high while the volatilities are low at the short end. This combination produces high Sharpe ratios. In the medium maturity range, the risk premiums decrease while the volatilities increase, leading to a sharp decrease in the Sharpe ratio. At the long end, since both risk-premium and volatility curves are almost flat, so is the curve of the Sharpe ratio.

Figure 6 presents the term structures of dividend risk premiums and Sharpe ratios generated by the proposed model when the price-of-risk factor $x_{\lambda, t}$ is above or below two standard deviations from the mean. It is noted that since risk premiums are driven by $\gamma_{t}$, which is a linear function of $x_{\lambda, t}$ alone, changing the other factors does not change the plots. Also, since excess-return volatilities are constant for any maturities, the volatility curve does not change by changing factor values. Accordingly, it is not surprising that only a parallel shift is observed in both risk-premium and Sharpe-ratio curves. Consistent with intuition, the curves shift upward (downward) when $x_{\lambda, t}$ and thus $\gamma_{t}$ are large (small).

If we wish to see a more flexible shift in the risk premium curve, such that the curve is sloped upward in times of good economy, it may be necessary to incorporate stochastic volatility into cash flow processes.

In summary, the proposed model can produce average term structures of zero-coupon bonds and equities that are close to those produced by the LW model. In the next subsection, we further examine whether the proposed model can also generate the term structure of real interest rates that is flat- or downward-sloping without much affecting the shape of the other term structures.

\subsection{Changing the shape of the real term structure}

The LW model can easily change the shape of the average term structure of real interest rates. This ability is rooted in the SDF that is driven by the same innovation term as driving dividend 
growth, which makes it easy to change the correlations between the SDF and factors affecting the real term structure. Since the proposed model does not have such a simple mechanism, it cannot change the average real yield curve as easily as the LW model. However, as demonstrated in Sections 4.1-4.3, the proposed model can replicate the LW model, which motivates us to take the following two steps to generate a downward-sloping term structure of real interest rates: The first step is to generate it using the LW model, and the second step is to replicate the LW model using the proposed model.

In the first step, we change the correlation between innovations in dividend growth $\Delta d_{t}$ and risk-free-rate factor $x_{f, t}$, denoted as $\rho_{d x 3}$, from -0.3 to 0.1 while keeping the other parameters unchanged in the LW model. When $\rho_{d x 3}=0.1$, the factor risk premium of $x_{f, t}$ is now positive because a positive shock to $x_{f, t}$ more likely increases $\Delta d_{t}$ and hence decreases the SDF. Meanwhile, it is the robust feature, regardless of the value of the correlation parameter, that the (log) price of real bonds is negatively exposed to $x_{f, t}$ and that the negative exposure is increasing with maturity. Then, the combination of a positive factor risk premium and increasingly negative exposures associated with $x_{f, t}$ results in increasingly negative risk premiums of real bonds. The term structure of real interest rates will then have a negative slope. If we wish to lower the slope further, we simply increase the value of $\rho_{d x 3}$ (up to one). But it is noted that since the loadings on $x_{f, t}$ are the same for all assets as shown in Figure 2(c), the slope for nominal interest rates and dividend risk premiums is also lowered for a more negative $\rho_{d x 3}$.

In the second step, this exogenous mechanism through correlation parameters is again endogenized by the proposed model using the parameters of consumption dynamics and statedependent preferences. They need to be re-calibrated entirely even though only a single parameter is changed in the original LW model. As in the baseline calibration, there are numerous solutions to the set of constraint equations presented in Section 3.2. We then pick a solution with $\mu_{\gamma}=150$ to ease comparison to the baseline calibration.

In Figure 7, the average term structures generated by the LW (dotted line) and proposed (solid line) models are presented: Those of volatilities are not shown for saving space. It is noted that each term structure starts from the same point as that in the baseline calibration presented in Figures 3 and 5. First, Figure 7(a) shows that the real term structure is indeed slightly downward-sloping. The proposed model generates higher rates with the deviation reaching $0.55 \%$ at $n=160$ (forty years). Second, Figure 7(b) shows that the term structure of nominal interest rates is flattened for both models, reflecting a downward-sloping real term structure. 
The nominal term structure is still positively sloped because of positive risk premiums attributed to $x_{\pi, t}$. By increasing these risk premiums, it is possible to raise the slope of the nominal term structure. In the LW model, this is achieved by making the correlation between dividend growth and realized/expected inflation growth more negative (up to minus one). In addition to this correlation channel, the proposed model has an alternative channel through state-dependent preferences, which may be beneficial when empirical evidence of the correlation channel is weak. The benefit of this alternative channel is illustrated in Section 4.5. Third, in Figures 7(c) and $7(\mathrm{~d})$, the average term structures of dividend risk premiums and Sharpe ratios remain sharply downward-sloping. In fact, the downward slope is reinforced due to negative risk premiums attributed to $x_{f, t}$.

Table 5 presents calibrated parameters for $\rho_{d x}=0.1$. There are two major changes in consumption dynamics shown in Panel A. First, the consumption-growth volatility $\sqrt{s_{c c}}$ is increased from $3.91 \%$ to $4.96 \%$, which can also be explained using the slope equation (64) for matching the factor risk premium of $\Delta d_{t}$ : In this equation, $s_{d x}^{\prime} b_{\nu}=\operatorname{cov}_{t}\left[\Delta d_{t+1}, \ln \nu_{t+1}\right]$ becomes more negative than in the baseline calibration because increase in $x_{f, t}$, which decreases the log continuation value $\ln \nu_{t+1}$ in both the previous and current calibrations due to $b_{\nu 3}<0$, now tends to increase, rather than decrease, $\Delta d_{t+1}$ by changing $\rho_{d x 3}$ from -0.3 to 0.1 . Second, the correlation between innovations in $\Delta c_{t}$ and $x_{f, t}$ (i.e., $\rho_{c x 3}$ ) is increased from -0.046 to 0.085 consistently with the change in $\rho_{d x 3}$ from -0.3 to 0.1 .

Panel B of Table 4 presents preference parameters. Overall, the signs of the parameters do not change from those in the baseline calibration. Since we originally select a particular solution with $\mu_{\gamma}=150$, an implied value of $b_{\gamma 4}$ (the coefficient of $x_{\lambda, t}$ in $\gamma_{t}$ ) does not change much from that in the baseline calibration. Instead, implied parameters in $\ln \left(1-\beta_{t}\right)$ exhibit some changes. Specifically, the unconditional mean of $\beta_{t}$ is increased from 0.987 to 0.998 , which is mainly due to the decrease of $\mu_{\beta}$ from -4.39 to -6.34 . Furthermore, implied values of $b_{\beta}$ are changed toward reducing the conditional covariance between $\ln \left(1-\beta_{t}\right)$ and $m_{t}$. Specifically, $\operatorname{cov}_{t}\left[m_{t+1}, \ln \left(1-\beta_{t+1}\right)\right]=10^{-5}\left(171-11.5 \gamma_{t}\right)$, which is now negative at $\mu_{\gamma}=150$, indicating that the agent tends to lower her discount rate when the SDF is high. The decrease of this covariance is mainly attributed to the decrease of $b_{\beta 1}$ from -8.66 to -12 . Then, a positive shock to $x_{d, t}$, which increases $m_{t}$ as reflected into a negative factor risk premium of $x_{d, t}$, decreases $\ln \left(1-\beta_{t}\right)$ more than previously. Also, the increase of $b_{\beta 3}$ from 30 to 45 has an additional contribution to decreasing this covariance. Specifically, a positive shock to $x_{f, t}$ increases $\ln \left(1-\beta_{t}\right)$ more than 
previously while it more likely decreases the SDF due to $\rho_{d x 3}=0.1$.

In summary, the proposed model is shown to be as flexible as the LW model in terms of generating the average term structure of real interest rates. Again, state-dependent time preference has an important role for this flexibility, which is crucial for any models as the shape of the real term structure is indecisive.

\subsection{Raising the slope of the nominal term structure}

As seen in Section 4.4, the real term structure is sloped downward by setting $\rho_{d x 3}=0.1$. But at the same time, the nominal term structure is flattened. We attempt to raise the nominal slope while keeping the real slope negative by changing not correlation parameters but some preference parameters.

There are two approaches for this purpose. The first is a direct approach, which is to increase $b_{\gamma 2}$ (the coefficient of $x_{\pi, t}$ in $\gamma_{t}$ ), originally set at zero. Specifically, we set $b_{\gamma 2}=90$. Then, the agent dislikes increase in $x_{\pi, t}$ more than previously as it increases the risk aversion $\gamma_{t}$ and thus the $\log \mathrm{SDF} m_{t}$. Consequently, she requires higher risk premiums of holding nominal bonds that are exposed (increasingly) negatively to $x_{\pi, t}$, and the term structure of nominal interest rates will be more positively sloped. It is noted that after changing the value of $b_{\gamma 2}$, we need to solve the recursive equation (8) for the continuation value $\nu_{t}$. Then, the solution would not exist if $b_{\gamma 2}$ were changed largely from zero (the original value) because the rest of the parameters regarding consumption dynamics and state-dependent preferences remain unchanged.

Figures $8(\mathrm{a})$ and $8(\mathrm{~b})$ plots the average term structures of real and nominal interest rates for $b_{\gamma 2}=90$ together with those for $b_{\gamma 2}=0$ (the same plots shown in Figures $7(\mathrm{a})$ and $7(\mathrm{~b})$ ). Indeed, the nominal term structure is shifted upward with the shift more significant at the long end. Consequently, the spread between nominal interest rates at $n=160$ and $n=1$ is increased from $1.44 \%$ for $b_{\gamma 2}=0$ to $1.79 \%$ for $b_{\gamma 2}=90$.

The second is an indirect approach, which is to increase $b_{\beta 2}$ (the coefficient of $x_{\pi, t}$ in $\ln (1-$ $\left.\beta_{t}\right)$ ), originally set at zero. Specifically, we set $b_{\beta 2}=2$. The reason that a positive $b_{\beta 2}$ raises the slope of the nominal term structure is less obvious but will be understood by recalling an inverse relationship between $\ln \left(1-\beta_{t}\right)$ and $\ln \nu_{t}$ as evidenced in Tables 4 and 5 . Then, it is not surprising that by setting $b_{\beta 2}>0$, the corresponding coefficient in $\ln \nu_{t}, b_{\nu 2}$, which is obtained as a solution to the recursive equation (8), is negative. By a negative $b_{\nu 2}$, the agent becomes more risk averse to increase in $x_{\pi, t}$ as it reduces the continuation value. It is also noted that as 
is the case for $b_{\gamma 2}$, if $b_{\beta 2}$ were changed largely from zero (the original value), there would be no solution for $\nu_{t}$.

Figures $8(\mathrm{c})$ and $8(\mathrm{~d})$ plots the average term structures of real and nominal interest rates for $b_{\beta 2}=2$ together with those for $b_{\beta 2}=0$ (the same plots shown in Figures $7(\mathrm{a})$ and $7(\mathrm{~b})$ ). Again, the nominal term structure is more upward-sloping with the spread between long-term and short-term rates increased to $2.14 \%$.

In summary, the proposed model can raise the slope of the term structure of nominal interest rates when the term structure of real interest rates is sloped downward and the correlation between consumption and inflation growth is moderate. The key is again state-dependent preference parameters, which control for agent's aversion to inflation risks.

\section{Alternative parameter values and cash flow dynamics}

While the proposed equilibrium model can generate the term structures of bonds and equities as flexibly as the reduced-form LW model, it obtains some counterfactual implications about consumption dynamics and/or preferences. The purpose of this section is to make the proposed model plausible from an economic point of view. There are two steps to achieve this purpose. First in Section 5.1, we change some parameter values originally calibrated by LW (2011) and then re-calibrate the parameters of the proposed model in the same procedure as explained in Section 3.2. This change is aimed at reducing the mean and standard deviation of the statedependent risk aversion without much increasing the volatility of consumption growth. Second in Section 5.2, we slightly deviate from the LW model and incorporate jumps into cash flows to further reduce the consumption volatility.

\subsection{Changing some parameter values of the LW model and re-calibrating the proposed model}

The values of the following three parameters originally calibrated by LW (2011) are changed while the other parameters are kept fixed at the values presented in Table 2:

$$
\begin{array}{lrll}
\sqrt{\operatorname{var}_{t}\left[x_{\lambda, t+1}\right]}: & 4 & \rightarrow & 0.2, \\
\sqrt{\operatorname{var}_{t}\left[\Delta d_{t+1}\right]}: & 10 \% & \rightarrow & 18 \% \text { (per year) }, \\
E\left[x_{\lambda, t}\right]: & 17 & \rightarrow & 2.62\left(=0.085 / 0.18^{2}\right),
\end{array}
$$


where $x_{\lambda, t}$ is a price-of-risk factor and $\Delta d_{t}$ is realized dividend growth. The reduction of the volatility of $x_{\lambda, t}$ from 4 to 0.2 is aimed at reducing the variance of the state-dependent risk aversion, which is specified as $\gamma_{t}=\mu_{\gamma}+b_{\gamma 4}\left(x_{\lambda, t}-\mu_{\lambda}\right)$. This change, however, also reduces return volatility for dividend strips, shifting downward the volatility term structure with the shift more significant at the short end. To offset the downward shift, the volatility of innovation in $\Delta d_{t}$, simply denoted as $\sqrt{s_{d d}}$, is increased from $10 \%$ to $18 \%$ per year, which seems to be still in an acceptable range. In the LW model, the increase in $s_{d d}$ directly raises the factor risk premium of $\Delta d_{t}$, or equivalently the risk premium of the one-period dividend strip, as it is given by $E\left[-\operatorname{cov}_{t}\left[m_{t+1}^{L W}, \Delta d_{t+1}\right]\right]=s_{d d} E\left[x_{\lambda, t}\right]$. To keep it in a reasonable range, then, $E\left[x_{\lambda, t}\right]=\mu_{\lambda}$ is reduced from 17 to 2.62 . It is note that by this change, we lower the factor risk premium of $\Delta d_{t}$ by half, from $17 \%$ to $8.5 \%$ per year. Still, the level of $8.5 \%$ seems to stand as a challenge to equilibrium models; see Table 1.

After changing the values of the three parameters in the LW model as above, the parameters of the proposed model are calibrated by the same procedure as explained in Section 3.2. Again, there are numerous solutions to the set of constraint equations, and among them we focus on the solution with $E\left[\gamma_{t}\right]=\mu_{\gamma}=30$. This is because we wish to highlight the degree to which the volatility of consumption growth decreases when the mean risk aversion is fixed at 30 . The level of 30 may still be large but is in a range of values considered or estimated by the previous work: 21 (Bansal and Shaliastovich, 2013), 50 (Doh and Wu, 2016), 66 (van Binsbergen, FernandezVillaverde, Koijen, and Rubio-Ramirez, 2012), and 75 (Rudebusch and Swanson, 2012).

The results of the calibration are provided in the "No JUMP" row of Table 6. The total volatility (Total vol.) is computed as $\sqrt{\operatorname{var}_{t}\left[\Delta c_{t+1}\right]}$ for consumption growth and $\sqrt{\operatorname{var}_{t}\left[\Delta d_{t+1}\right]}$ for dividend growth. They are the same as $\sqrt{s_{c c}}\left(=\sqrt{\sigma_{c}^{\prime} \sigma_{c}}\right)$ and $\sqrt{s_{d d}}\left(=\sqrt{\sigma_{d}^{\prime} \sigma_{d}}\right)$, respectively, in the current model: In Section 5.2, we extend the model by introducing jumps into cash flows, and in this extended model, $\sqrt{s_{c c}}$ and $\sqrt{s_{d d}}$ correspond to the Gaussian component of the total volatility.

An implied $\sqrt{s_{c c}}$ is $5.05 \%$ per year, which is smaller than $8.84 \%$ in the baseline calibration presented in Table 3 but still seems to be large relative to historical estimates. An intuitive explanation of why the volatility of consumption growth is smaller in spite of the fact that the volatility of dividend growth is increased from $10 \%$ to $18 \%$ is as follows. By reducing the volatility of $x_{\lambda, t}, b_{\gamma 4}$ (the coefficient of $x_{\lambda, t}$ in $\gamma_{t}$ ) is increased from 1.78 in the baseline calibration (i.e., Solution (a) of Table 3) to 12.03: These numbers are not shown in any tables. 
The result indicates that though both $b_{\gamma 4}$ and $s_{d d}$ are increased, the rate of increase is larger in the former than in the latter. It then follows from the slope equation for the factor risk premium of $\Delta d_{t}$, given by $b_{\gamma 4}\left(s_{d x}^{\prime} b_{\nu}+s_{c d}\right)=s_{d d}$, that the consumption volatility, which appears in $s_{c d}=\sqrt{s_{c c} s_{d d}} \rho_{c d}$, does not need to be as large as in the baseline calibration.

The unconditional standard deviation of $\gamma_{t}$, denoted as $S D\left[\gamma_{t}\right]$, is implied to be 8.6, which is smaller than 25 shown in Table 3. Panel (a) of Figure 9 depicts the unconditional distribution of $\gamma_{t}$. Though still non-zero due to the normal distribution, the probability of $\gamma_{t}<0$ is negligibly small, showing that one of the shortcomings of the proposed model is resolved.

The unconditional mean and standard deviation of the subjective discount factor, denoted as $E\left[\beta_{t}\right]$ and $S D\left[\beta_{t}\right]$, are 0.996 and 0.00085 , respectively. Compared with the corresponding values at row (a) of Table 3, the mean becomes more reasonable, and the standard deviation becomes much smaller because of a lower volatility of $x_{\lambda, t}$. As noted in Sections 2.6 and 4.1, a lower volatility of $\beta_{t}$ improves the accuracy of the approximation to the continuation value $\nu_{t}$. Panel (b) of Figure 9 depicts the unconditional distribution of $\beta_{t}$, showing that the peak is near the upper bound of one and that the left tail is not long.

Figure 10 presents the average term structures of real and nominal interest rates. Panels (a) and (b) show that both yield curves are upward-sloping. The slope, however, is less steep than in the baseline calibration shown in Figure 3 because of a smaller mean of $x_{\lambda, t}$. Panels (c) and (d) show that both volatility curves are downward-sloping. The negative slope is more pronounced than in the baseline calibration because of a lower volatility of $x_{\lambda, t}$.

Figure 11 presents the term structures of risk premiums, excess-return volatilities, and Sharpe ratios of dividend strips. Panel (a) shows that the proposed model can still generate a downwardsloping term structure of dividend risk premiums. The risk premiums at $n=1$ (one quarter) and $n=160$ (forty years) are $8.50 \%$ and $5.77 \%$ per year, respectively. Though the range is narrower than in the baseline calibration, it is still comparable to those for the previous models listed in Table 1. Panel (b) shows that the volatility curve is first decreasing up to around $n=40$ (ten years) and then turns slightly increasing. The volatilities at $n=1,40$, and 160 are, respectively, $18.0 \%, 15.2 \%$, and $16.3 \%$ per year. Compared with the baseline calibration shown in Figure 5, the volatilities at the short end are higher, reflecting the increase in the volatility of $\Delta d_{t}$. In the medium maturity range, they are lower due to a lower volatility of $x_{\lambda, t}$. At the long end, the volatilities in both the baseline and alternative calibrations converge to a similar level. Panel (c) shows that the model can also generate a downward-sloping term structure of Sharpe ratios, 
ranging from $0.47(n=1)$ to $0.35(n=160)$. The range seems to be comparable to the previous models though narrower than in the baseline calibration.

In summary, the proposed model can still generate the term structures that stand as challenges to equilibrium models. But changing parameter values alone may not be sufficient because the volatility of $\Delta c_{t}$ still seems to be high. In Section 5.2, we modify the dynamics of cash flows to overcome this problem. But the modification is kept minimal as our focus is on the extension of preference, or discount, rather than cash flows.

\subsection{Introducing jumps into cash flows}

To further reduce the volatility of consumption growth, we introduce jumps into both consumption and dividend processes. The jumps can be interpreted as disastrous events in line with Reitz (1988), Barro (2009), Gabaix (2012), and Wachter (2013). The agent dislikes jump shocks (infrequent but large negative shocks) to consumption growth more than Gaussian shocks (small but frequent shocks) if these two types of shocks have the same volatility in a statistical sense. Then, for a given level of the agent's measure of consumption risks, it is possible to reduce a statistical measure of consumption risks by introducing jumps.

\subsubsection{Cash flow dynamics and the derived SDE}

Our introduction of jumps is simple by assuming that realized consumption and dividend growth alone can jump: Neither inflation growth nor state vector can. Additionally, we assume that both jump intensity and jump size are constant. Then, we re-specify the consumption and dividend processes as

$$
\begin{aligned}
& \Delta c_{t+1}=\mu_{c}+b_{c} x_{d, t}+\sigma_{c}^{\prime} z_{t+1}+(\ln \xi) N_{t+1} \\
& \Delta d_{t+1}=\mu_{d}+x_{d, t}+\sigma_{d}^{\prime} z_{t+1}+k(\ln \xi) N_{t+1}
\end{aligned}
$$

where $N_{t}$ follows an i.i.d. Poisson distribution with intensity parameter $l>0$ and is independent of the Gaussian shock $z_{t}$. A jump size in consumption growth is captured by $\xi(0<\xi<1)$. When a single jump occurs at time $t+1$ (i.e., $N_{t+1}=1$ ), $C_{t+1}^{e}=\xi C_{t}^{e}$, ignoring the other components. Multiple jumps at a point in time are also possible, which can be interpreted as representing how serious the disaster is. Specifically, when $N_{t+1}=n, C_{t+1}^{e}=\xi^{n} C_{t}^{e}$. But this interpretation makes it difficult to identify $l$ and $\xi$ separately as both are related to the disaster's 
seriousness. Then, we fix $l$ at $1 / 40$, which roughly corresponds to the frequency at which a jump occurs once in every ten years on average.

The same $N_{t+1}$ is used for capturing jumps in dividend growth, which means that the jump event occurs to both processes simultaneously. But the jump size for dividend growth is amplified by $k>1$ because $D_{t+1}=\xi^{n k} D_{t}$ for $N_{t+1}=n$, ignoring the other components.

It is noted that a number of extensions are possible regarding a jump component and accordingly that the results below can be further improved. First, the jump size can be stochastic. A conventional probability distribution such as an exponential, gamma, or normal distribution does not violate the model's tractability. Second, the jump intensity can be stochastic. Gabaix (2012) and Watcher (2013) demonstrate the importance of time-varying jump intensity for capturing high equity premiums. Third, the disaster can be followed by the recovery. Hasler and Marfè (2016) model consumption and dividend processes that mean-revert after a large fall, with the rate of mean-reversion differing between the two processes, and successfully explain a downward-sloping term structure of dividend risk premiums.

For notational simplicity, we denote the consumption process by the sum of Gaussian and Jump components as $\Delta c_{t+1}=\Delta c_{t+1}^{G}+\Delta c_{t+1}^{J}$, where $\Delta c_{t+1}^{J}=(\ln \xi) N_{t+1}$ and $\Delta c_{t+1}^{G}$ is the remaining Gaussian component (including the predictable one). Likewise, the dividend process is denoted as $\Delta d_{t+1}=\Delta d_{t+1}^{G}+\Delta d_{t+1}^{J}$.

The $\log$ continuation value $\ln \nu_{t}$ is also approximated by a linear function of the state vector $x_{t}$ as $\ln \nu_{t}=\mu_{\nu}+b_{\nu}^{\prime} x_{t}$, where $\left(\mu_{\nu}, b_{\nu}^{\prime}\right)$ is the solution to simultaneous quadratic equations, which are slightly modified due to the jump component. They are provided in Appendix A and the accuracy of the approximation to $\nu_{t}$ is reported in Appendix C.

The log SDF $m_{t+1}$ is derived as

$$
m_{t+1}=-r_{f, t+1}-\frac{1}{2} \lambda_{t}^{\prime} \lambda_{t}-\lambda_{t}^{\prime} z_{t+1}-\gamma_{t}(\ln \xi) N_{t+1}-l\left(\xi^{-\gamma_{t}}-1\right) .
$$

The real risk-free rate $r_{f, t+1}$ is also derived as a linear function of $x_{t}: r_{f, t+1}=A_{f}+B_{f}^{\prime} x_{t}$, where $\left(A_{f}, B_{f}^{\prime}\right)$ are also adjusted for the jump component, presented in Appendix A. The price-of-risk vector $\lambda_{t}$ associated with Gaussian shocks is of the same form as given in (32). But it is noted that it depends implicitly on jump parameters through $\left(\mu_{\nu}, b_{\nu}^{\prime}\right)$.

The $\log$ prices of zero-coupon bonds and equities are approximated as linear in $x_{t}: \ln P_{t, n}^{i}=$ $A_{n}^{i}+B_{n}^{i \prime} x_{t}(i=\{R, N, D\})$. For real and nominal bonds, the recursive equations for $\left(A_{n}^{i}, B_{n}^{i \prime}\right)$ $(i=\{R, N\})$ are of the same form as those without the jump component: They depend on jump 
parameters indirectly through $\left(\mu_{\nu}, b_{\nu}^{\prime}, A_{f}, B_{f}^{\prime}\right)$. In contrast, for dividend strips, the recursive equations for $\left(A_{n}^{D}, B_{n}^{D \prime}\right)$ have additional terms related to the jump component because their payoffs directly depend on future dividends that are exposed to jump shocks. The price of a dividend strip when jumps are included is derived in Appendix D.

\subsubsection{Calibration}

The parameters of the extended model are calibrated by taking the following conditions into account: $E\left[\gamma_{t}\right]=30 ; E\left[\Delta c_{t}\right]=E\left[\Delta d_{t}\right]=1.29 \%$ per year in equations (78) and (79) (the same level as in the baseline calibration); $\operatorname{var}_{t}\left[\Delta c_{t+1}\right]=4 \%$ per year in equation $(78) ; \operatorname{var}_{t}\left[\Delta d_{t+1}\right]=$ $18 \%$ per year in equation $(79) ; l=1 / 40$; and the average term structures of interest rates and dividend risk premiums do not change much from those in Section 5.1.

The results of the calibration are provided in the "JUMP" row of Table 6. It is first noted that the solution to (an approximation of) the continuation value and hence the SDF does exist that satisfies the conditions listed above. Indeed, we can successfully reduce the volatility of consumption growth while keeping reasonable the behavior of state-dependent preferences. Of the total volatility of $\Delta c_{t}$ set at $4 \%$, the Gaussian component $\sqrt{s_{d d}}$ reaches $3.98 \%$. Once a jump event occurs with the intensity set at $l=1 / 40$, the current consumption falls by $1.26 \%$ (computed by $\xi-1$ ) from the previous quarter. These results imply that the role of jumps is not crucial for consumption growth. It is, however, for dividend growth. Of the total volatility of $\Delta d_{t}$ set at $18 \%$, the Gaussian component is $16.52 \%$, and upon occurrence of a jump, the dividend falls by more than $20 \%$ (computed by $\xi^{k}-1$ ) from the previous quarter.

The standard deviation of the state-dependent risk aversion is decreased from 8.6 to 8.1 when jumps are included. The difference, however, does not seem to be economically large, judged from the unconditional distribution of $\gamma_{t}$ presented in Figure 9(a). Indeed, the probability of $\gamma_{t}<0$ is negligibly small in both cases.

On the other hand, the inclusion of jumps can change the mean and standard deviation of the subjective discount factor $\beta_{t}$. The unconditional mean $E\left[\beta_{t}\right]$ is decreased to 0.986 while the unconditional standard deviation $S D\left[\beta_{t}\right]$ is increased to 0.00232 . But still, the mean is reasonable and the standard deviation is smaller than in the baseline calibration because of the decrease in the volatility of $x_{\lambda, t}$. Consequently, the accuracy of the approximation to the continuation value $\nu_{t}$ is maintained even by the introduction of jumps: The results of the accuracy with jumps are provided in Appendix C. We plot the unconditional distribution of $\beta_{t}$ in Figure 9(b), 
noticing leftward shift and longer left tail. The change of the distribution can be explained as follows. Facing jump risks in consumption growth, the agent is more willing to hold real bonds, which potentially shifts the real yield curve downward. But since we do not change the level of the one-period risk-free rate from that without jumps, the subjective discount rate $1-\beta_{t}$ must increase and hence $\beta_{t}$ must decrease. Since $\beta_{t}$ is shifted away from the upper bound of one, there is more room for $\beta_{t}$ to fluctuate.

\subsubsection{Term structures}

The term structures of interest rates and their volatilities are plotted in Figure 10. By construction of the calibration procedure explained in Section 5.2.2, each plot is similar between with and without jumps. The same is true for the term structure of dividend risk premiums shown in Figure 11(a). On the other hand, a difference appears in Figure 11(b) showing the term structure of excess-return volatilities of dividend strips. By including jumps, the volatility curve in the medium to long maturity range is shifted upward while at $n=1$, the volatility is almost the same between with and without jumps because of the constraint that the volatility of dividend growth is exactly the same, set at $18 \%$. At $n=160$, excess-return volatility with jumps is $17.0 \%$, which is larger than $16.3 \%$ without jumps. An intuitive explanation of the upward shift is as follows. Among the four factors, the expected-dividend-growth factor $x_{d, t}$ dominates return volatility for medium- to long-term dividend strips. By introducing jumps into realized dividend growth but not into expected dividend growth, the covariance between realized and expected dividend growth becomes less negative than it is before introducing jumps, because the role of the Gaussian component, which matters with the covariance, is less important with the presence of jumps. The less negative covariance then leads to higher return volatility for these strips. Since the volatility is slightly higher with jumps than without, the Sharpe ratios decrease slightly faster as shown in Figure 11(c).

In summary, the change in some parameter values and the inclusion of jumps into cash flows together are helpful for improving the proposed model, which now offers economically reasonable implications about consumption dynamics and preferences while keeping the ability to generate various term structures. 


\section{Concluding remarks}

This study proposes an equilibrium asset pricing model with the purpose of jointly producing the term structures of zero-coupon bonds and equities. For this purpose, we extend a recursive utility function in a way where the parameters capturing risk aversion and time preference are driven by state variables of the economy and asset markets, enabling the agent to more directly express her preferences for cash flows at various points in time. The parameters of the proposed model are calibrated by matching the stochastic discount factor of the proposed model with that exogenously specified by Lettau and Wachter (LW) (2011). This calibration approach allows the proposed model to have a similar descriptive ability to the LW model, and the LW model to have an equilibrium foundation. With the help of the LW model, the proposed model can produce a downward-sloping term structure of dividend risk premiums when the term structure of real interest rates is sloped either upward or downward, which is considered as challenges to equilibrium models.

At the same time, we uncover that the values of the parameters originally calibrated by LW may not be realistic when more economic structures are imposed. Most notably, it is implied that the mean and variance of a state-dependent risk aversion is too high given a reasonable level of consumption growth volatility. We then change some parameter values of the LW model, which shifts some risks from a price-of-risk factor to realized dividend growth. We further introduce into consumption and dividend processes jumps, which can be interpreted as disasters. The model then implies an economically plausible behavior of both preferences and consumption growth without losing the descriptive ability for the term structures.

This study presents an approach for creating an equilibrium asset pricing model that is as flexible as a reduced-form model. But it does not address which state variables really affect agent's preferences nor how the model is estimated using macro and finance data. These, together with collecting more evidence on state-dependent preferences, are left for future research. 


\section{Appendix A: Derivation of key equations}

\section{The optimal consumption given in equation (6)}

Substitute $V_{t}$ into (2) with $C_{t}$ replaced by $C_{t}^{*}$ (the optimal consumption for the agent):

$$
V_{t}=C_{t}^{* 1-\beta_{t}} E_{t}\left[V_{t+1}^{1-\gamma_{t}}\right]^{\beta_{t} /\left(1-\gamma_{t}\right)} .
$$

Assume that $V_{t}$ is of the form $V_{t}=\phi_{t} W_{t}$, where $\phi_{t}$ is a state-dependent variable identified below. Substitute first this form and then the budget constraint given in (4) into the RHS of (81):

$$
V_{t}=C_{t}^{* 1-\beta_{t}}\left(W_{t}-C_{t}^{*}\right)^{\beta_{t}} E_{t}\left[\left(\phi_{t+1} R_{w, t+1}\right)^{1-\gamma_{t}}\right]^{\beta_{t} /\left(1-\gamma_{t}\right)} .
$$

(82) satisfies the first order condition (FOC): $\partial V_{t} / \partial C_{t}^{*}=0$. Given that $\beta_{t}$ and $\gamma_{t}$ are exogenous by Assumption (i), solving the FOC yields $C_{t}^{*}$ given in (6). By Assumption (ii), the second order condition is met: $\partial^{2} V_{t} / \partial C_{t}^{* 2}<0$. Finally, by substituting (6) into (82), $V_{t}$ is confirmed to be of the assumed form, where

$$
\phi_{t}=\left(1-\beta_{t}\right)\left\{\frac{\beta_{t}}{1-\beta_{t}} E_{t}\left[\left(\phi_{t+1} R_{w, t+1}\right)^{1-\gamma_{t}}\right]^{1 /\left(1-\gamma_{t}\right)}\right\}^{\beta_{t}} .
$$

\section{Recursive equation (8) for the value function}

Replace first $R_{w, t+1}$ with $R_{w, t+1}^{*}$ on the RHS of (83) and then substitute (7):

$$
\frac{\phi_{t}^{*}}{1-\beta_{t}}=E_{t}\left[\left(\frac{\phi_{t+1}^{*}}{1-\beta_{t+1}} \frac{C_{t+1}^{e}}{C_{t}^{e}}\right)^{1-\gamma_{t}}\right]^{\beta_{t} /\left(1-\gamma_{t}\right)},
$$

where $\phi_{t}^{*}$ is used in place of $\phi_{t}$ to emphasize the equilibrium. Meanwhile, the continuation value in equilibrium is $V_{t}^{*}=\phi_{t}^{*} W_{t}^{*}=\frac{\phi_{t}^{*}}{1-\beta_{t}} C_{t}^{e}$. Define $\nu_{t}=V_{t}^{*} / C_{t}^{e}=\frac{\phi_{t}^{*}}{1-\beta_{t}}$. Substituting this into (84) yields (8).

\section{SDF given in equation (9)}

A simple way of deriving the SDF is to use the Euler equation for the wealth (a claim to the flow of endowments). Rearrange (8):

$$
1=E_{t}\left[\left(\frac{\nu_{t+1}}{\nu_{t}^{1 / \beta_{t}}}\right)^{1-\gamma_{t}}\left(\frac{C_{t+1}^{e}}{C_{t}^{e}}\right)^{-\gamma_{t}} \frac{C_{t+1}^{e}}{C_{t}^{e}}\right] .
$$

By (7),

$$
\frac{C_{t+1}^{e}}{C_{t}^{e}}=\beta_{t} \frac{1-\beta_{t+1}}{1-\beta_{t}} R_{w, t+1}^{*} .
$$

Substitute (86) into (85) and rearrange the terms, we have $1=E_{t}\left[M_{t+1} R_{w, t+1}^{*}\right]$, where $M_{t+1}$ is given in (9). 


\section{Value function given in equations (17)-(19)}

Assume that the solution to the recursive equation (8) is of the following form: $\nu_{t}=\exp \left\{\mu_{\nu}+\right.$ $\left.b_{\nu}^{\prime} x_{t}\right\}$. Substitute this form into (8) together with $\beta_{t}=\beta$ :

$$
\begin{aligned}
\exp \left\{\mu_{\nu}+b_{\nu}^{\prime} x_{t}\right\} & =E_{t}\left[\exp \left\{\left(1-\gamma_{t}\right)\left(\mu_{\nu}+b_{\nu}^{\prime} x_{t+1}+\Delta c_{t+1}\right)\right\}\right]^{\beta /\left(1-\gamma_{t}\right)} \\
& =\exp \left\{\beta\left(E_{t}\left[\chi_{t+1}\right]-\frac{1}{2}\left(\gamma_{t}-1\right) \operatorname{var}_{t}\left[\chi_{t+1}\right]\right)\right\}
\end{aligned}
$$

where

$$
\chi_{t}=\mu_{\nu}+b_{\nu}^{\prime} x_{t}+\Delta c_{t} .
$$

Note that

$$
\begin{aligned}
E_{t}\left[\chi_{t+1}\right] & =\mu_{\nu}+\mu_{c}+\left(b_{c}+\Phi_{x} b_{\nu}\right)^{\prime} x_{t}, \\
\operatorname{var}_{t}\left[\chi_{t+1}\right] & =b_{\nu}^{\prime} s_{x x} b_{\nu}+2 s_{c x}^{\prime} b_{\nu}+s_{c c}\left(=v_{c \nu}\right) .
\end{aligned}
$$

Substitute these conditional moments and $\gamma_{t}=\mu_{\gamma}+b_{\gamma}^{\prime} x_{t}$ into the RHS of (87), and then take the log of both sides:

$$
\mu_{\nu}+b_{\nu}^{\prime} x_{t}=\beta\left\{\mu_{\nu}+\mu_{c}+\left(b_{c}+\Phi_{x} b_{\nu}\right)^{\prime} x_{t}-\frac{1}{2} v_{c \nu}\left(\mu_{\gamma}+b_{\gamma}^{\prime} x_{t}-1\right)\right\} .
$$

For the assumed form of $\nu_{t}$ to be true, (91) must hold for any $x_{t}$, leading to the simultaneous equations for $\left(\mu_{\nu}, b_{\nu}^{\prime}\right)$ given in (18) and (19).

\section{Risk-free rate given in equations $(23)-(25)$}

By substituting (17) together with $\beta_{t}=\beta$ into (9), the SDF can be rewritten using $\chi_{t+1}$ defined in $(88)$ as

$$
M_{t+1}=\beta \nu_{t}^{-\left(1-\gamma_{t}\right) / \beta} \exp \left\{\left(1-\gamma_{t}\right) \chi_{t+1}-\Delta c_{t+1}\right\}
$$

Take the conditional expectation of both sides of (92):

$$
E_{t}\left[M_{t+1}\right]=\beta \nu_{t}^{-\left(1-\gamma_{t}\right) / \beta} E_{t}\left[\exp \left\{\left(1-\gamma_{t}\right) \chi_{t+1}\right\}\right] E_{t}\left[e^{-\Delta c_{t+1}}\right] \exp \left\{\left(\gamma_{t}-1\right) \operatorname{cov}_{t}\left[\chi_{t+1}, \Delta c_{t+1}\right]\right\}
$$

Meanwhile, the recursive equation (8) can be rewritten using $\chi_{t}$ as

$$
\nu_{t}^{\left(1-\gamma_{t}\right) / \beta}=E_{t}\left[\exp \left\{\left(1-\gamma_{t}\right) \chi_{t+1}\right\}\right]
$$

Substitute (94) into the RHS of (93), develop the conditional moments, and rearrange the terms:

$$
\begin{aligned}
r_{f, t+1} & =-\ln E_{t}\left[M_{t+1}\right] \\
& =-\ln \beta+\mu_{c}-\frac{1}{2} s_{c c}-\left(s_{c x}^{\prime} b_{\nu}+s_{c c}\right)\left(\mu_{\gamma}-1\right)+\left\{b_{c}-\left(s_{c x}^{\prime} b_{\nu}+s_{c c}\right) b_{\gamma}\right\}^{\prime} x_{t} .
\end{aligned}
$$


Collecting the intercept and slope terms of (95) into $A_{f}$ and $B_{f}$, respectively, yields (24) and (25).

\section{Approximation of the value function given in equations (17) and (29)-(30)}

Substitute $\nu_{t}=\exp \left\{\mu_{\nu}+b_{\nu}^{\prime} x_{t}\right\}$ into the RHS of (8), develop the conditional expectation, and rearrange the terms:

$$
\ln (\operatorname{RHS} \text { of }(8))=\beta\left(x_{t}\right)\left\{\mu_{\nu}+\mu_{c}+\left(b_{c}+\Phi_{x} b_{\nu}\right)^{\prime} x_{t}-\frac{1}{2} v_{c \nu}\left(\mu_{\gamma}+b_{\gamma}^{\prime} x_{t}-1\right)\right\},
$$

which is basically the same as the RHS of (91) except that $\beta$ is replaced by $\beta\left(x_{t}\right)$. Since (96) is not equal to $\mu_{\nu}+b_{\nu}^{\prime} x_{t}$ (the log of the LHS of (8)) for any $x_{t}$, it is approximated as linear in $x_{t}$. Specifically, nonlinear terms associated with $\beta\left(x_{t}\right)$ and $\beta\left(x_{t}\right) x_{t}$ are linearized around $x_{t}=0$ (the unconditional mean) as given in (27) and (28), respectively. Then, matching the intercept and slope terms yields (29) and (30).

Approximation of the risk-free rate given in equations (23) and (34)-(35)

By substituting (17) into (9), the SDF can be rewritten as

$$
M_{t+1}=\frac{\beta_{t}}{1-\beta_{t}} \nu_{t}^{-\left(1-\gamma_{t}\right) / \beta_{t}} \exp \left\{\left(1-\gamma_{t}\right) \chi_{t+1}+\psi_{t+1}\right\},
$$

where $\chi_{t+1}$ is defined in (88) and

$$
\psi_{t}=\mu_{\beta}+b_{\beta}^{\prime} x_{t}-\Delta c_{t} .
$$

Take the conditional expectation of both sides of (97):

$$
\begin{aligned}
& E_{t}\left[M_{t+1}\right]=\frac{\beta_{t}}{1-\beta_{t}} \nu_{t}^{-\left(1-\gamma_{t}\right) / \beta_{t}} \\
& \quad \times E_{t}\left[\exp \left\{\left(1-\gamma_{t}\right) \chi_{t+1}\right\}\right] E_{t}\left[e^{\psi_{t+1}}\right] \exp \left\{\left(1-\gamma_{t}\right) \operatorname{cov}_{t}\left[\chi_{t+1}, \psi_{t+1}\right]\right\} .
\end{aligned}
$$

Note that the recursive equation (8) can also be rewritten as $\nu_{t}^{\left(1-\gamma_{t}\right) / \beta_{t}}=E_{t}\left[\exp \left\{\left(1-\gamma_{t}\right) \chi_{t+1}\right\}\right]$. Substitute this into the RHS of (99) and develop the conditional expectation:

$$
E_{t}\left[M_{t+1}\right]=\frac{\beta_{t}}{1-\beta_{t}} \exp \left\{E_{t}\left[\psi_{t+1}\right]+\frac{1}{2} \operatorname{var}_{t}\left[\psi_{t+1}\right]+\left(1-\gamma_{t}\right) \operatorname{cov}_{t}\left[\chi_{t+1}, \psi_{t+1}\right]\right\} .
$$

Note that

$$
\begin{aligned}
E_{t}\left[\psi_{t+1}\right] & =\mu_{\beta}-\mu_{c}+\left(\Phi_{x} b_{\beta}-b_{c}\right)^{\prime} x_{t}, \\
\operatorname{var}_{t}\left[\psi_{t+1}\right] & =b_{\beta}^{\prime} s_{x x} b_{\beta}-2 s_{c x}^{\prime} b_{\beta}+s_{c c}\left(=v_{c \beta}\right), \\
\operatorname{cov}_{t}\left[\chi_{t+1}, \psi_{t+1}\right] & =\left(s_{x x} b_{\nu}+s_{c x}\right)^{\prime} b_{\beta}-\left(s_{c x}^{\prime} b_{\nu}+s_{c c}\right) .
\end{aligned}
$$


Substitute these conditional moments together with (14) and (16) into the RHS of (100), and rearrange the terms:

$$
\begin{aligned}
r_{f, t+1}= & -\ln \beta_{t}+\mu_{c}-\frac{1}{2} v_{c \beta}+\left\{\left(s_{x x} b_{\nu}+s_{c x}\right)^{\prime} b_{\beta}-\left(s_{c x}^{\prime} b_{\nu}+s_{c c}\right)\right\}\left(\mu_{\gamma}-1\right) \\
& +\left[b_{c}+\left(I_{d \times d}-\Phi_{x}\right) b_{\beta}+\left\{\left(s_{x x} b_{\nu}+s_{c x}\right)^{\prime} b_{\beta}-\left(s_{c x}^{\prime} b_{\nu}+s_{c c}\right)\right\} b_{\gamma}\right]^{\prime} x_{t} .
\end{aligned}
$$

The leading term on the RHS of $(104),-\ln \beta_{t}$, is nonlinear in $x_{t}$. It is then linearized around $x_{t}=0$ (the unconditional mean) as given in (33). Then, collecting the intercept and slope terms into $A_{f}$ and $B_{f}$, respectively, yields (34) and (35).

\section{Risk premium of a dividend strip given in equations (72)-(74)}

Note that

$$
\begin{aligned}
m_{t+1}-E_{t}\left[m_{t+1}\right] & =-\lambda_{t}^{\prime} z_{t+1}, \\
r_{t+1, n-1}^{D}-E_{t}\left[r_{t+1, n-1}^{D}\right] & =\left(\sigma_{x} B_{n-1}^{D}+\sigma_{d}\right)^{\prime} z_{t+1},
\end{aligned}
$$

where $\lambda_{t}=\left(\sigma_{x} b_{\nu}+\sigma_{c}\right) \gamma_{t}-\sigma_{x}\left(b_{\beta}+b_{\nu}\right)$. Substitute (105) and (106) into the RHS of (71),

$$
\begin{aligned}
R P_{t, n-1}^{D} & =\left\{\left(\sigma_{x} b_{\nu}+\sigma_{c}\right) \gamma_{t}-\sigma_{x}\left(b_{\beta}+b_{\nu}\right)\right\}^{\prime}\left(\sigma_{x} B_{n-1}^{D}+\sigma_{d}\right) \\
& =-\left(b_{\beta}+b_{\nu}\right)^{\prime}\left(s_{x x} B_{n-1}^{D}+s_{d x}\right)+\left\{s_{d x}^{\prime} b_{\nu}+s_{c d}+\left(s_{x x} b_{\nu}+s_{c x}\right)^{\prime} B_{n-1}^{D}\right\} \gamma_{t}
\end{aligned}
$$

\section{Approximation of the value function with jumps in consumption growth}

By $\Delta c_{t}=\ln \left(C_{t}^{e} / C_{t-1}^{e}\right)=\Delta c_{t}^{G}+\Delta c_{t}^{J}$, the recursive equation for the log continuation value can be written as

$$
\ln \nu_{t}=\frac{\beta_{t}}{1-\gamma_{t}} \ln E_{t}\left[\exp \left\{\left(1-\gamma_{t}\right)\left(\ln \nu_{t+1}+\Delta c_{t+1}^{G}+\Delta c_{t+1}^{J}\right)\right\}\right] .
$$

Because $\Delta c_{t}^{G}$ and $\Delta c_{t}^{J}$ are mutually independent and because $\nu_{t}$ is a function of the state vector that has no jump component, the RHS of (108) can be factorized as follows:

$$
\ln \nu_{t}=\frac{\beta_{t}}{1-\gamma_{t}}\left(\ln E_{t}\left[\exp \left\{\left(1-\gamma_{t}\right)\left(\ln \nu_{t+1}+\Delta c_{t+1}^{G}\right)\right\}\right]+\ln E_{t}\left[\exp \left\{\left(1-\gamma_{t}\right) \Delta c_{t+1}^{J}\right\}\right]\right) .
$$

For $\Delta c_{t+1}^{J}=(\ln \xi) N_{t+1}$, the second term on the RHS of (109) is developed as

$$
\frac{\beta_{t}}{1-\gamma_{t}} \ln E_{t}\left[\exp \left\{\left(1-\gamma_{t}\right)(\ln \xi) N_{t+1}\right\}\right]=\frac{l \beta_{t}\left(\xi^{1-\gamma_{t}}-1\right)}{1-\gamma_{t}} .
$$

To approximate $\ln \nu_{t}$ as $\ln \nu_{t}=\mu_{\nu}+b_{\nu}^{\prime} x_{t}$, it is necessary to linearize the RHS of (110) as,

$$
\frac{l \beta_{t}\left(\xi^{1-\gamma_{t}}-1\right)}{1-\gamma_{t}} \approx-\left(1-e^{\mu_{\beta}}\right) l k_{0}+l k_{1}^{\prime} x_{t}
$$


where

$$
\begin{aligned}
& k_{0}=\frac{\xi^{1-\mu_{\gamma}}-1}{\mu_{\gamma}-1} \\
& k_{1}=e^{\mu_{\beta}} k_{0} b_{\beta}+\frac{1-e^{\mu_{\beta}}}{\mu_{\gamma}-1}\left\{k_{0}+\xi^{1-\mu_{\gamma}} \ln \xi\right\} b_{\gamma} .
\end{aligned}
$$

It is noted that this approximation is unavoidable even for $\beta_{t}=\beta$ (constant). Then, it is concerned that the approximation to $\nu_{t}$ is less accurate, which is addressed in Appendix C.

By the additional approximation given in $(111),\left(\mu_{\nu}, b_{\nu}^{\prime}\right)$ satisfies the following equations:

$$
\begin{aligned}
& \mu_{\nu}=\left(1-e^{\mu_{\beta}}\right)\left\{\mu_{\nu}+\mu_{c}-\frac{1}{2} v_{c \nu}\left(\mu_{\gamma}-1\right)-l k_{0}\right\} \\
& b_{\nu}=\left(1-e^{\mu_{\beta}}\right)\left(b_{c}+\Phi_{x} b_{\nu}-\frac{1}{2} v_{c \nu} b_{\gamma}\right)-e^{\mu_{\beta}}\left\{\mu_{\nu}+\mu_{c}-\frac{1}{2} v_{c \nu}\left(\mu_{\gamma}-1\right)\right\} b_{\beta}+l k_{1} .
\end{aligned}
$$

\section{Risk-free rate with jumps in consumption growth}

We approximate the risk-free rate $r_{f, t+1}$ as $r_{f, t+1}=A_{f}+B_{f}^{\prime} x_{t}$. First, the SDF given in (9) is rewritten as $M_{t+1}=M_{t+1}^{G} M_{t+1}^{J}$, where

$$
\begin{aligned}
M_{t+1}^{G} & =\beta_{t} \frac{1-\beta_{t+1}}{1-\beta_{t}}\left(\frac{\nu_{t+1}}{\nu_{t}^{1 / \beta_{t}}}\right)^{1-\gamma_{t}} e^{-\gamma_{t} \Delta c_{t+1}^{G}}, \\
M_{t+1}^{J} & =e^{-\gamma_{t}(\ln \xi) N_{t+1}}
\end{aligned}
$$

Then, by the Euler equation, $r_{f, t+1}=-\ln E_{t}\left[M_{t+1}^{G}\right]-\ln E_{t}\left[M_{t+1}^{J}\right]$. The conditional expectation of the Gaussian part is the same as before and that of the Jump part is developed as

$$
-\ln E_{t}\left[e^{-\gamma_{t}(\ln \xi) N_{t+1}}\right]=-l\left(\xi^{-\gamma_{t}}-1\right) .
$$

This term is added to the previous equation for $r_{f, t+1}$ without jumps. To approximate $r_{f, t+1}$ as linear in $x_{t}$, the RHS of (118) is linearized as,

$$
-l\left(\xi^{-\gamma_{t}}-1\right) \approx-l\left(\xi^{-\mu_{\gamma}}-1\right)+l \xi^{-\mu_{\gamma}}(\ln \xi) b_{\gamma}^{\prime} x_{t} .
$$

Then, $r_{f, t+1}=A_{f}+B_{f}^{\prime} x_{t}$, where

$$
\begin{aligned}
A_{f}= & -\ln \left(1-e^{\mu_{\beta}}\right)+\mu_{c}-\frac{1}{2} v_{c \beta}+\left(\mu_{\gamma}-1\right)\left\{\left(s_{x x} b_{\nu}+s_{c x}\right)^{\prime} b_{\beta}-\left(s_{c x}^{\prime} b_{\nu}+s_{c c}\right)\right\} \\
& -l\left(\xi^{-\mu_{\gamma}}-1\right), \\
B_{f}= & b_{c}+\left\{l \xi^{-\mu_{\gamma}} \ln \xi-\left(s_{c x}^{\prime} b_{\nu}+s_{c c}\right)\right\} b_{\gamma}+\left\{\frac{1}{1-e^{\mu_{\beta}}} I_{d \times d}-\Phi_{x}+b_{\gamma}\left(s_{x x} b_{\nu}+s_{c x}\right)^{\prime}\right\} b_{\beta}(1,
\end{aligned}
$$




\section{Appendix B: Condition for the continuation value to be real}

For a state-dependent subjective discount factor, $\beta\left(x_{t}\right)$, the continuation value is approximated as $\nu_{t}=\exp \left\{\mu_{\nu}+b_{\nu}^{\prime} x_{t}\right\}$, where $\mu_{\nu}$ and $b_{\nu}$ are the solution to the simultaneous quadratic equations given in (29) and (30). This appendix presents the condition on which $\mu_{\nu}$ and $b_{\nu}$ are real. Also, it addresses which real root to select.

Recall that (29) and (30) are, respectively,

$$
\begin{aligned}
\mu_{\nu} & =\beta_{0}\left\{\mu_{\nu}+\mu_{c}-\frac{1}{2} v_{c \nu}\left(\mu_{\gamma}-1\right)\right\} \\
b_{\nu} & =\beta_{0}\left(b_{c}+\Phi_{x} b_{\nu}-\frac{1}{2} v_{c \nu} b_{\gamma}\right)+\beta_{1}\left\{\mu_{\nu}+\mu_{c}-\frac{1}{2} v_{c \nu}\left(\mu_{\gamma}-1\right)\right\},
\end{aligned}
$$

where $v_{c \nu}=b_{\nu}^{\prime} s_{x x} b_{\nu}+2 s_{c x}^{\prime} b_{\nu}+s_{c c}$. By (29), $v_{c \nu}$ can be expressed as a linear function of $\mu_{\nu}$. Then, substitute this into (30) and rearrange the terms:

$$
b_{\nu}=c_{0}+c_{1} \mu_{\nu}
$$

where

$$
\begin{aligned}
& c_{0}=\left(I_{d \times d}-\beta_{0} \Phi_{x}\right)^{-1} \beta_{0}\left(b_{c}-\frac{\mu_{c}}{\mu_{\gamma}-1} b_{\gamma}\right), \\
& c_{1}=\left(I_{d \times d}-\beta_{0} \Phi_{x}\right)^{-1}\left(\frac{\beta_{1}}{\beta_{0}}+\frac{1-\beta_{0}}{\mu_{\gamma}-1} b_{\gamma}\right) .
\end{aligned}
$$

Substituting (122) back into (29) yields a quadratic equation with respect to $\mu_{\nu}$ as

$$
\alpha_{2} \mu_{\nu}^{2}+2 \alpha_{1} \mu_{\nu}+\alpha_{0}=0
$$

where

$$
\begin{aligned}
\alpha_{2} & =c_{1}^{\prime} s_{x x} c_{1}, \\
\alpha_{1} & =c_{0}^{\prime} s_{x x} c_{1}+s_{c x}^{\prime} c_{1}+\frac{1-\beta_{0}}{\beta_{0}\left(\mu_{\gamma}-1\right)}, \\
\alpha_{0} & =c_{0}^{\prime} s_{x x} c_{0}+2 s_{c x}^{\prime} c_{0}+s_{c c}-\frac{2 \mu_{c}}{\mu_{\gamma}-1} .
\end{aligned}
$$

Then, the condition for real $\mu_{\nu}$ is that the determinant of (125) is non-negative:

$$
\alpha_{1}^{2}-\alpha_{2} \alpha_{0} \geq 0
$$

By (122), this is also the condition for real $b_{\nu}$. 


\section{Which root to select}

Given that (125) has two real roots, we always select a larger root in order to avoid a negative value of $\mu_{\nu}$ if a smaller root is negative. It is likely that at least one root is positive for typical sets of parameter values, which is explained as follows. (29) can be rewritten as

$$
\mu_{\nu}=\frac{\beta_{0}}{1-\beta_{0}}\left\{\mu_{c}-\frac{1}{2} v_{c \nu}\left(\mu_{\gamma}-1\right)\right\}
$$

where $\beta_{0}=1-e^{\mu_{\beta}}$. By $\mu_{\beta}<0$, it holds that $0<\beta_{0}<1$ and hence that $\beta_{0} /\left(1-\beta_{0}\right)>0$. Furthermore, it is typically the case that the mean term $\left(\mu_{c}=E\left[\Delta c_{t}\right]\right)$ dominates the (scaled) variance term $\left(v_{c \nu}=\operatorname{var}_{t}\left[\ln \nu_{t+1}+\Delta c_{t+1}\right]\right)$ even if $\mu_{\gamma}$ is large.

\section{Appendix C: Accuracy of approximation to the value function}

We examine the accuracy of the approximation to the value function $\nu_{t}^{A P}=\exp \left\{\mu_{\nu}+b_{\nu}^{\prime} x_{t}\right\}$, where an uppercase letter " $A P$ " is added here to clarify the approximation. Approximation error is defined as

$$
e_{t}=\nu_{t}-\nu_{t}^{A P}=E_{t}\left[\left(\nu_{t+1} e^{\Delta c_{t+1}}\right)^{1-\gamma_{t}}\right]^{\beta_{t} /\left(1-\gamma_{t}\right)}-\exp \left\{\mu_{\nu}+b_{\nu}^{\prime} x_{t}\right\}
$$

Since the true form of $\nu_{t}$ is unknown, it is difficult to evaluate the conditional expectation on the RHS of (131) and hence the error $e_{t}$. We instead compute a pseudo approximation error. First, we decompose $e_{t}$ as $e_{t}=e_{1, t}+e_{2, t}$, where

$$
\begin{aligned}
& e_{1, t}=E_{t}\left[\left(\nu_{t+1} e^{\Delta c_{t+1}}\right)^{1-\gamma_{t}}\right]^{\beta_{t} /\left(1-\gamma_{t}\right)}-E_{t}\left[\left(\nu_{t+1}^{A P} e^{\Delta c_{t+1}}\right)^{1-\gamma_{t}}\right]^{\beta_{t} /\left(1-\gamma_{t}\right)}, \\
& e_{2, t}=E_{t}\left[\left(\nu_{t+1}^{A P} e^{\Delta c_{t+1}}\right)^{1-\gamma_{t}}\right]^{\beta_{t} /\left(1-\gamma_{t}\right)}-\exp \left\{\mu_{\nu}+b_{\nu}^{\prime} x_{t}\right\} .
\end{aligned}
$$

Note that taking the $\log$ of $E_{t}\left[\left(\nu_{t+1}^{A P} e^{\Delta c_{t+1}}\right)^{1-\gamma_{t}}\right]^{\beta_{t} /\left(1-\gamma_{t}\right)}$ yields (96). Then, we compute $e_{2, t}$ as a pseudo approximation error. Therefore, unless $e_{1, t}$ and $e_{2, t}$ offset each other, $e_{2, t}$ undervalues the approximation error, and caution is needed to interpret the following results, which are separated in three cases.

\section{C1. Linear risk aversion $\gamma_{t}$ with $E\left[\gamma_{t}\right]=150$}

Panel A of Table A1 presents $e_{2, t} / \nu_{t}^{A P}$ in percentage terms when $\gamma_{t}$ is linear and parameter values are given in Table 4 . The errors are evaluated when the factors are above or below $k(=1,2,3)$ standard deviations from the mean (i.e., zero). By construction of the approximation, $e_{2, t}=0$ 
at $x_{t}=0$. The label "All factors" indicates that all factors change simultaneously, whereas the label "Individual factors" indicates that only a factor in each row changes with the other factors fixed at the mean. Note that the errors in the $x_{\pi, t}$-row are zero as $\nu_{t}$ does not originally depend on $x_{\pi, t}$.

First, by changing all elements of $x_{t}$ proportionally, the pseud errors are at most $-0.12 \%$. Second, by changing only the expected-dividend-growth factor $x_{d, t}$ or the risk-free-rate factor $x_{f, t}$, the pseud errors are negligibly small. Third, since the price-of-risk factor $x_{\lambda, t}$ varies more intensively than the other factors, it is expected to have a larger impact on the accuracy of the approximation. This is indeed the case, but still the pseud errors are at most $0.1 \%$.

\section{C2. Quadratic $\gamma_{t}$ with $E\left[\gamma_{t}\right]=150$}

In Section 4.3, we refer to a quadratic specification of $\gamma_{t}$ to avoid negative values of $\gamma_{t}$. We specify $\gamma_{t}=q_{0}+q_{1} x_{\lambda, t}^{2}$ with $q_{0}, q_{1}>0$. In order to derive $\ln \nu_{t}$ as a linear function of $x_{t}, \gamma_{t}$ needs to be linearized around $x_{\lambda, t}=\mu_{\lambda}$ :

$$
\gamma_{t} \approx q_{0}+q_{1} \mu_{\lambda}^{2}+2 q_{1} \mu_{\lambda}\left(x_{\lambda, t}-\mu_{\lambda}\right)
$$

By matching the intercept and slope terms between (48) and (134), we have $q_{1}=b_{\gamma^{4}} /\left(2 \mu_{\lambda}\right)$ and $q_{0}=\mu_{\gamma}-q_{1} \mu_{\lambda}^{2}$. By this matching, we do not re-calibrate the parameters of the model but simply use those presented in Tables 2 and 4 .

Panel B of Table A1 presents $e_{2, t} / \nu_{t}^{A P}$ in percentage terms for a quadratic $\gamma_{t}$. As expected, the pseud errors are larger than those for a linear $\gamma_{t}$ due to the additional approximation given in (134). Still, they are less than $1 \%$ in absolute value.

\section{C3. Linear $\gamma_{t}$ and jumps in cash flow processes}

To obtain $\ln \nu_{t}^{A P}=\mu_{\nu}+b_{\nu}^{\prime} x_{t}$ in the case of jumps, we need to rely on a further approximation presented in (111). It can be avoided if $\gamma_{t}$ is constant: Remember that $\beta_{t}$ has already been approximated, the accuracy of which is not a serious concern as $\beta_{t}$ does not vary largely. It then follows that the smaller the variation in $\gamma_{t}$, the more accurate is the additional approximation. In Section 5.1, we reduce the volatility of $x_{\lambda, t}$ and hence the volatility of $\gamma_{t}=\mu_{\gamma}+b_{\gamma 4} x_{\lambda, t}$, which is illustrated in Table 6 and Figure 9(a). We can expect therefore that the accuracy is maintained. 
Panel $\mathrm{C}$ of Table A1 presents $e_{2, t} / \nu_{t}^{A P}$ in percentage terms, which are computed at parameter values given implicitly in Table 6 . As expected, the additional approximation is not a serious concern. The pseud error is at most $-0.2 \%$ when all components of the state vector are above three standard deviations from the mean. In changing the value of each factor, there is no case in which the pseud error exceeds $0.1 \%$ in absolute value.

\section{Appendix D: Term structure formulas}

\section{Risk-neutral drift}

To simplify the notation of recursive equations for the prices of zero-coupon bonds and equities, we bundle model parameters into those in the risk-neutral probability measure. Specifically, we first describe the risk-neutral dynamics as

$$
\begin{aligned}
\Delta d_{t+1} & =\mu_{d}^{Q}+b_{d}^{Q \prime} x_{t}+\sigma_{d}^{\prime} z_{t+1}^{Q}, \\
\Delta \pi_{t+1} & =\mu_{\pi}^{Q}+b_{\pi}^{Q \prime} x_{t}+\sigma_{\pi}^{\prime} z_{t+1}^{Q}, \\
x_{t+1} & =\mu_{x}^{Q}+\Phi_{x}^{Q \prime} x_{t}+\sigma_{x}^{\prime} z_{t+1}^{Q},
\end{aligned}
$$

where $z_{t+1}^{Q}$ is an i.i.d. normal random vector in the risk-neutral probability measure. The risk-neutral drift of $\Delta d_{t+1}$ satisfies for any $x_{t}$

$$
\mu_{d}^{Q}+b_{d}^{Q \prime} x_{t}=E_{t}\left[\Delta d_{t+1}\right]+\operatorname{cov}_{t}\left[m_{t+1}, \Delta d_{t+1}\right] .
$$

The conditional covariance on the RHS of (138) is

$$
\operatorname{cov}_{t}\left[\Delta d_{t+1}, m_{t, t+1}\right]=-\sigma_{d}^{\prime} \lambda_{t}=s_{d x}^{\prime}\left(b_{\beta}+b_{\nu}\right)-\left(s_{d x}^{\prime} b_{\nu}+s_{c d}\right) \mu_{\gamma}-\left(s_{d x}^{\prime} b_{\nu}+s_{c d}\right) b_{\gamma}^{\prime} x_{t} .
$$

Then, $\mu_{d}^{Q}$ and $b_{d}^{Q}$ on the LHS of (138) are identified as

$$
\mu_{d}^{Q}=\mu_{d}+s_{d x}^{\prime}\left(b_{\beta}+b_{\nu}\right)-\mu_{\gamma}\left(s_{d x}^{\prime} b_{\nu}+s_{c d}\right), \quad b_{d}^{Q}=b_{d}-b_{\gamma}\left(s_{d x}^{\prime} b_{\nu}+s_{c d}\right) .
$$

Likewise,

$$
\begin{array}{cl}
\mu_{\pi}^{Q}=\mu_{\pi}+s_{\pi x}^{\prime}\left(b_{\beta}+b_{\nu}\right)-\mu_{\gamma}\left(s_{\pi x}^{\prime} b_{\nu}+s_{c \pi}\right), & b_{\pi}^{Q}=b_{\pi}-b_{\gamma}\left(s_{\pi x}^{\prime} b_{\nu}+s_{c \pi}\right), \\
\mu_{x}^{Q}=s_{x x}\left(b_{\beta}+b_{\nu}\right)-\mu_{\gamma}\left(s_{x x} b_{\nu}+s_{c x}\right), & \Phi_{x}^{Q}=\Phi_{x}-b_{\gamma}\left(s_{x x} b_{\nu}+s_{c x}\right)^{\prime} .
\end{array}
$$

By the calibration, the slope terms of the factor risk premiums are matched exactly between the LW and proposed models. This is equivalent to matching the slope terms of the risk-neutral drift (i.e., $b_{d}^{Q}, b_{\pi}^{Q}$, and $\Phi_{x}^{Q}$ ) between the two models. 


\section{Real zero-coupon bonds}

The price of a real zero-coupon bond maturing in $n$ periods, $P_{t, n}^{R}$, satisfies the following Euler equation:

$$
P_{t, n}^{R}=E_{t}\left[M_{t+1} P_{t+1, n-1}^{R}\right]=e^{-r_{f, t+1}} E_{t}^{Q}\left[P_{t+1, n-1}^{R}\right],
$$

where the second equality is due to the change from the physical to risk-neutral probability measures and $E_{t}^{Q}[\cdot]$ stands for the conditional expectation under the risk-neutral probability measure. The initial condition is $P_{t, 0}^{R}=1$. By substituting $P_{t, n}^{R}=\exp \left\{A_{n}^{R}+B_{n}^{R \prime} x_{t}\right\}$ into the RHS of (143), developing the conditional expectation under the risk-neutral probability measure, and matching the intercept and slope terms on both sides, we obtain the following recursive equations for $A_{n}^{R}$ and $B_{n}^{R}$ :

$$
\begin{aligned}
& A_{n}^{R}=A_{n-1}^{R}-A_{f}+\mu_{x}^{Q \prime} B_{n-1}^{R}+\frac{1}{2} B_{n-1}^{R \prime} s_{x x} B_{n-1}^{R}, \\
& B_{n}^{R}=\Phi_{x}^{Q} B_{n-1}^{R}-B_{f},
\end{aligned}
$$

with the initial condition $A_{0}^{R}=0$ and $B_{0}^{R}=0$.

It is noted that $\Phi_{x}^{Q}$ in (145) is the same between the LW and proposed models as documented above. Also, $B_{f}$, the loading on the state vector for the real risk-free rate $r_{f, t+1}$, is the same between the two models as (50) holds by the calibration procedure. Consequently, $B_{n}^{R}$ is the same between the two models for any $n$.

\section{Nominal zero-coupon bonds}

Rewrite the Euler equation (38) for the real price of a nominal zero-coupon bond as

$$
P_{t, n}^{N} \Pi_{t}=E_{t}\left[M_{t+1}\left(P_{t+1, n-1}^{N} \Pi_{t+1}\right) \frac{\Pi_{t}}{\Pi_{t+1}}\right]=e^{-r_{f, t+1}} E_{t}^{Q}\left[\left(P_{t+1, n-1}^{N} \Pi_{t+1}\right) \frac{\Pi_{t}}{\Pi_{t+1}}\right],
$$

with the initial condition $P_{t, 0}^{N} \Pi_{t}=1$. By substituting $P_{t, n}^{N} \Pi_{t}=\exp \left\{A_{n}^{N}+B_{n}^{N \prime} x_{t}\right\}$ into (146), developing the conditional expectation under the risk-neutral probability measure, and matching the intercept and slope terms on both sides, we obtain the following recursive equations for $A_{n}^{N}$ and $B_{n}^{N}$ :

$$
\begin{aligned}
& A_{n}^{N}=A_{n-1}^{N}-A_{f}-\mu_{\pi}^{Q}+\frac{1}{2} s_{\pi \pi}+\left(\mu_{x}^{Q}-s_{\pi x}\right)^{\prime} B_{n-1}^{N}+\frac{1}{2} B_{n-1}^{N \prime} s_{x x} B_{n-1}^{N}, \\
& B_{n}^{N}=\Phi_{x}^{Q} B_{n-1}^{N}-B_{f}-b_{\pi}^{Q},
\end{aligned}
$$

with the initial condition $A_{0}^{N}=0$ and $B_{0}^{N}=0$. Notice that $B_{n}^{N}$ is the same between the LW and proposed models for any $n$ because $\Phi_{x}^{Q}, B_{f}$, and $b_{\pi}^{Q}$ are the same between the two models by the calibration. 


\section{Zero-coupon equities}

Rewrite the Euler equation (40) for the price of a dividend strip as

$$
\frac{P_{t, n}^{D}}{D_{t}}=E_{t}\left[M_{t+1} \frac{P_{t+1, n-1}^{D}}{D_{t+1}} \frac{D_{t+1}}{D_{t}}\right]=e^{-r_{f, t+1}} E_{t}^{Q}\left[\frac{P_{t+1, n-1}^{D}}{D_{t+1}} e^{\Delta d_{t+1}}\right],
$$

with the initial condition $P_{t, 0}^{D} / D_{t}=1$. By substituting $P_{t, n}^{D} / D_{t}=\exp \left\{A_{n}^{D}+B_{n}^{D \prime} x_{t}\right\}$ into (149), developing the conditional expectation under the risk-neutral probability measure, and matching the intercept and slope terms on both sides, we obtain the following recursive equations for $A_{n}^{D}$ and $B_{n}^{D}$ :

$$
\begin{aligned}
& A_{n}^{D}=A_{n-1}^{D}-A_{f}+\mu_{d}^{Q}+\frac{1}{2} s_{d d}+\left(\mu_{x}^{Q}+s_{d x}\right)^{\prime} B_{n-1}^{D}+\frac{1}{2} B_{n-1}^{D \prime} s_{x x} B_{n-1}^{D}, \\
& B_{n}^{D}=\Phi_{x}^{Q} B_{n-1}^{D}-B_{f}+b_{d}^{Q},
\end{aligned}
$$

with $A_{0}^{D}=0$ and $B_{0}^{D}=0$. For the same reason as above, $B_{n}^{D}$ is the same between the LW and proposed models for any $n$.

\section{Zero-coupon equities with jumps in consumption and dividend growth}

The SDE $M_{t+1}$ is decomposed into Gaussian and jump components, which are mutually independent, as

$$
M_{t+1}=M_{t+1}^{G} M_{t+1}^{J}=e^{-r_{f, t+1}} \frac{M_{t+1}^{G}}{E_{t}\left[M_{t+1}^{G}\right]} \frac{M_{t+1}^{J}}{E_{t}\left[M_{t+1}^{J}\right]} .
$$

Also, $\Delta d_{t+1}=\Delta d_{t+1}^{G}+\Delta d_{t+1}^{J}$. Meanwhile, $P_{t+1, n-1}^{D} / D_{t+1}$ does not depend on a jump component as it is a function of $x_{t+1}$ that has no jump component. Then, the recursive equation (149) is developed as

$$
\frac{P_{t, n}^{D}}{D_{t}}=e^{-r_{f, t+1}} E_{t}\left[\frac{M_{t+1}^{G}}{E_{t}\left[M_{t+1}^{G}\right]} \frac{P_{t+1, n-1}^{D}}{D_{t+1}} e^{\Delta d_{t+1}^{G}}\right] E_{t}\left[\frac{M_{t+1}^{J}}{E_{t}\left[M_{t+1}^{J}\right]} e^{\Delta d_{t+1}^{J}}\right] .
$$

The first conditional expectation on the RHS of (152) is the same as that developed without jumps. The second conditional expectation is developed by substituting $M_{t+1}^{J}=e^{-\gamma_{t}(\ln \xi) N_{t+1}}$ and $\Delta d_{t+1}^{J}=k(\ln \xi) N_{t+1}$ as

$$
E_{t}\left[\exp \left\{\left(k-\gamma_{t}\right)(\ln \xi) N_{t+1}-l\left(\xi^{-\gamma_{t}}-1\right)\right\}\right]=\exp \left\{l\left(\xi^{k}-1\right) \xi^{-\gamma_{t}}\right\}
$$

To derive $\ln \left(P_{t, n}^{D} / D_{t}\right)$ as a linear function of $x_{t}, \xi^{-\gamma_{t}}$ is linearized as

$$
\xi^{-\gamma_{t}} \approx \xi^{-\mu_{\gamma}}\left\{1-(\ln \xi) b_{\gamma}^{\prime} x_{t}\right\}
$$


Then, $\ln \left(P_{t, n}^{D} / D_{t}\right)=A_{n}^{D}+B_{n}^{D \prime} x_{t}$, where $A_{n}^{D}$ and $B_{n}^{D}$ are determined recursively as

$$
\begin{aligned}
& A_{n}^{D}=A_{n-1}^{D}-A_{f}+\mu_{d}^{Q}+\frac{1}{2} s_{d d}+\left(\mu_{x}^{Q}+s_{d x}\right)^{\prime} B_{n-1}^{D}+\frac{1}{2} B_{n-1}^{D \prime} s_{x x} B_{n-1}^{D}+l\left(\xi^{k}-1\right) \xi^{-\mu_{\gamma}}(155) \\
& B_{n}^{D}=\Phi_{x}^{Q} B_{n-1}^{D}-B_{f}+b_{d}^{Q}-l\left(\xi^{k}-1\right) \xi^{-\mu_{\gamma}}(\ln \xi) b_{\gamma},
\end{aligned}
$$

with $A_{0}^{D}=0$ and $B_{0}^{D}=0$. 


\section{References}

Abel, A. B., 1999, Risk Premia and Term Premia in General Equilibrium, Journal of Monetary Economics 43, 3-33.

Bansal, R., A. R. Gallant, and G. Tauchen, 2007, Rational Pessimism, Rational Exuberance, and Asset Pricing Models, Review of Economic Studies 74, 1005-1033.

Bansal, R., D. Kiku, and A. Yaron, 2012, An Empirical Evaluation of the Long-Run Risks Model for Asset Prices, Critical Financial Review 1, 183-221.

Bansal, R., and I. Shaliastovich, 2013, A Long-Run Risks Explanation of Predictability Puzzles in Bond and Currency Markets, Review of Financial Studies 26, 1-33.

Bansal, R., and A. Yaron, 2004, Risks for the Long Run: A Potential Resolution of Asset Pricing Puzzles, Journal of Finance 59, 1481-1509.

Barro, R. J., 2006, Rare Disasters and Asset Markets in the Twentieth Century, Quarterly Journal of Economics 212, 823-866.

Barro, R. J., 2009, Rare Disasters, Asset Prices, and Welfare Costs, American Economic Review 99, 243-264.

Beeler, J., and J. Y. Campbell, 2012, The Long-Run Risks Model and Aggregate Asset Prices: An Empirical Assessment, Critical Financial Review 1, 141-182.

Belo, F., P. Collin-Dufresne, and R. S. Goldstein, 2015, Dividend Dynamics and the Term Structure of Dividend Strips, Journal of Finance 70, 1115-1160.

Berrada, T., J. Detemple, and M. Rindisbacher, 2013, Asset Pricing with Regime-Dependent Preferences and Learning, Working Paper.

Binsbergen, van, J. H., M. Brandt, and R. S. J. Koijen, 2012, On the Timing and Pricing of Dividends, American Economic Review 102, 1596-1618.

Binsbergen, van, J. H., J. Fernandez-Villaverde, R. S. J. Koijen, and J. Rubio-Ramirez, 2012, The Term Structure of Interest Rates in a DSGE Model with Recursive Preferences, Journal of Monetary Economics 59, 634-648. 
Binsbergen, van, J. H., W. Hueskes, R. Koijen, and E. Vrugt, 2013, Equity Yields, Journal of Financial Economics 110, 503-519.

Binsbergen, van, J. H., and R. S. J. Koijen, 2017, The Term Structure of Returns: Facts and Theory, Journal of Financial Economics 124, 1-21.

Campbell, J. Y., 2003, Consumption-based Asset Pricing, Ch.13 in G. Constantinides, M. Harris, and R. Stulz eds., Handbook of the Economics of Finance IB, North-Holland, Amsterdam, 803887.

Campbell, J. Y., and J. H. Cochrane, 1999, By Force of Habit: A Consumption-Based Explanation of Aggregate Stock Market Behavior, Journal of Political Economy 107, 205-251.

Chabi-Yo, F., R. Garcia, and E. Renault, 2008, State Dependence Can Explain the Risk Aversion Puzzle, Review of Financial Studies 21, 973-1011.

Croce, M. M., M. Lettau, and S. C. Ludvigson, 2015, Investor Information, Long-Run Risk, and the Term Structure of Equity, Review of Financial Studies 28, 706-742.

Curatola, G., 2015, Loss Aversion, Habit Formation and the Term Structures of Equity and Interest Rates, Journal of Economic Dynamics and Control 53, 103-122.

Dew-Becker, I., 2014, Bond Pricing with a Time-Varying Price of Risk in an Estimated MediumScale Bayesian DSGE Model, Journal of Money, Credit and Banking 46, 837-888.

Doh, T., and S. Wu, 2016, The Equilibrium Term Structure of Equity and Interest Rates, Working Paper.

Epstein, L. G., and S. E. Zin, 1989, Substitution, Risk Aversion, and the Temporal Behavior of Consumption and Asset Returns: A Theoretical Framework, Econometrica 57, 937-969.

Epstein, L. G., and S. E. Zin, 1991, Substitution, Risk Aversion, and the Temporal Behavior of Consumption and Asset Returns: An Empirical Analysis, Journal of Political Economy 99, 263-286.

Favilukis, J., and X. Lin, 2016, Wage Rigidity: A Quantitative Solution to Several Asset Pricing Puzzles, Review of Financial Studies 29, 148-192.

Frederick, S., G. Loewenstein, and T. O'Donoghue, 2002, Time Discounting and Time Preference: A Critical Review, Journal of Economic Literature 40, 351-401. 
Gabaix, X., 2012, Variable Rare Disasters: An Exactly Solved Framework for Ten Puzzles in Macro-Finance, Quarterly Journal of Economics 127, 645-700.

Gordon, S., and P. St-Amour, 2000, A Preference Regime Model of Bull and Bear Markets, American Economic Review 90, 1019-1033.

Gordon, S., and P. St-Amour, 2004, Asset Returns and State-Dependent Risk Preferences, Journal of Business and Economic Statistics 22, 241-252.

Grossman, S. J., and R. J. Shiller, 1981, The Determinants of the Variability of Stock Market Prices, American Economic Review 71, 222-227.

Halevy, Y., 2015, Time Consistency: Stationarity and Time Invariance, Econometrica 83, 335352.

Hansen, L. P., J. C. Heaton, and N. Li, 2008, Consumption Strikes Back? Measuring Long-Run Risk, Journal of Political Economy 116, 260-302.

Hansen, L. P., J. C. Heaton, J. Lee, and N. Roussanov, 2007, Intertemporal Substitution and Risk Aversion, Handbook of Econometrics Ch.61, 3968-4056.

Harris, C., and D. Laibson, 2001, Dynamic Choices of Hyperbolic Consumers, Econometrica 69, 935-957.

Hasler, M., and R. Marfè, 2016, Disaster Recovery and the Term Structure of Dividend Strips, Journal of Financial Economics 122, 116-134.

Lettau, M., and J. A. Wachter, 2011, The Term Structures of Equity and Interest Rates, Journal of Financial Economics 101, 90-113.

Lopez, P., D. Lopez-Salido, and F. Vazquez-Grande, 2015, Nominal Rigidities and the Term Structures of Equity and Bond Returns, Working Paper.

Luttmer, E. G. J., and T. Mariotti, 2003, Subjective Discounting in an Exchange Economy, Journal of Political Economy 111, 959-989.

Marfè, R., 2017, Insurance and the Equilibrium Term Structure of Equity, Journal of Finance $72,2073-2130$. 
Mehra, R., and E. C. Prescott, 1985, The equity premium: A puzzle, Journal of Monetary Economics 15, 145-161.

Melino, A., and A. X. Yang, 2003, State-Dependent Preferences Can Explain the Equity Premium Puzzle, Review of Economic Dynamics 6, 806-830.

Piazzesi, M., and M. Schneider, 2006, Equilibrium Yield Curves, NBER Macroeconomics Annual 21.

Rietz, T. A., 1988, The Equity Risk Premium: A Solution, Journal of Monetary Economics 22, 117-131.

Rudebusch, G. D., and E. T. Swanson, 2012, The Bond Premium in a DSGE Model with Long-Run Real and Nominal Risks, American Economic Journal: Macroeconomics 4, 105-143.

Schulz, F., 2016, On the Timing and Pricing of Dividends: Comment, American Economic Review 106, 3185-3223.

Thaler, R. H., 1981, Some Empirical Evidence on Dynamic Inconsistency, Economics Letters 8, 201-207.

Wachter, J. A., 2006, A Consumption-based Model of the Term Structure of Interest Rates, Journal of Financial Economics 79, 365-399.

Wachter, J. A., 2013, Can Time-Varying Risk of Rare Disasters Explain Aggregate Stock Market Volatility?, Journal of Finance 68, 987-1035.

Weber, M., 2018, Cash Flow Duration and the Term Structure of Equity Returns, Journal of Financial Economics 128, 486-503.

Weil, P., 1989, The Equity Premium Puzzle and the Risk-free Rate Puzzle, Journal of Monetary Economics 24, 401-421. 


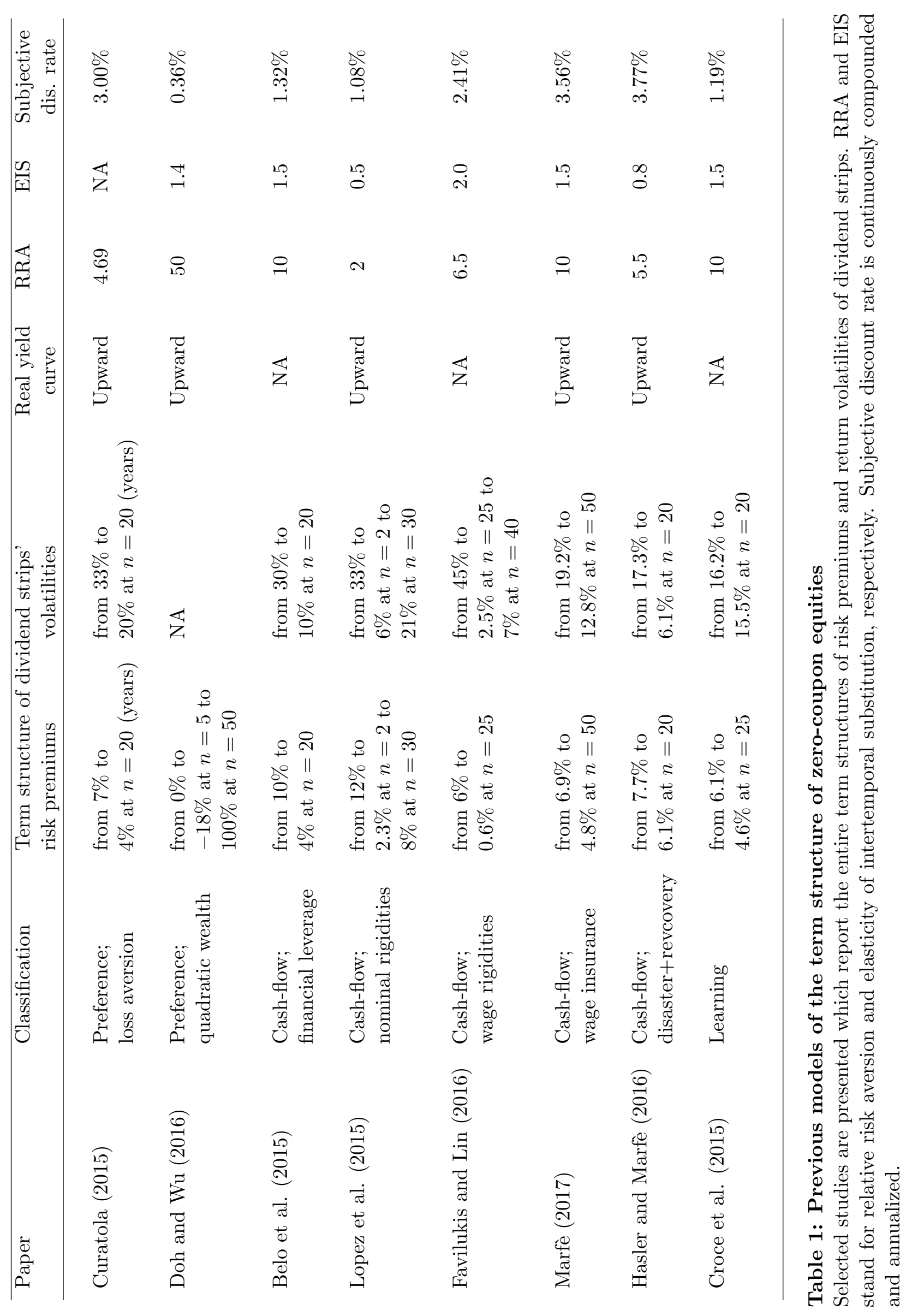




\begin{tabular}{|c|c|c|c|c|c|c|}
\hline & $\Delta d$ & $\Delta \pi$ & $x_{d}$ & $x_{\pi}$ & $x_{f}$ & $x_{\lambda}$ \\
\hline \multicolumn{7}{|c|}{ Unconditional means } \\
\hline$\mu$. & $1.29 \%$ & $3.68 \%$ & - & - & $0.96 \%$ & 17.0 \\
\hline \multicolumn{7}{|c|}{ Standard deviations of innovation terms } \\
\hline$\sqrt{s_{. .}}$ & $10.0 \%$ & $1.18 \%$ & $0.32 \%$ & $0.35 \%$ & $0.19 \%$ & 4.00 \\
\hline \multicolumn{7}{|c|}{ Autocorrelations } \\
\hline $\operatorname{diag}\left(\phi_{x .}\right)$ & - & - & 0.90 & 0.78 & 0.92 & 0.85 \\
\hline \multicolumn{7}{|c|}{ Correlations between innovation terms } \\
\hline$\Delta d$ & 1.00 & -0.30 & -0.83 & -0.30 & -0.30 & 0.00 \\
\hline$\Delta \pi$ & & 1.00 & 0.00 & 1.00 & 0.00 & 0.00 \\
\hline$x_{d}$ & & & 1.00 & 0.00 & 0.00 & 0.35 \\
\hline$x_{\pi}$ & & & & 1.00 & 0.00 & 0.00 \\
\hline$x_{f}$ & & & & & 1.00 & 0.00 \\
\hline \multicolumn{7}{|c|}{ Unconditional factor risk premiums } \\
\hline & $17.00 \%$ & $-0.60 \%$ & $-0.45 \%$ & $-0.18 \%$ & $-0.10 \%$ & $0.00 \%$ \\
\hline
\end{tabular}

\section{Table 2: Parameter values of the LW model}

These values are collected from tables 1-3 in LW (2011). Unconditional means, standard deviations, and autocorrelations are annualized, except for the unconditional mean of $x_{\lambda, t}$ and the standard deviation of innovation in $x_{\lambda, t}$ expressed in raw numbers. The last row presents annualized, unconditional factor risk premiums, except for $x_{\lambda}$ expressed in row numbers. 


\begin{tabular}{|c|c|c|c|c|c|}
\hline \multirow[t]{2}{*}{ Solution } & \multirow{2}{*}{$\begin{array}{c}\text { Consumption } \\
\sqrt{s_{c c}} \\
(\%, \text { year }) \\
\end{array}$} & \multicolumn{2}{|c|}{ Risk aversion } & \multicolumn{2}{|c|}{ Subjective discount factor } \\
\hline & & $E\left[\gamma_{t}\right]$ & $S D\left[\gamma_{t}\right]$ & $E\left[\beta_{t}\right]$ & $\begin{array}{l}S D\left[\beta_{t}\right] \\
\left(\times 10^{2}\right)\end{array}$ \\
\hline (a) & 8.84 & 30 & 25 & 0.969 & 1.097 \\
\hline (b) & 5.89 & 60 & 51 & 0.981 & 0.532 \\
\hline (c) & 4.77 & 90 & 77 & 0.985 & 0.392 \\
\hline (d) & 4.22 & 120 & 102 & 0.986 & 0.331 \\
\hline (e) & 3.91 & 150 & 128 & 0.987 & 0.298 \\
\hline
\end{tabular}

Table 3: Moments for consumption and preferences at selected solutions

Table 3 presents the annualized volatility of innovation in consumption growth $\left(\sqrt{s_{c c}}\right)$, and the unconditional mean $(E[\cdot])$ and standard deviation $(S D[\cdot])$ of state-dependent preferences at selected solutions to the set of constraint equations given in Section 3.2. The solutions are in ascending order of $E\left[\gamma_{t}\right]$. 
Panel A: Consumption volatility and correlations

\begin{tabular}{cccccc}
$\sqrt{s_{c c}}$ & $\rho_{c d}$ & $\rho_{c x 1}$ & $\rho_{c x 2}$ & $\rho_{c x 3}$ & $\rho_{c x 4}$ \\
\hline $3.91 \%$ & 0.877 & -0.755 & -0.086 & -0.046 & 0.341
\end{tabular}

Panel B: State-dependent preferences and continuation value

\begin{tabular}{lrrrrr} 
& constant & $x_{d}$ & $x_{\pi}$ & \multicolumn{1}{c}{$x_{f}$} & \multicolumn{1}{c}{$x_{\lambda}$} \\
\cline { 2 - 6 }$\gamma_{t}$ & 150 & 0.00 & 0.00 & 0.000 & 8.9357 \\
$\ln \left(1-\beta_{t}\right)$ & -4.392 & -8.66 & 0.00 & 30.170 & 0.0136 \\
$\ln \nu_{t}$ & 0.071 & 8.86 & 0.00 & -0.817 & -0.0029
\end{tabular}

Panel C: Unconditional factor risk premiums

\begin{tabular}{cccccc}
$\Delta d$ & $\Delta \pi$ & $x_{d}$ & $x_{\pi}$ & $x_{f}$ & $x_{\lambda}$ \\
\hline $17.00 \%$ & $-0.60 \%$ & $-0.46 \%$ & $-0.18 \%$ & $-0.11 \%$ & -0.172
\end{tabular}

Table 4: Implied parameters of consumption dynamics and preferences

Table 4 presents calibrated parameters at Solution (e) of Table 3. Panel A presents the annualized volatility of innovation in consumption growth $\left(\sqrt{s_{c c}}\right)$ and the correlations between innovations in consumption growth and the rest of the variables. The correlation with realized inflation growth is not shown because it is the same as the correlation with expected inflation growth $\left(\rho_{c x 2}\right)$. Panel B presents the parameters in the risk aversion $\gamma_{t}$, the log subjective discount rate $\ln \left(1-\beta_{t}\right)$, and the $\log$ continuation value $\ln \nu_{t}$. These functions are all linear in $x_{t}^{\prime}=\left(x_{d, t}, x_{\pi, t}, x_{f, t}, x_{\lambda, t}\right)$. Panel $\mathrm{C}$ presents annualized, unconditional factor risk premiums, except for $x_{\lambda}$ expressed in row numbers. 
Panel A: Consumption volatility and correlations

\begin{tabular}{cccccc}
$\sqrt{s_{c c}}$ & $\rho_{c d}$ & $\rho_{c x 1}$ & $\rho_{c x 2}$ & $\rho_{c x 3}$ & $\rho_{c x 4}$ \\
\hline $4.96 \%$ & 0.898 & -0.792 & -0.069 & 0.085 & 0.281
\end{tabular}

Panel B: State-dependent preferences and continuation value

\begin{tabular}{lrrcrr} 
& constant & $x_{d}$ & $x_{\pi}$ & \multicolumn{1}{c}{$x_{f}$} & \multicolumn{1}{c}{$x_{\lambda}$} \\
\cline { 2 - 6 }$\gamma_{t}$ & 150 & 0.00 & 0.00 & 0.000 & 8.7817 \\
$\ln \left(1-\beta_{t}\right)$ & -6.337 & -12.00 & 0.00 & 44.639 & 0.0025 \\
$\ln \nu_{t}$ & 0.458 & 12.36 & 0.00 & -1.619 & -0.0035
\end{tabular}

Panel C: Unconditional factor risk premiums

\begin{tabular}{cccccc}
$\Delta d$ & $\Delta \pi$ & $x_{d}$ & $x_{\pi}$ & $x_{f}$ & $x_{\lambda}$ \\
\hline $17.00 \%$ & $-0.60 \%$ & $-0.45 \%$ & $-0.18 \%$ & $0.02 \%$ & 0.015
\end{tabular}

Table 5: Implied parameters for $\rho_{d x 3}=0.1$

$\rho_{d x 3}$ stands for the correlation between innovations in dividend growth and risk-free-rate factor. It is first changed from -0.3 to 0.1 in the LW model, and then the parameters of the proposed model are re-calibrated in the same procedure as explained in Section 3.2. The same legend as in Table 4 follows. 


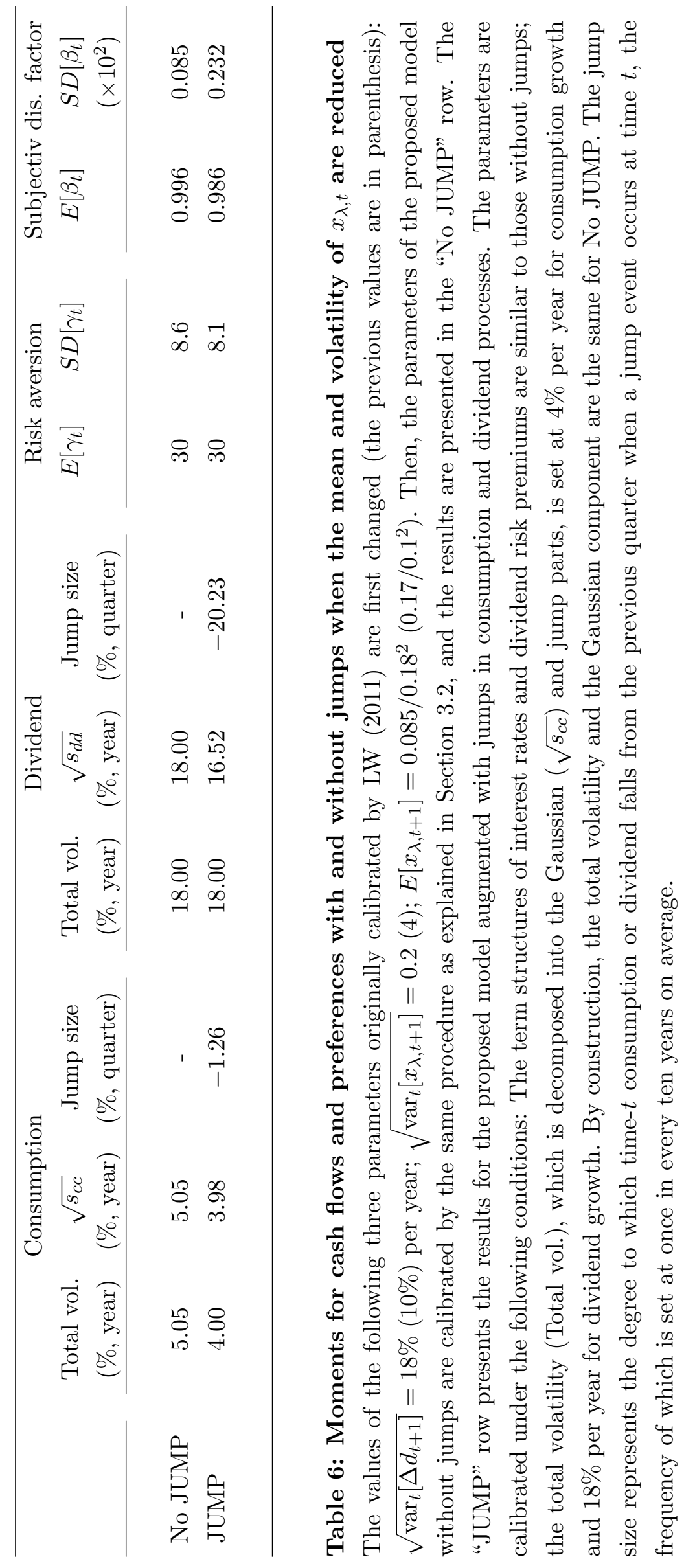




\begin{tabular}{|c|c|c|c|c|c|c|}
\hline & -3 S.D. & -2 S.D. & -1 S.D. & +1S.D. & $+2 \mathrm{~S} . \mathrm{D}$ & +3 S.D. \\
\hline \multicolumn{7}{|c|}{ Panel A: Linear $\gamma_{t}$} \\
\hline All factors & -0.060 & -0.030 & -0.008 & -0.011 & -0.048 & -0.124 \\
\hline \multicolumn{7}{|c|}{ Individual factors } \\
\hline$x_{d}$ & 0.046 & 0.020 & 0.005 & 0.004 & 0.017 & 0.038 \\
\hline$x_{\pi}$ & 0.000 & 0.000 & 0.000 & 0.000 & 0.000 & 0.000 \\
\hline$x_{f}$ & -0.002 & -0.001 & 0.000 & 0.000 & -0.001 & -0.002 \\
\hline$x_{\lambda}$ & 0.056 & 0.028 & 0.008 & 0.009 & 0.042 & 0.105 \\
\hline \multicolumn{7}{|c|}{ Panel B: Quadratic $\gamma_{t}$} \\
\hline All factors & -0.812 & -0.364 & -0.092 & -0.094 & -0.378 & -0.858 \\
\hline \multicolumn{7}{|c|}{ Individual factors } \\
\hline$x_{d}$ & 0.046 & 0.020 & 0.005 & 0.004 & 0.017 & 0.038 \\
\hline$x_{\pi}$ & 0.000 & 0.000 & 0.000 & 0.000 & 0.000 & 0.000 \\
\hline$x_{f}$ & -0.002 & -0.001 & 0.000 & 0.000 & -0.001 & -0.002 \\
\hline$x_{\lambda}$ & -0.695 & -0.307 & -0.076 & -0.074 & -0.289 & -0.635 \\
\hline \multicolumn{7}{|c|}{ Panel C: Linear $\gamma_{t}$ with jumps in consumption and dividend growth } \\
\hline All factors & -0.099 & -0.047 & -0.013 & -0.015 & -0.064 & -0.155 \\
\hline \multicolumn{7}{|c|}{ Individual factors } \\
\hline$x_{d}$ & 0.047 & 0.020 & 0.005 & 0.005 & 0.018 & 0.039 \\
\hline$x_{\pi}$ & 0.000 & 0.000 & 0.000 & 0.000 & 0.000 & 0.000 \\
\hline$x_{f}$ & -0.004 & -0.002 & 0.000 & 0.000 & -0.001 & -0.003 \\
\hline$x_{\lambda}$ & 0.000 & 0.000 & 0.000 & 0.000 & 0.000 & 0.001 \\
\hline
\end{tabular}

\section{Table A1: Approximation errors of $\nu_{t}^{A P}$}

The value function is approximated as $\nu_{t}^{A P}=\exp \left\{\mu_{\nu}+b_{\nu}^{\prime} x_{t}\right\}$. The table reports $e_{2, t} / \nu_{t}^{A P}$ in percentage terms, where $e_{2, t}$ stands for a pseudo approximation error defined as $e_{2, t}=$ $E_{t}\left[\left(\nu_{t+1}^{A P} e^{\Delta c_{t+1}}\right)^{1-\gamma_{t}}\right]^{\beta /\left(1-\gamma_{t}\right)}-\nu_{t}^{A P}$. These errors are evaluated when the values of the factors are above or below $k(=1,2,3)$ standard deviations (S.D.) from the mean (i.e., zero). In each panel, the label "All factors" indicates that all factors change proportionally, whereas the label "Individual factors" indicates that only a factor in each row changes with the other factors fixed at the mean. By construction of the approximation, $e_{2, t}=0$ at $x_{t}=0$. Panels A and B are for a linear risk-aversion and a quadratic risk-aversion, respectively, evaluated at parameter values given in Tables 2 and 4. Panel $\mathrm{C}$ is for a linear risk-aversion with jumps in consumption and dividend processes, evaluated at parameter values given implicitly in Table 6 . 
(a) Risk aversion $\gamma_{t}$

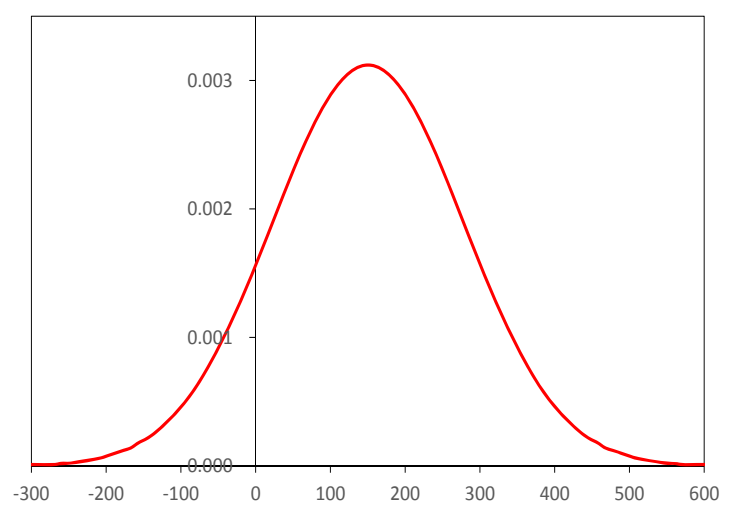

(b) Subjective discount factor $\beta_{t}$

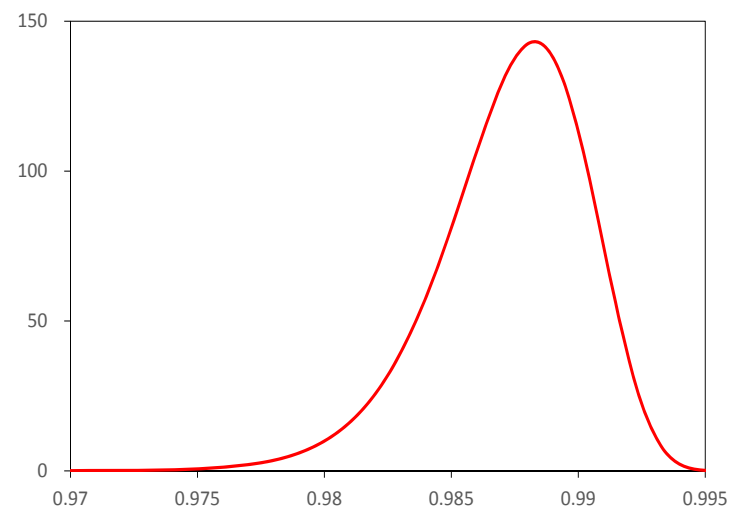

Figure 1: Unconditional distributions of state-dependent preferences

The distributions are drawn at Solution (e) of Table 3. The risk-aversion coefficient is specified as $\gamma_{t}=\mu_{\gamma}+b_{\gamma}^{\prime} x_{t}$ and the subjective discount factor as $\beta_{t}=1-\exp \left\{\mu_{\beta}+b_{\beta}^{\prime} x_{t}\right\}$, where $x_{t}$ is a Gaussian state vector. 
(a) Loading on $x_{d, t}$

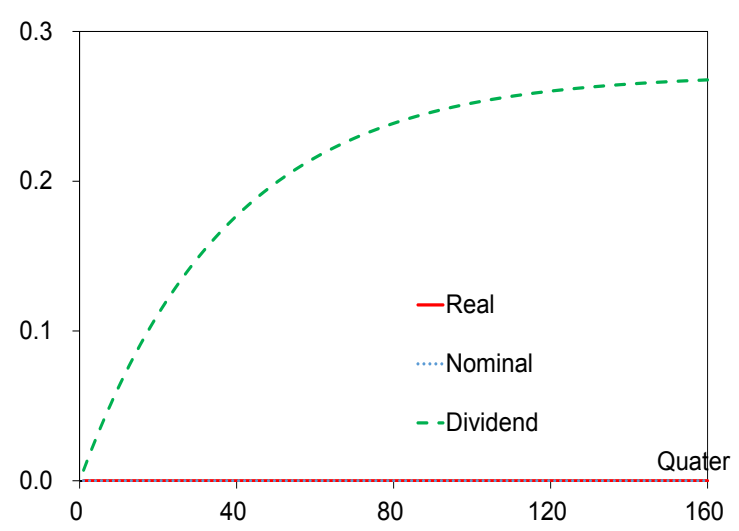

(c) Loading on $x_{f, t}$

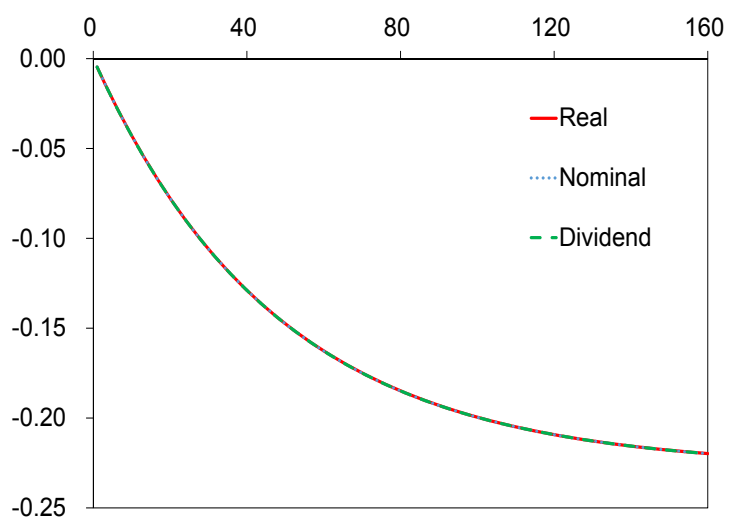

(b) Loading on $x_{\pi, t}$

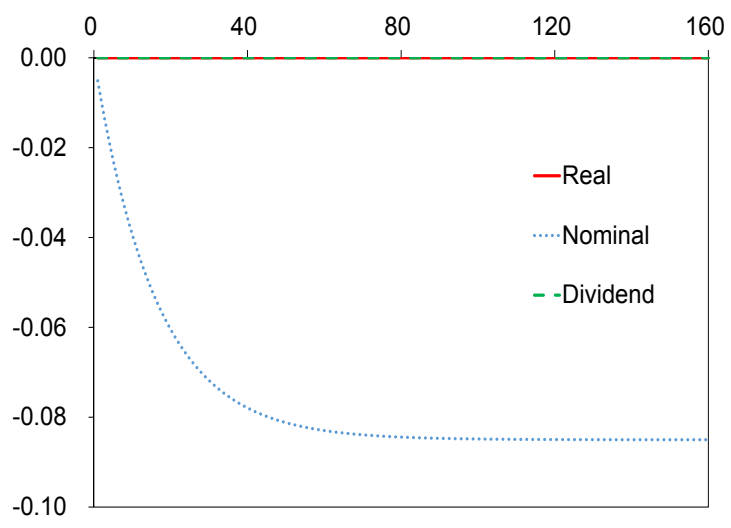

(d) Loading on $x_{\lambda, t}$

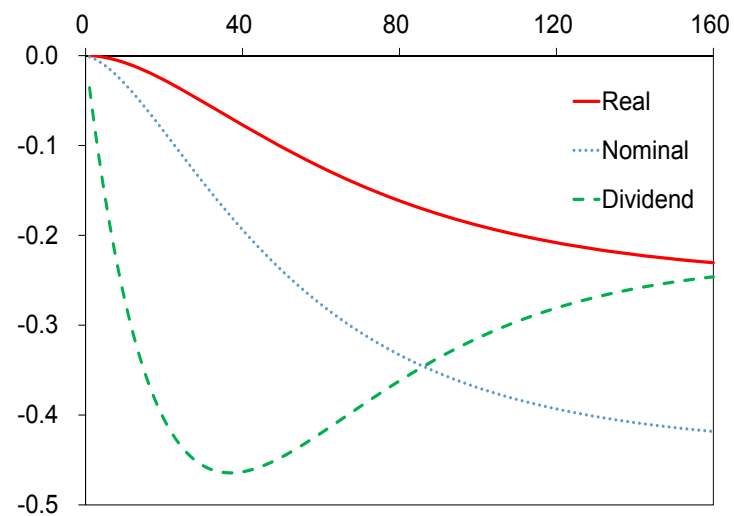

Figure 2: Log price loadings on state variables $B_{n}^{i}(i=\{R, N, D\})$

The log prices for real bonds (R), nominal bonds (N), and dividend strips (D) are given by $\ln P_{t, n}^{i}=A_{n}^{i}+B_{n}^{i \prime} x_{t}(i=\{R, N, D\})$. Panel (a) plots against $n$ (quarters) the loadings on the expected dividend-growth factor $x_{d, t}$, divided by the unconditional volatility of $x_{d, t}$. Panels (b)(d) plot the analogous loadings on the expected inflation-growth factor $x_{d, t}$, the risk-free-rate factor $x_{f, t}$, and the price-of-risk factor $x_{\lambda, t}$, respectively. 
(a) Real; Level

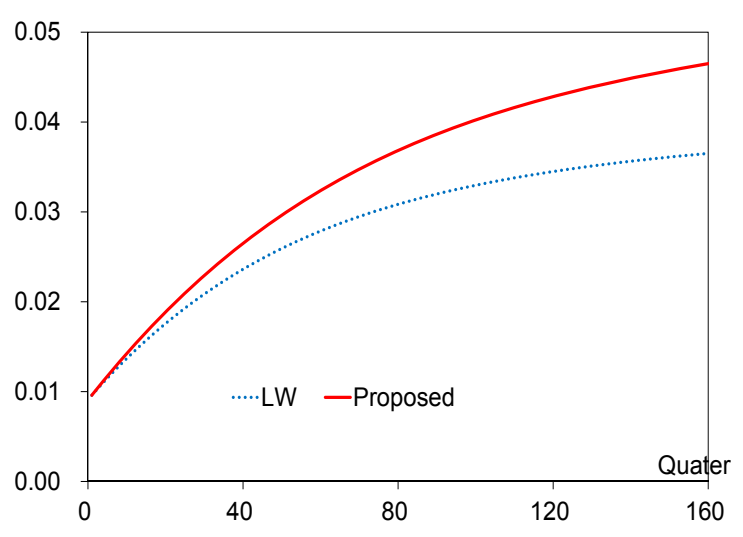

(c) Real; Volatility

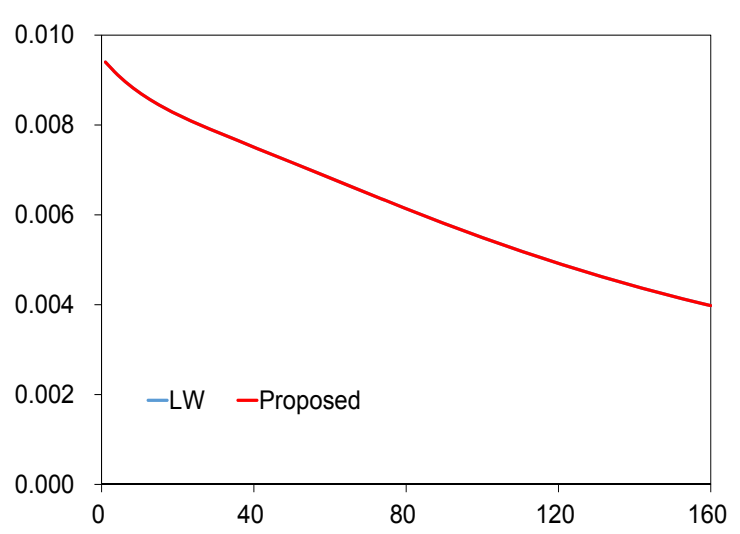

(b) Nominal; Level

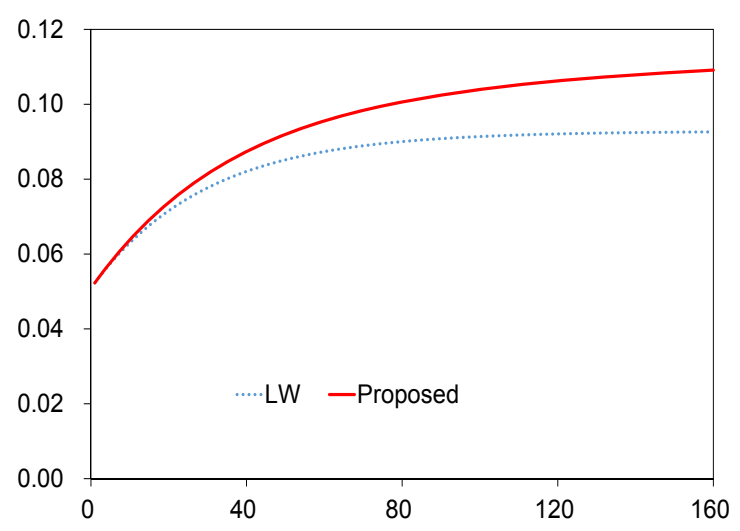

(d) Nominal; Volatility

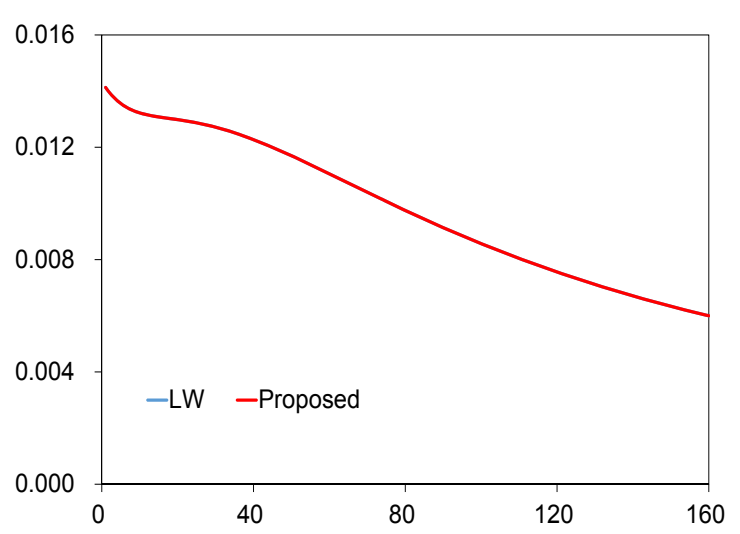

Figure 3: Average term structures of interest rates and volatilities

In Panels (a) and (b), the term structures of $4 E\left[Y_{t, n}^{i}\right](i=\{R, N\})$ (annualized) are plotted against $n$ (quarters), where $Y_{t, n}^{i}$ is the yield to maturity of a zero-coupon bond at time $t$. In Panels $(\mathrm{c})$ and $(\mathrm{d})$, the term structures of $\sqrt{4 \operatorname{var}\left[Y_{t, n}^{i}\right]}(i=\{R, N\})$ (annualized) are plotted. The solid (dotted) line is for the proposed (LW) model. 
(a) Real; $x_{f, t}=0,+2 \mathrm{SD},-2 \mathrm{SD}$

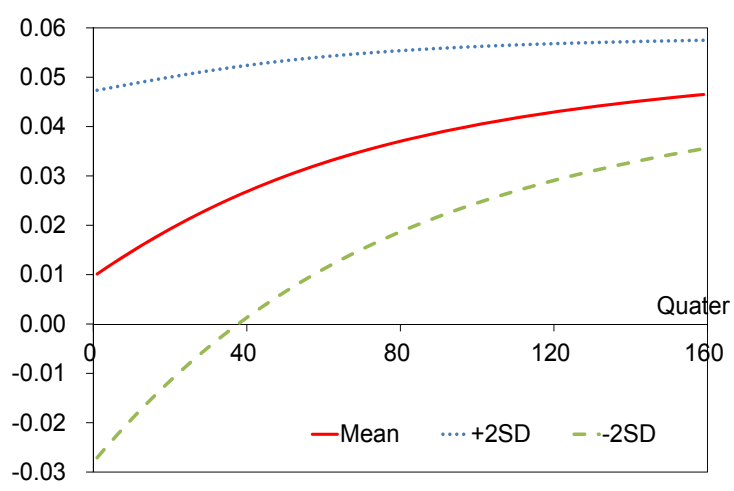

(c) Real; $x_{\lambda, t}=0,+2 \mathrm{SD},-2 \mathrm{SD}$

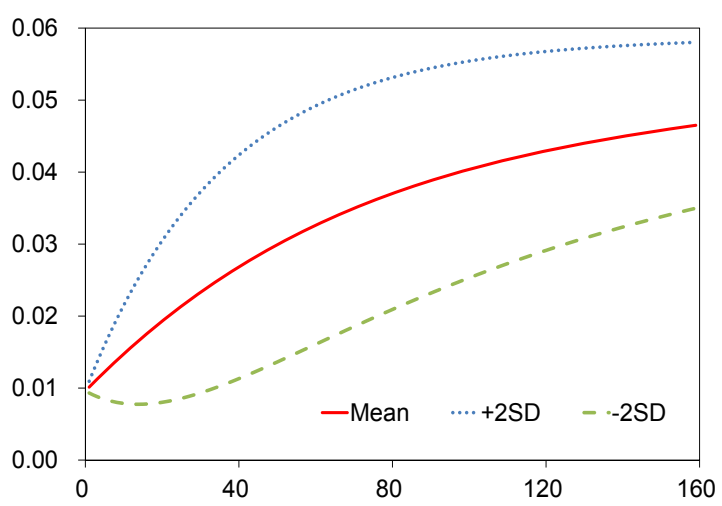

(e) Real; good and bad states

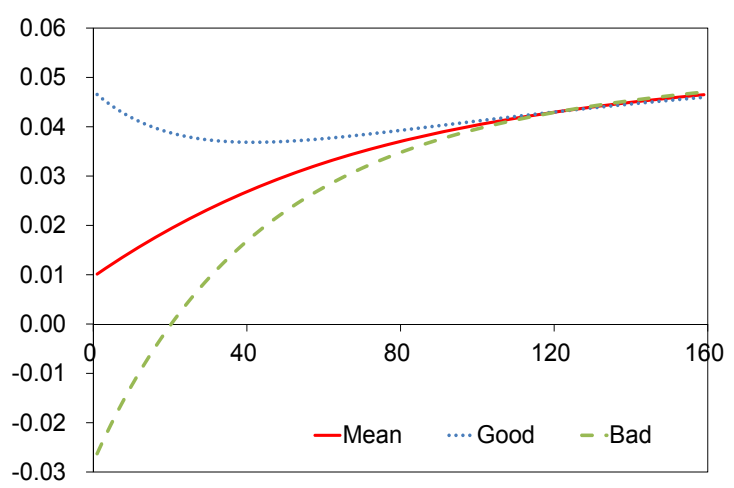

(b) Nominal; $x_{f, t}=0,+2 \mathrm{SD},-2 \mathrm{SD}$

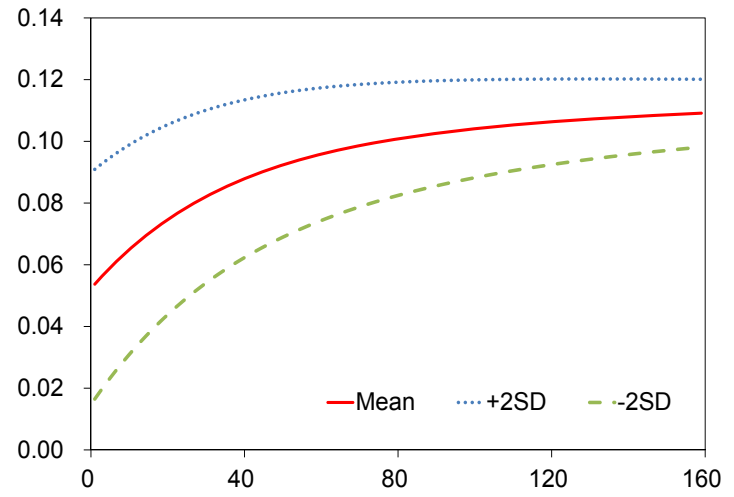

(d) Nominal; $x_{\lambda, t}=0,+2 \mathrm{SD},-2 \mathrm{SD}$

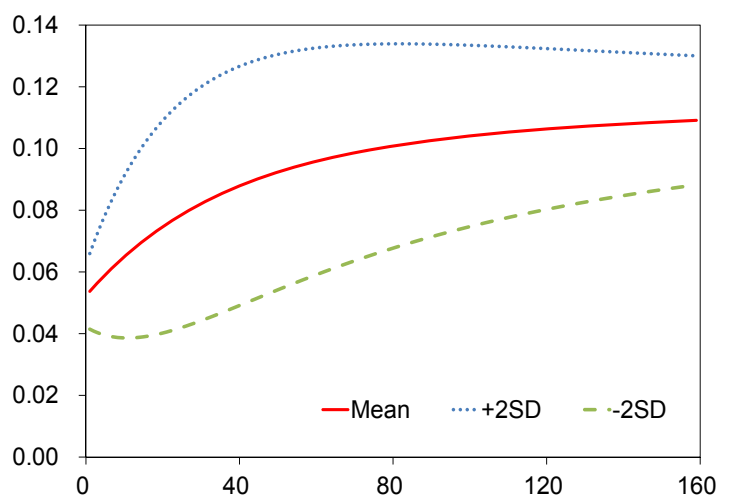

(f) Nominal; good and bad states

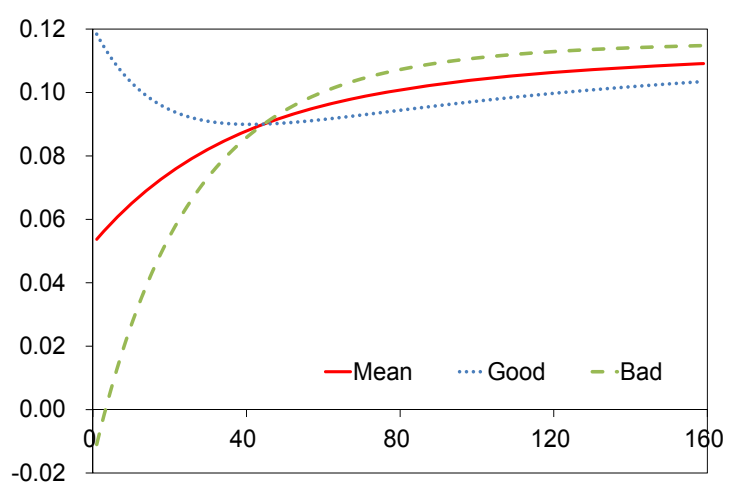

Figure 4: Conditional term structures of interest rates for the proposed model

Panels (a) and (b) plot the term structures when the risk-free-rate factor $x_{f, t}$ is above $(+2 \mathrm{SD})$ or below $(-2 \mathrm{SD})$ two standard deviations from the mean with the other factors fixed at the mean. Panels (c) and (d) plot the term structures when the price-of-risk factor $x_{\lambda, t}$ is above $(+2 \mathrm{SD})$ or below $(-2 \mathrm{SD})$ two standard deviations from the mean with the other factors fixed at the mean. Panels (e) and (f) plot the term structures when the economy is "Good" or "Bad" with a good (bad) state defined as when $x_{\lambda, t}$ is below (above) two standard deviations from the mean and the other factors are above (below) two standard deviations from the mean. 
(a) Risk premiums

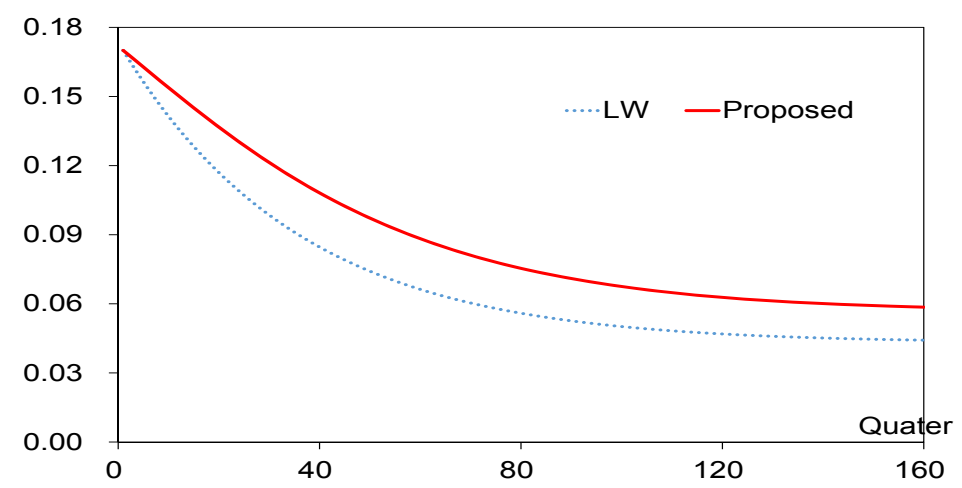

(b) Volatilities

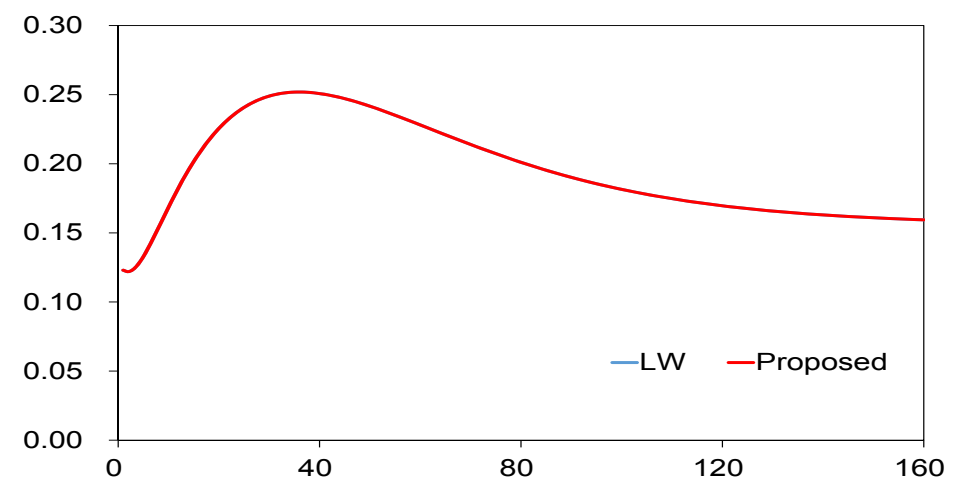

(c) Sharpe ratios

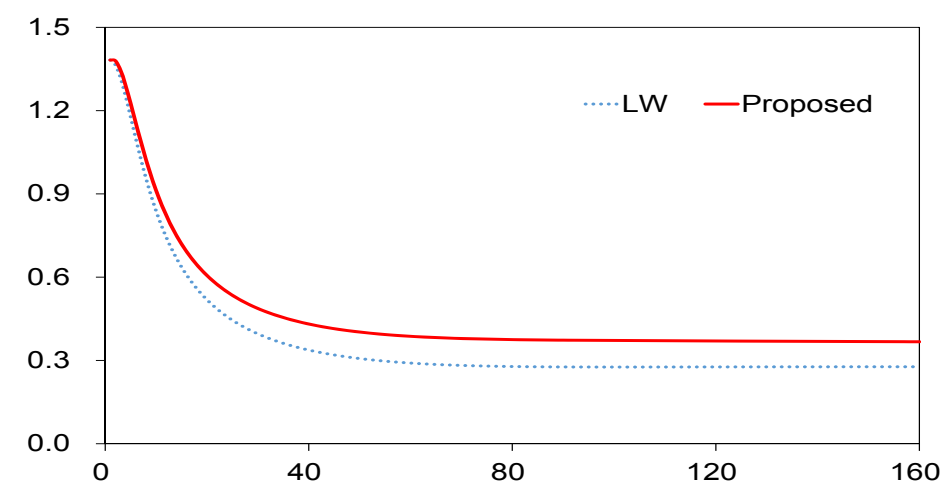

Figure 5: Average term structures of risk premiums, volatilities, and Sharpe ratios for dividend strips (annualized) 
(a) Risk premiums

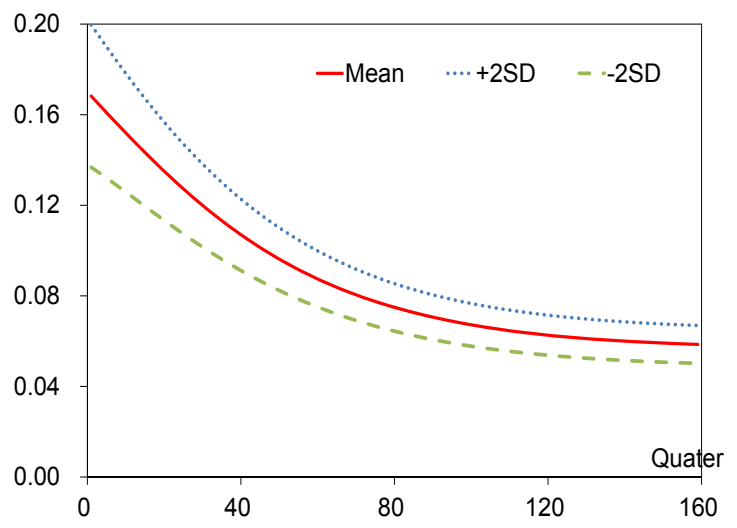

(b) Sharpe ratios

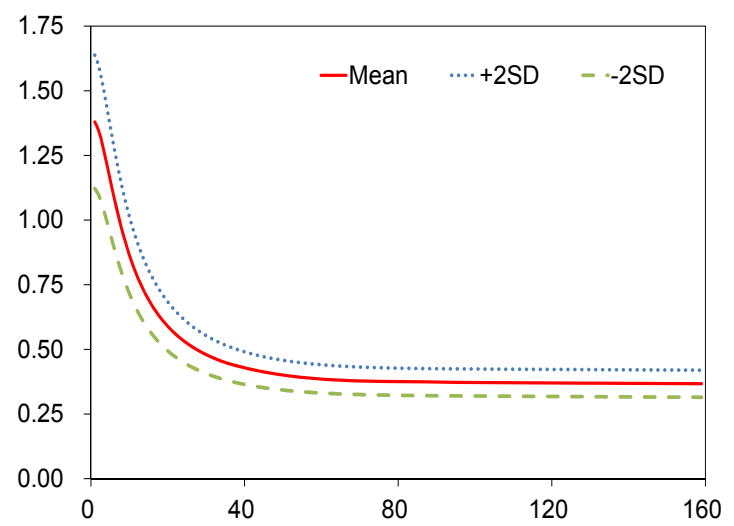

Figure 6: Conditional term structures of dividend risk premiums and Sharpe ratios for the proposed model

The term structures are plotted when the price-of-risk factor $x_{\lambda, t}$ is above $(+2 \mathrm{SD})$ or below $(-2 \mathrm{SD})$ two standard deviations from the mean. 
(a) Real interest rates

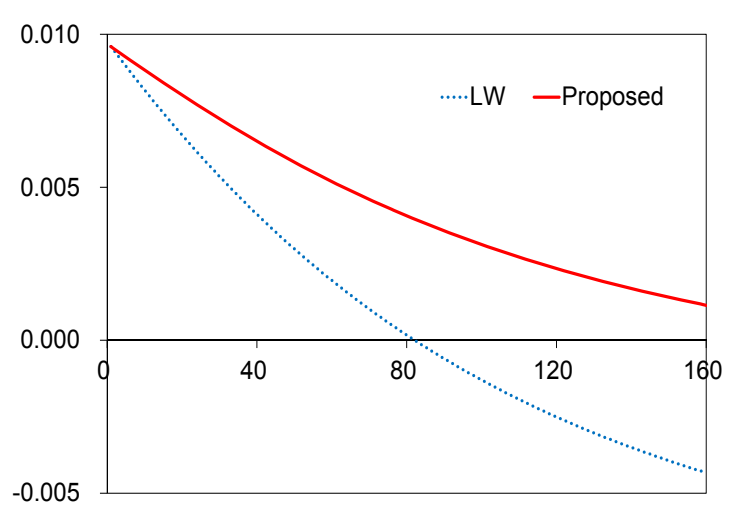

(c) Dividend risk premiums

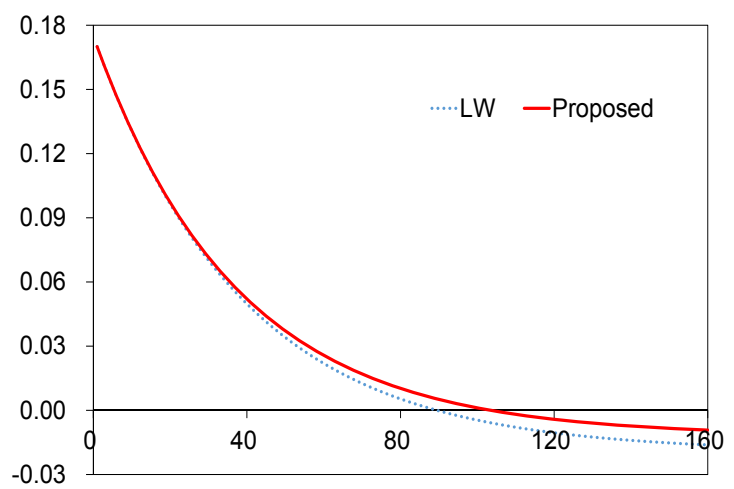

(b) Nominal interest rates

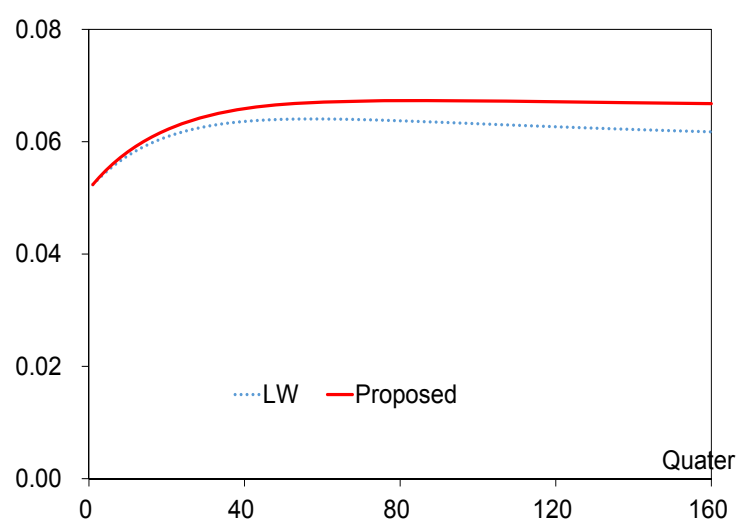

(d) Dividend Sharpe ratios

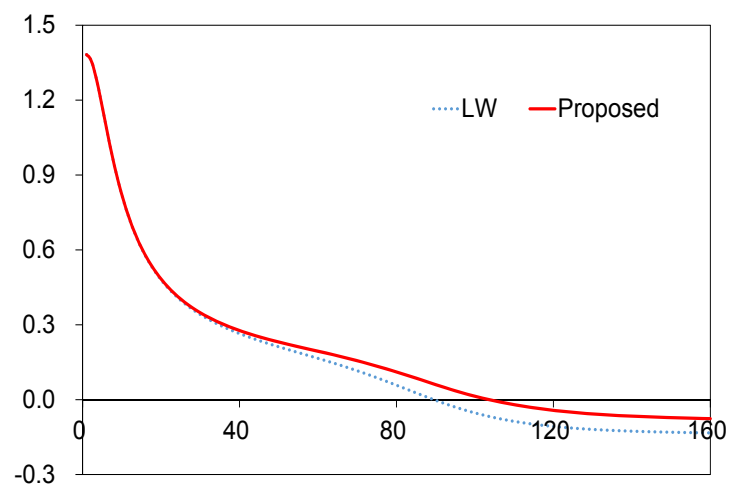

Figure 7: Average term structures of zero-coupon bonds and equities for $\rho_{d x 3}=0.1$ $\rho_{d x 3}$ stands for the correlation between innovations in dividend growth and risk-free-rate factor. It is first changed from -0.3 to 0.1 in the LW model, and then the parameters of the proposed model are re-calibrated in the same procedure as explained in Section 3.2. 
(a) Real; $b_{\gamma 2}=0,90$

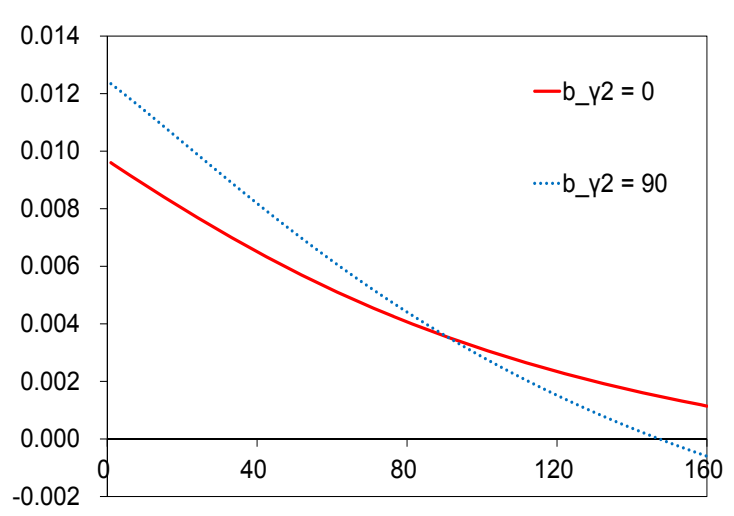

(c) Real; $b_{\beta 2}=0,2$

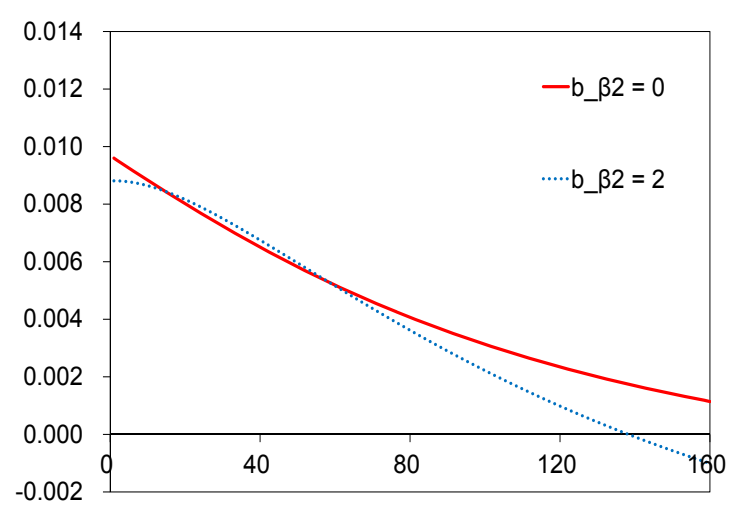

(b) Nominal; $b_{\gamma 2}=0,90$

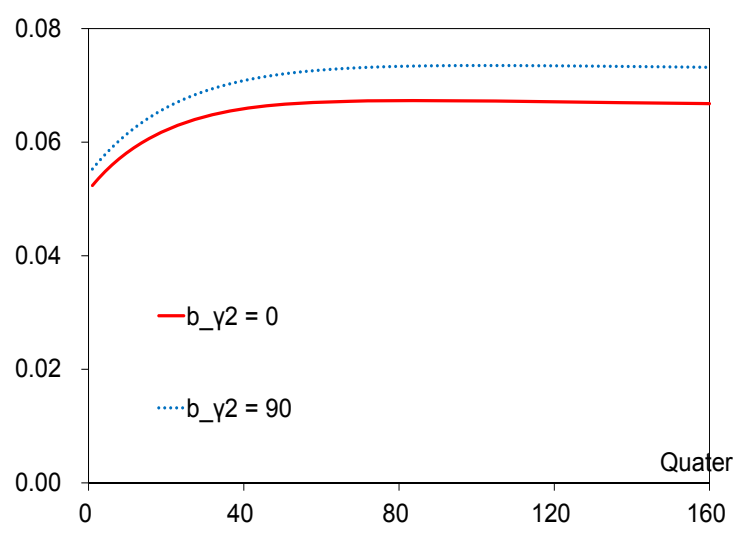

(d) Nominal; $b_{\beta 2}=0,2$

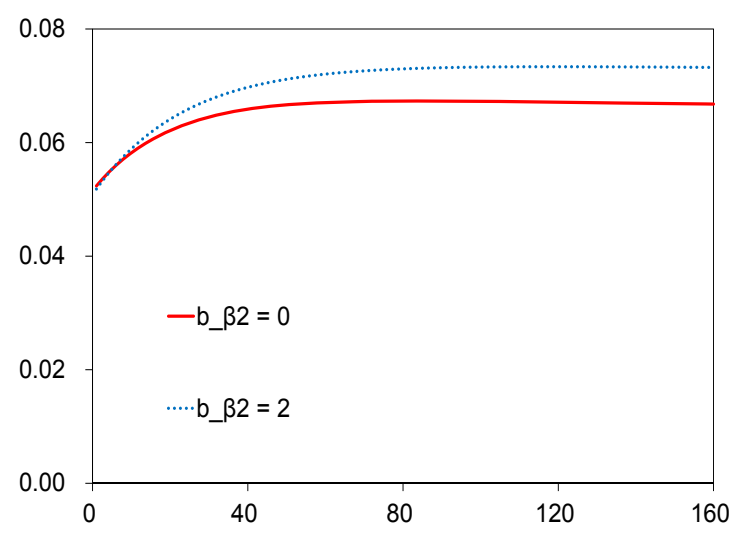

Figure 8: Average term structures of interest rates for $b_{\gamma 2}=90$ or $b_{\beta 2}=2$

$b_{\gamma 2}$ is the coefficient of the expected inflation-growth factor $x_{\pi, t}$ in the risk aversion $\gamma_{t}$ and $b_{\beta 2}$ is the analogous coefficient in the $\log$ subjective discount rate $\ln \left(1-\beta_{t}\right)$. These coefficients, originally set at zero, are changed as indicated above while the other parameters are held fixed at the values presented in Table 5. In each panel, the plot labeled as $b_{\gamma 2}=0$ or $b_{\beta 2}=0$ is the same as shown in Figure 7(a) (real) or Figure 7(b) (nominal). 
(a) Risk aversion $\gamma_{t}$

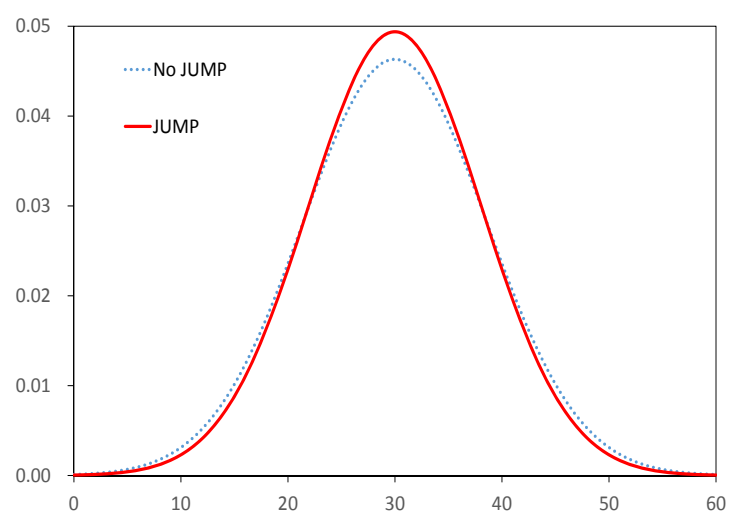

(b) Subjective discount factor $\beta_{t}$

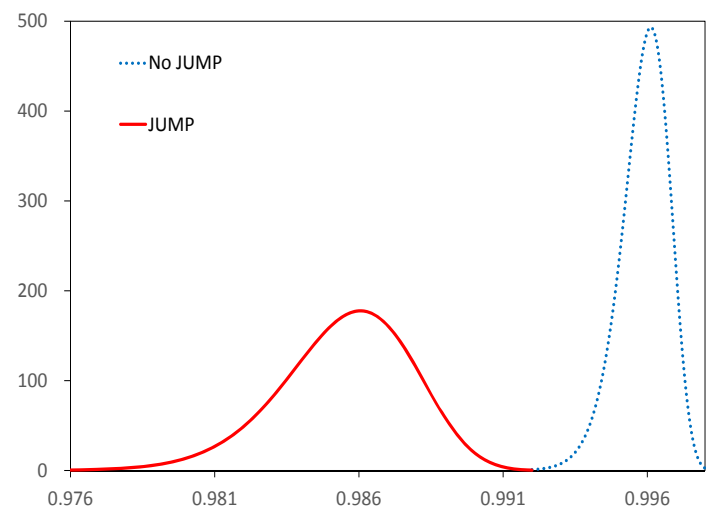

Figure 9: Unconditional distributions of state-dependent preferences with and without jumps when the mean and volatility of $x_{\lambda, t}$ are reduced

The distributions are drawn at parameter values given implicitly in Table 6 . The risk-aversion coefficient is specified as $\gamma_{t}=\mu_{\gamma}+b_{\gamma}^{\prime} x_{t}$ and the subjective discount factor as $\beta_{t}=1-\exp \left\{\mu_{\beta}+b_{\beta}^{\prime} x_{t}\right\}$, where $x_{t}$ is a Gaussian state vector. 
(a) Real; Level

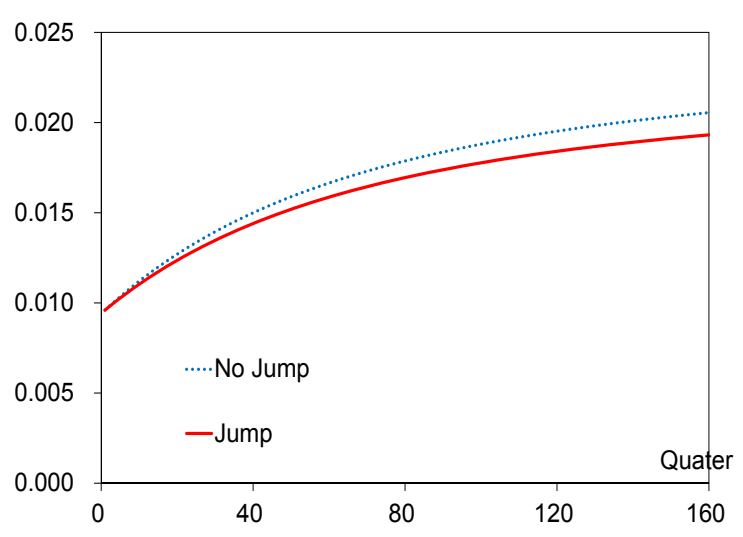

(c) Real; Volatility

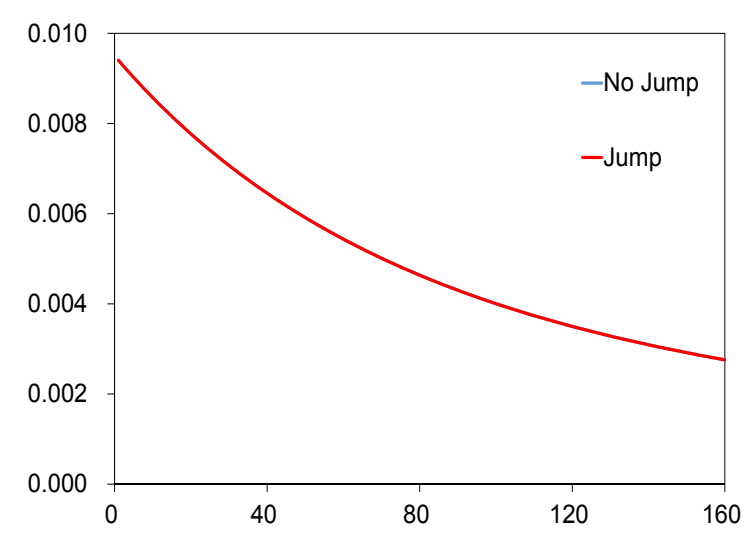

(b) Nominal; Level

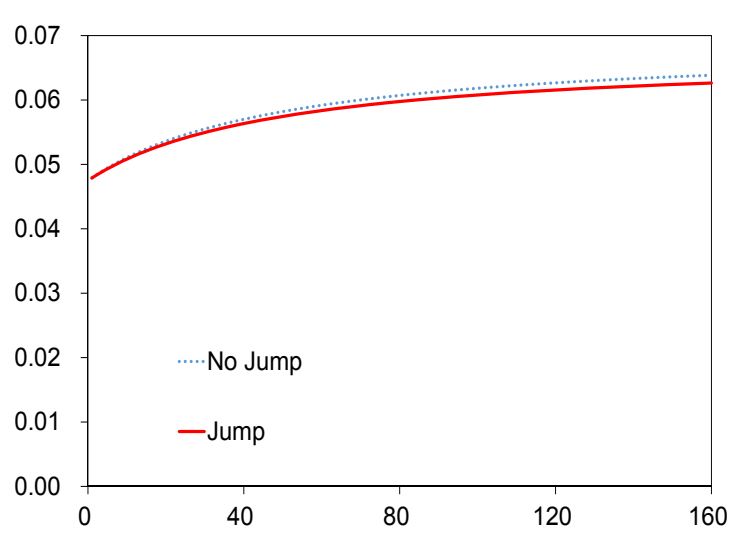

(d) Nominal; Volatility

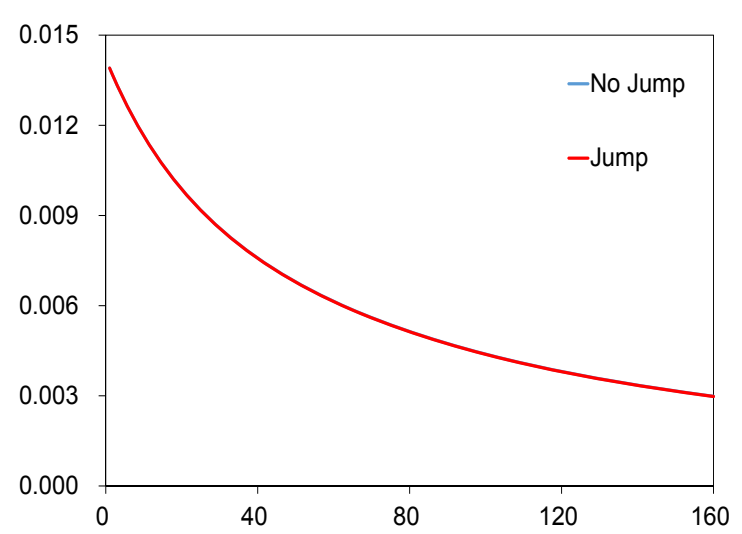

Figure 10: Average term structures of interest rates and volatilities with and without jumps when the mean and volatility of $x_{\lambda, t}$ are reduced

In panels (a) and (b), the term structures of $4 E\left[Y_{t, n}^{i}\right](i=\{R, N\})$ (annualized) are plotted against maturity $n$ (quarters), where $Y_{t, n}^{i}$ is the yield to maturity of a zero-coupon bond at time $t$. In panels $(\mathrm{c})$ and $(\mathrm{d})$, the term structures of $\sqrt{4 \operatorname{var}\left[Y_{t, n}^{i}\right]}(i=\{R, N\})$ (annualized) are plotted. They are drawn at parameter values given implicitly in Table 6 . 
(a) Risk premiums

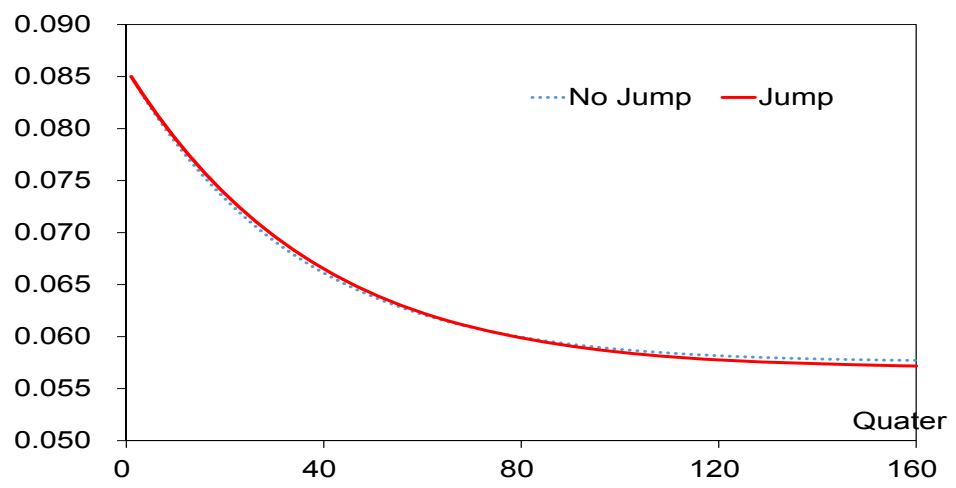

(b) Volatilities

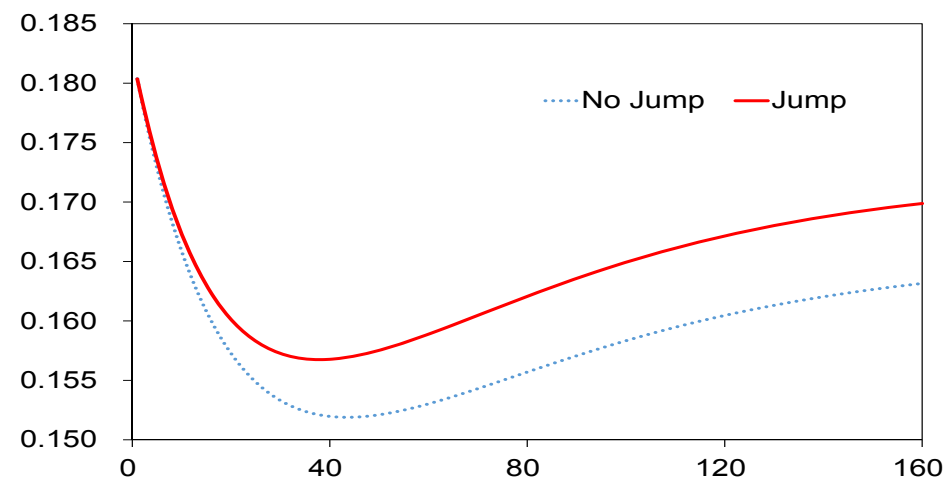

(c) Sharpe ratios

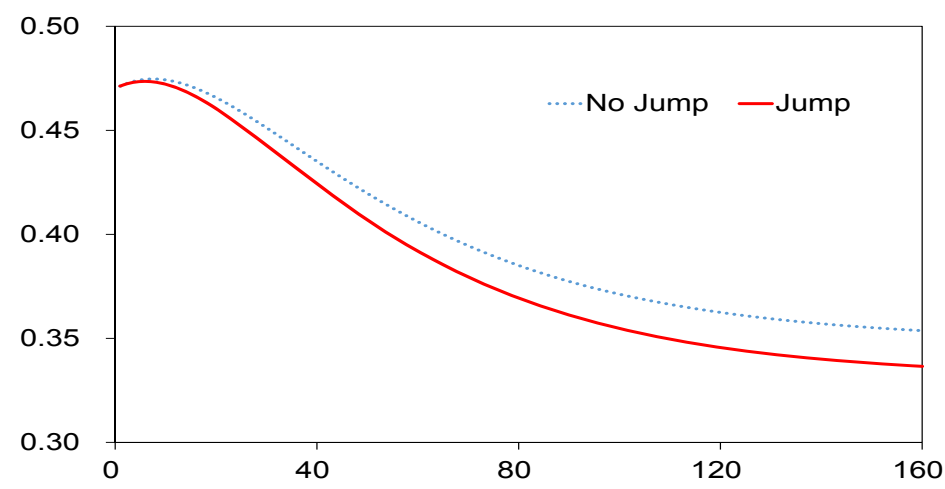

Figure 11: Average term structures of risk premiums, volatilities, and Sharpe ratios for dividend strips (annualized) with and without jumps when the mean and volatility of $x_{\lambda, t}$ are reduced 